\title{
BISECTOR AND ZERO-MACROSPIN CO-ROTATIONAL SYSTEMS FOR SHELL ELEMENTS
}

\author{
B.A. Izzuddin ${ }^{1}$ and Y. Liang ${ }^{2}$
}

\begin{abstract}
A principal issue in any co-rotational approach for large displacement analysis of plates and shells is associated with the specific choice of the local reference system in relation to the current deformed element configuration. Previous approaches utilised local co-rotational systems which are invariant to nodal ordering, a characteristic that is deemed desirable on several fronts; however, the associated definitions of the local reference system suffered from a range of shortcomings, including undue complexity, dependence on the local element formulation, and possibly an asymmetric tangent stiffness matrix. In this paper, new definitions of the local co-rotational system are proposed for quadrilateral and triangular shell elements, which achieve the invariance characteristic to the nodal ordering in a relatively simple manner and address the aforementioned shortcomings. The proposed definitions utilise only the nodal coordinates in the deformed configuration, where two alternative definitions, namely bisector and zero-macrospin definitions, are presented for each of quadrilateral and triangular finite elements. In each case, the co-rotational transformations linking the local and global element entities are presented, highlighting the simplicity of the proposed approach. Several numerical examples are finally presented to demonstrate the effectiveness and relative accuracy of the alternative definitions proposed for the local corotational system.
\end{abstract}

Keywords: Large displacements; geometric nonlinearity; co-rotational framework; finite elements; plates; shells.

\footnotetext{
${ }^{1}$ Professor of Computational Structural Mechanics, Department of Civil and Environmental Engineering, Imperial College London, SW7 2AZ, (Corresponding author, b.izzuddin@imperial.ac.uk).

${ }^{2}$ Research student, Department of Civil and Environmental Engineering, Imperial College London SW7 $2 A Z$.
} 


\section{INTRODUCTION}

In recent years, the geometrically nonlinear analysis of plates and shells has drawn increasing interest in various fields of engineering, including for example in civil engineering the assessment of building structures subject to extreme loading. In formulating large displacement finite elements for small strain problems, the relationship between the strain and displacement fields is highly nonlinear and complex if the displacement fields are referred to a fixed coordinate system, where the nonlinear strain terms arise mainly from the element rigid body rotations. The co-rotational approach, which decomposes the element motion into rigid body and strain-inducing parts via the use of a local co-rotational system, offers exceptional benefits for large displacement structural analysis problems with deformations of the bending type, particularly when accounting for arbitrarily large rigid body rotations. By choosing an element-specific co-rotational reference system which follows the element current deformed configuration, rigid body rotations of the element are removed, and low-order, even linear, relationships between the strain and local displacement fields may be employed. Therefore, the co-rotational approach shifts the focus of large displacement modelling from relating the strain and displacement fields to establishing transformations between local co-rotational and global nodal entities, hence effectively decoupling the large displacement issues from the local element discretization of the continuum response. In this respect, the co-rotational approach has the potential to be applied as an element independent procedure [1,2], upgrading linear local element formulations of different sophistication to geometric nonlinear analysis with relative ease.

A principal issue in any co-rotational approach is associated with the specific choice of the local reference system in relation to the current deformed element configuration. Depending on the specific definition of the local co-rotational system, the resulting co-rotational approach may be element independent but restricted to elements of a specific shape and order, or it may be more generally applied to elements of a particular shape regardless of order, which is the aim of the present work.

Rankin and Brogan [1] defined two co-rotational systems for 3-noded triangular and 4noded quadrilateral elements, respectively, where in both cases one of the local system axes 
was effectively aligned to one of the element edges. These definitions of the co-rotational system were used by Li et al. [3,4] in 6-noded triangular elements for problems involving geometric and material nonlinearity, and by Jiang and Chernuka [5] in a 4-noded quadrilateral element for large displacement analysis. Norachan et al. [6] employed a corotational system for an 8-noded degenerated shell element, utilising the enhanced assumed strain and advanced natural strain concepts for the treatment of locking [7,8], where one of the local system axes was aligned with one of the planar covariant base vectors. Alves de Sousa et al. [9] also considered a co-rotational approach for a degenerated shell element, though the co-rotational transformations were applied at the constitutive integration point level, thus losing the desirable characteristics of element independence and decoupling between the co-rotational transformations and the local element formulation.

Whilst an arbitrary definition that simply requires the local co-rotational system to closely follow the current element configuration, as in the above definitions $[1,6]$, may not significantly affect the large displacement response predictions for small strain problems, this often leads to local system definitions which are not invariant to the specified order of the element nodes. Besides the errors that could arise with such definitions when elements in the same mesh are defined using different nodal ordering, it has also been argued that the invariance characteristic would be desirable for extending the co-rotational approach to large strain problems [2] and for identifying the bifurcation points of perfectly symmetric structures [10].

Towards this end, several approaches were previously proposed to achieve the invariance of the local system to nodal ordering. Kebari and Cassell [11] defined the co-rotational system for a 9-noded quadrilateral shell element by locating the two planar axes symmetrically with respect to the two planar curvilinear coordinates at each integration point. Kim and his colleagues [12-14] employed this definition of the co-rotational system in the formulation of 4-noded and 8-noded monolithic and laminated shell elements for analysis of both elastic and elasto-plastic problems, but the alignment of the planar axes was performed at the element centroid only rather than at all integration points. Crisfield and Moita [2, 15] proposed a co-rotational system that enforced zero local spin at the element centroid using 
polar decomposition, which they employed for 2D/3D continuum elements as well as shell elements. A common feature of these definitions [2, 11-15] is their reliance on the local element displacement fields, which restricts their application in each instance to elements of the same shape and order; in this respect, these definitions are only partially element independent. It is also worth noting that Crisfield and Moita [2] indicated that their approach leads to an asymmetric geometric stiffness matrix, which is obviously undesirable from a computational perspective. In reality, the geometric stiffness matrix, which is directly related to the second derivatives of local with respect to global nodal displacements, cannot be asymmetric when the local co-rotational system is uniquely defined in terms of global nodal displacements. Whilst this may at first cast doubt on the uniqueness of the co-rotational system proposed by Crisfield and Moita [2], it is more credible that their finding of an asymmetric geometric stiffness matrix is incorrect.

There are a few other definitions of the local co-rotational system which not only possess the invariance characteristic to nodal ordering but are also defined in terms of only nodal position variables, thus making them potentially independent of the local element formulation and its order. Rankin [16] defined a co-rotational system by minimising the square of Euclidean norm of nodal local displacements, where the local system orientation was determined through an iterative procedure. This definition was adopted by Eriksson and Pacoste [17] and later refined by Battini and Pacoste [10] for a 3-noded triangular shell element where the need for iteration was overcome with explicit expressions for the orientation of the local system. Importantly, Battini and Pacoste [10] developed similar expressions for the 3-noded triangular element considering also the zero spin definition [2], and they noted that both alternative definitions may be directly used for elements of higher order. Whilst achieving considerable simplification over the previous approaches $[2,16]$, the approach developed by Battini and Pacoste [10] employs two stages, where in the first stage local entities are determined for a local system that follows one of the element edges, and this is then subjected to a spin rotation in a second stage to determine its final orientation. A simpler bisector definition was previously proposed by the first author [18] for 4-noded quadrilateral elements, where the local planar axes are defined as the bisectors of interior 
angles formed by the intersection of the two element diagonals. Not only does this definition of the local co-rotational system achieve nodal invariance as well as independence of the local element formulation and order, but it is also easily and directly determined from global nodal position variables. This co-rotational system was subsequently employed by Li et al. [19-21] in the formulation of a 9-noded shell element applied to multi-layered shell problems and elasto-plastic analysis.

In this paper, new definitions of the local co-rotational system are proposed for quadrilateral and triangular shell elements, which achieve the invariance characteristic to nodal ordering in a relatively simple manner, result in a symmetric tangent stiffness matrix, and are independent of the local element formulation and order. This paper elaborates the bisector definition to deal with triangular elements, and proposes an alternative and equally simple definition based on a zero-macrospin criterion that is also applicable to triangular as well as quadrilateral elements. The proposed bisector and zero-macrospin definitions utilise only nodal coordinates in the deformed configuration, where the co-rotational transformations linking the local and global element response are presented. The new definitions are equally applicable to plates and curved shells, and are thus considered in this paper with flat as well as curved quadrilateral and triangular shell elements utilising the Reissner-Mindlin hypothesis.

The paper proceeds with a discussion on the importance of the invariance characteristic to nodal ordering for the co-rotational approach. Subsequently, the proposed bisector and zeromacrospin definitions of the co-rotational system are respectively presented. The application of the two co-rotational approaches to triangular and quadrilateral shell elements is presented along with an outline of the element formulations in the local system. Several numerical examples are finally provided to demonstrate the effectiveness and relative accuracy of the two proposed co-rotational system definitions.

\section{INVARIANCE CHARACTERISTIC OF CO-ROTATIONAL SYSTEMS}

The simplest definition of a local co-rotational system is based on orienting the local $\mathrm{x}$-axis parallel to the element edge 1-2, with the local z-axis taken normal to edge 1-2 and its 
neighbouring edge, as depicted in Figure 1 for a triangular element. The local element displacement parameters, which exclude rigid body rotations, are then obtained by aligning the local element co-rotational systems for the initial undeformed and current deformed configurations (see 'Local reference system 1' in Figure 1). If the element node numbers are specified in a different order, as labelled in Figure 1 with enclosing parentheses (...), different local displacement parameters are obtained (see 'Local reference system 2' in Figure 1). For this specific element geometry and deformed configuration, it appears that the first local system is associated with reduced local element spin, in the sense that the orientation of the internal line connecting node 3 to the element centroid maintains an almost identical orientation to its initial direction, while the other two internal lines associated with nodes 1 and 2 rotate respectively clockwise and counter-clockwise from their initial directions. However, for the second local system, a resultant counter-clockwise spin of the current configuration relative to the initial configuration is observed, which indicates that rigid body rotations have not been full eliminated from the local deformations. While negligible element spin is a requirement for the viability of a linear local element formulation, this is indeed the case for small strain problems where the effective local element response is identical for the two alternative sets of nodal numbering. The discrepancy between the global element responses with these two sets of nodal numbering does not then arise because of discrepancies in the effective local response but due to differences in the relative orientation of the local co-rotational system in the deformed configuration $\left(\mathbf{c}_{\mathrm{x}}, \mathbf{c}_{\mathrm{y}}, \mathbf{c}_{\mathrm{z}}\right)$ with respect to the initial configuration $\left(\mathbf{c}_{\mathrm{x}}^{\mathrm{o}}, \mathbf{c}_{\mathrm{y}}^{\mathrm{o}}, \mathbf{c}_{\mathrm{z}}^{\mathrm{o}}\right)$, though this difference also reduces with smaller strains.

It is perhaps this observation that led previous researchers to diminish the general importance of invariance to nodal numbering for the co-rotational approach in the context of small strain problems, citing its significance more for extensions to large strain problems [2] and for certain stability problems where symmetry should lead to a loss of stability by bifurcation [10]. While the importance of nodal invariance for such situations is not disputed, it is propounded in this work that there is also a class of small strain problem for which nodal invariance is particularly important, and that is $2 \mathrm{D}$ problems involving out-of-plane curvature of thin plates and shells. 
To illustrate this point, consider two scenarios: i) a rectangular plate of depth D subject to planar curvature (Figure 2.a), and ii) the same plate with thickness t subject to out-of-plane twisting curvature (Figure 2.b), where in both cases the strains are varying linearly over the depth/thickness with extreme fibre strains of $\pm \varepsilon$. Considering quadrilateral elements of size $h$, it can be easily shown that the difference in relative orientation of the local co-rotational system with respect to the initial configuration considering the two noted sets of nodal ordering are as follows:

$$
\Delta \theta= \begin{cases}2\left(\frac{\mathrm{h}}{\mathrm{D}}\right) \varepsilon & \text { planar curvature } \\ 2\left(\frac{\mathrm{h}}{\mathrm{t}}\right) \varepsilon & \text { twisting curvature }\end{cases}
$$

Clearly, for the case of planar curvature, $\mathrm{h}$ is typically a small fraction of $\mathrm{D}$, hence $\Delta \theta$ is also a small fraction of an already small strain $\varepsilon$. However, for the case out-of-plane curvature of a thin plate, $\mathrm{t}$ is typically a very small fraction of $\mathrm{h}$, hence $\Delta \theta$ can be a large multiple of $\varepsilon$. While in both cases, the co-rotational approach is guaranteed to converge with mesh refinement regardless of nodal ordering, since $\Delta \theta$ reduces with $\mathrm{h}$, nodal invariance has important implications on solution accuracy for relatively coarse meshes and on the achievement of monotonic convergence regardless of nodal ordering between successive refinements, as illustrated in subsequent numerical examples. Besides, achieving nodal invariance for the co-rotational approach typically brings the beneficial effect of reducing spin at the local element level, which improves the accuracy of a local linear formulation for large displacement analysis.

The optimal orientation of the local reference system corresponds to a zero-spin condition [2], which cannot be fulfilled at all material points with a single local element system, as required in an element independent co-rotational approach, for general strain variations over the element. Therefore, the aim should generally be to establish a local co-rotational system such that the rotations of infinitesimal segments at different material points within the element domain, from the local undeformed to the deformed configuration, are minimised. 
Within this context, a definition of the local co-rotational system is considered to be effective if it has the following characteristics:

(a) simple to establish,

(b) achieves nodal invariance,

(c) reduces the spin of the element, and

(d) is uniquely defined so as to provide a symmetric element tangent stiffness matrix.

In this paper, two definitions of the local co-rotational system are proposed, namely bisector and zero-macrospin definitions, both of which satisfy the aforementioned requirements, as presented in the following sections.

\section{BISECTOR DEFINITIONS}

Consideration is given hereafter to simple bisector definitions of the local co-rotational system for quadrilateral and triangular elements, which are invariant to nodal ordering, are independent of the local element formulation and order, and provide a symmetric tangent stiffness matrix [18] by virtue of achieving explicit kinematic transformations between the global and local reference systems.

\subsection{Quadrilateral Element}

The local co-rotational system proposed previously [18] for a 4-noded shell element is depicted in Figure 3, where the local $\mathrm{x}$ and $\mathrm{y}$ axes are chosen as the bisectors of the two diagonals of the undeformed element, and rigid body rotations are extracted by orienting these local planar axes so as to also bisect the element diagonals in the current deformed configuration. Clearly, this simple definition automatically satisfies the orthogonality requirement for the two planar axes, and importantly it leads to a relative local orientation of the deformed to the undeformed configuration which is invariant to nodal ordering. On the latter point, it is true that starting from a different node leads to different local axes; however, the eight possible sets of axes represent permutations over three specific orthogonal directions relative to the global system, which always leads to the same global element forces and tangent stiffness matrix regardless of the element nodal ordering. This bisector definition 
implies that the local rotations of the element diagonals, from the undeformed to the deformed configuration, are minimised, as can be observed from the right inset of Figure 3.

With reference to Figure 3, the triad $\left(\mathbf{c}_{\mathrm{x}}, \mathbf{c}_{\mathrm{y}}, \mathbf{c}_{\mathrm{z}}\right)$ defining the current orientation of the local co-rotational system relative to the global system is simply obtained as [18]:

$$
\mathbf{c}_{\mathrm{x}}=\frac{\mathbf{c}_{13}-\mathbf{c}_{24}}{\left|\mathbf{c}_{13}-\mathbf{c}_{24}\right|}, \quad \mathbf{c}_{\mathrm{y}}=\frac{\mathbf{c}_{13}+\mathbf{c}_{24}}{\left|\mathbf{c}_{13}+\mathbf{c}_{24}\right|}, \quad \mathbf{c}_{\mathrm{z}}=\mathbf{c}_{\mathrm{x}} \times \mathbf{c}_{\mathrm{y}}
$$

with:

$$
\mathbf{c}_{\mathrm{ij}}=\frac{\mathbf{v}_{\mathrm{ij}}}{\left|\mathbf{v}_{\mathrm{ij}}\right|}, \quad \mathbf{v}_{\mathrm{ij}}=\mathbf{v}_{\mathrm{ij}}^{\mathrm{o}}+\mathbf{d}_{\mathrm{j}}-\mathbf{d}_{\mathrm{i}}
$$

where $\mathbf{v}_{\mathrm{ij}}^{\mathrm{o}}$ is the vector connecting node $i$ to node $j$ in the initial element configuration, and $\mathbf{d}_{\mathrm{i}}=\left\langle\mathrm{U}_{\mathrm{i}}, \mathrm{V}_{\mathrm{i}}, \mathrm{W}_{\mathrm{i}}\right\rangle^{\mathrm{T}}$ represents the global translational displacements of node $i$.

\subsection{Triangular Element}

A bisector definition of the local co-rotational system for the triangular element becomes slightly more involved than for the quadrilateral element, particularly when the property of invariance to nodal ordering is to be achieved. In this respect, it is proposed that the invariance characteristic can be attained by considering the three internal angles $(\alpha, \beta, \gamma)$, subtended by the three lines connecting the triangle vertices to its centroid $\mathrm{O}$, and aligning the bisectors of the angle that has changed most from the undeformed configuration (say angle $\delta \alpha$ ), as illustrated in the right inset of Figure 4 for alignment of the bisectors of $\alpha$. Clearly, such a definition leads again to a relative local orientation of the deformed to the undeformed configuration which is invariant to nodal ordering, ensuring further that the local rotations of the three centroidal lines, from the undeformed to the deformed configuration, are minimised.

For the case where alignment of the bisector of $\alpha$ governs, the triad $\left(\mathbf{c}_{\mathrm{x}}, \mathbf{c}_{\mathrm{y}}, \mathbf{c}_{\mathrm{z}}\right)$ defining the current orientation can be established as follows:

$$
\mathbf{c}_{\mathrm{x}}=\mathrm{a}_{\mathrm{x}} \mathbf{c}_{\alpha}-\mathrm{a}_{\mathrm{y}} \hat{\mathbf{c}}_{\alpha}, \quad \mathbf{c}_{\mathrm{y}}=\mathrm{a}_{\mathrm{y}} \mathbf{c}_{\alpha}+\mathrm{a}_{\mathrm{x}} \hat{\mathbf{c}}_{\alpha}, \quad \mathbf{c}_{\mathrm{z}}=\frac{\mathbf{v}_{\mathrm{O} 2} \times \mathbf{v}_{\mathrm{O} 3}}{\left|\mathbf{v}_{\mathrm{O} 2} \times \mathbf{v}_{\mathrm{O} 3}\right|}
$$

with: 


$$
\begin{gathered}
\mathbf{c}_{\alpha}=\frac{\mathbf{c}_{\mathrm{O} 2}+\mathbf{c}_{\mathrm{O} 3}}{\left|\mathbf{c}_{\mathrm{O} 2}+\mathbf{c}_{\mathrm{O} 3}\right|}, \quad \hat{\mathbf{c}}_{\alpha}=\mathbf{c}_{\mathrm{z}} \times \mathbf{c}_{\alpha} \\
\mathbf{v}_{\mathrm{Ok}}=\frac{\mathbf{v}_{\mathrm{ik}}+\mathbf{v}_{\mathrm{jk}}}{3}, \quad \mathbf{c}_{\mathrm{Ok}}=\frac{\mathbf{v}_{\mathrm{Ok}}}{\left|\mathbf{v}_{\mathrm{Ok}}\right|} \quad(\mathrm{i} \neq \mathrm{j} \neq \mathrm{k})=1 \rightarrow 3
\end{gathered}
$$

and:

$$
\mathrm{a}_{\mathrm{x}}=\mathbf{c}_{\mathrm{x}}^{\mathrm{o}} \cdot \mathbf{c}_{\alpha}^{\mathrm{o}}, \quad \mathrm{a}_{\mathrm{y}}=\mathbf{c}_{\mathrm{y}}^{\mathrm{o}} \cdot \mathbf{c}_{\alpha}^{\mathrm{o}}
$$

where $\mathbf{v}_{\mathrm{ij}}$ is as given by (3) for the quadrilateral element and superscript $\left(^{\circ}\right)$ refers as before to entities in the initial undeformed configuration. Similar expressions can be employed for the cases in which the alignment of the bisectors of angles $\beta$ or $\gamma$ are governing the orientation of the current local reference system.

It is important to note the alteration of the selected angle during iteration can lead in rare cases to oscillations between two angles, thus causing convergence difficulties, particularly when any two values of $(\delta \alpha, \delta \beta, \delta \gamma)$ are very close. In this respect, it is proposed that the selection of the specific angle to be bisected is fixed during an incremental step according to relative values of $(\delta \alpha, \delta \beta, \delta \gamma)$ at the last known equilibrium configuration.

\section{ZERO-MACROSPIN DEFINITIONS}

As noted before, the optimal orientation of the local reference system provides a rotated undeformed configuration such that the relative spin of the material in the current deformed configuration is zero. An equivalent condition is that the material strains are obtained from the rotated undeformed configuration using 'stretch' operations only, which cannot be fulfilled at all material points within an element that is subject to general strain variations when a single local reference system is used. Although it is possible to satisfy the zero-spin condition at one material point [2], new definitions of the local co-rotational system are proposed here which are based on zero-spin at the macro element level, thus reducing the material spin in an aggregate sense over the element domain. These zero-macrospin definitions of the local co-rotational system maintain all the benefits of the previous bisector definitions, including invariance to nodal ordering, element independence and symmetry of the tangent stiffness matrix. 
To introduce the proposed zero-macrospin definitions of the local co-rotational system, consider a unit square area, defined by orthogonal unit vectors $\mathbf{c}_{\mathrm{x}}=\left\langle\begin{array}{lll}1 & 0\end{array}\right\rangle^{\mathrm{T}}$ and $\mathbf{c}_{\mathrm{y}}=\left\langle\begin{array}{lll}0 & 1\end{array}\right\rangle^{\mathrm{T}}$, which is subjected to a uniform planar 'stretch' operation in any two orthogonal directions leading to transformed vectors $\mathbf{c}_{\mathrm{x}}^{\prime}$ and $\mathbf{c}_{\mathrm{y}}^{\prime}$, as shown in Figure 5. It can be shown that $\mathbf{c}_{\mathrm{x}}$ is always obtained as the normalised sum of $\mathbf{c}_{\mathrm{x}}^{\prime}$ and $\mathbf{c}_{\mathrm{y}}^{\prime \mathrm{n}}$, where $\mathbf{c}_{\mathrm{y}}^{\prime \mathrm{n}}$ is a planar rotation of $\mathbf{c}_{\mathrm{y}}^{\prime}$ by $-\pi / 2$. $\mathbf{c}_{\mathrm{y}}$ is similarly obtained as the normalised sum of $\mathbf{c}_{\mathrm{y}}^{\prime}$ and $\mathbf{c}_{\mathrm{x}}^{\prime \mathrm{n}}$, where $\mathbf{c}_{\mathrm{x}}^{\prime \mathrm{n}}$ is a planar rotation of $\mathbf{c}_{\mathrm{x}}^{\prime}$ by $+\pi / 2$. Accordingly, if $\mathbf{c}_{\mathrm{z}}$ is known, the remaining vectors of the triad are easily obtained from the stretched vectors as:

$$
\mathbf{c}_{\mathrm{x}}=\frac{\mathbf{c}_{\mathrm{x}}^{\prime}+\mathbf{c}_{\mathrm{y}}^{\prime \mathrm{n}}}{\left|\mathbf{c}_{\mathrm{x}}^{\prime}+\mathbf{c}_{\mathrm{y}}^{\prime \mathrm{n}}\right|}, \quad \mathbf{c}_{\mathrm{y}}^{\prime \mathrm{n}}=\mathbf{c}_{\mathrm{y}}^{\prime} \times \mathbf{c}_{\mathrm{z}}, \quad \mathbf{c}_{\mathrm{y}}=\mathbf{c}_{\mathrm{z}} \times \mathbf{c}_{\mathrm{x}}
$$

The proposed inverse transformation may be used in general to satisfy zero-spin at a specific material point, and unlike previous conventional approaches [2] the corresponding local system would be obtained in a simple way without the need for iteration. Notwithstanding, this relatively simple transformation is employed in this work to ensure zero-macrospin at the overall element level, rather than zero-spin at the material level, by attaching the stretched triad vectors to the element vertices, as discussed next for the triangular and quadrilateral elements.

\subsection{Triangular Element}

With reference to Figure 6, in the initial undeformed configuration, the orthogonal unit vectors $\mathbf{c}_{\mathrm{x}}^{\mathrm{o}}$ and $\mathbf{c}_{\mathrm{y}}^{\mathrm{o}}$ are defined such that $\mathbf{c}_{\mathrm{x}}^{\mathrm{o}}$ is aligned with edge 1-2, and these can be expressed in terms of the initial element vectors $\mathbf{v}_{12}^{\mathrm{o}}$ and $\mathbf{v}_{23}^{\mathrm{o}}$ as follows:

$$
\mathbf{c}_{\mathrm{x}}^{\mathrm{o}}=\mathrm{a}_{\mathrm{x} 1} \mathbf{v}_{12}^{\mathrm{o}}+\mathrm{a}_{\mathrm{x} 2} \mathbf{v}_{23}^{\mathrm{o}}, \quad \mathbf{c}_{\mathrm{y}}^{\mathrm{o}}=\mathrm{a}_{\mathrm{y} 1} \mathbf{v}_{12}^{\mathrm{o}}+\mathrm{a}_{\mathrm{y} 2} \mathbf{v}_{23}^{\mathrm{o}}
$$

with the constants $\mathrm{a}_{\mathrm{x} 1}, \mathrm{a}_{\mathrm{x} 2}, \mathrm{a}_{\mathrm{y} 1}$, and $\mathrm{a}_{\mathrm{y} 2}$ determined as:

$$
\begin{gathered}
\mathrm{a}_{\mathrm{x} 1}=\frac{1}{\left|\mathbf{v}_{12}^{\mathrm{o}}\right|}, \quad \mathrm{a}_{\mathrm{x} 2}=0, \quad \mathrm{a}_{\mathrm{y} 1}=-\frac{\mathbf{c}_{12}^{\mathrm{o} T} \mathbf{c}_{23}^{\mathrm{o}}}{\left|\mathbf{v}_{12}^{\mathrm{o}}\right| \sqrt{1-\left(\mathbf{c}_{12}^{\mathrm{o} T} \mathbf{c}_{23}^{\mathrm{o}}\right)^{2}}}, \mathrm{a}_{\mathrm{y} 2}=\frac{1}{\left|\mathbf{v}_{23}^{\mathrm{o}}\right| \sqrt{1-\left(\mathbf{c}_{12}^{\mathrm{o} T} \mathbf{c}_{23}^{\mathrm{o}}\right)^{2}}} \\
\mathbf{c}_{\mathrm{ij}}^{\mathrm{o}}=\frac{\mathbf{v}_{\mathrm{ij}}^{\mathrm{o}}}{\left|\mathbf{v}_{\mathrm{ij}}^{\mathrm{o}}\right|}
\end{gathered}
$$


In the current deformed configuration, the stretched vectors $\mathbf{c}_{\mathrm{x}}^{\prime}$ and $\mathbf{c}_{\mathrm{y}}^{\prime}$ are linked to $\mathbf{v}_{12}$ and $\mathbf{v}_{23}$ by the same geometric relationship:

$$
\mathbf{c}_{\mathrm{x}}^{\prime}=\mathrm{a}_{\mathrm{x} 1} \mathbf{v}_{12}+\mathrm{a}_{\mathrm{x} 2} \mathbf{v}_{23}, \quad \mathbf{c}_{\mathrm{y}}^{\prime}=\mathrm{a}_{\mathrm{y} 1} \mathbf{v}_{12}+\mathrm{a}_{\mathrm{y} 2} \mathbf{v}_{23}
$$

where $\mathbf{v}_{\mathrm{ij}}$ is as given by (3) and the constants $\mathrm{a}_{\mathrm{x} 1}, \mathrm{a}_{\mathrm{x} 2}, \mathrm{a}_{\mathrm{y} 1}$, and $\mathrm{a}_{\mathrm{y} 2}$ are as given by (10).

With the stretched vectors $\mathbf{c}_{\mathrm{x}}^{\prime}$ and $\mathbf{c}_{\mathrm{y}}^{\prime}$ obtained according to (12), the rotated unit vectors $\mathbf{c}_{\mathrm{x}}$ and $\mathbf{c}_{\mathrm{y}}$, defining the current orientation of the local co-rotational system, can now be established from the inverse 'stretch' operation given by (8), taking $\mathbf{c}_{\mathrm{z}}$ as the unit normal vector in the current deformed configuration, which is expressed as:

$$
\mathbf{c}_{\mathrm{z}}=\frac{\mathbf{v}_{12} \times \mathbf{v}_{23}}{\left|\mathbf{v}_{12} \times \mathbf{v}_{23}\right|}
$$

It is noted that the orientation of an undeformed triangle can always be uniquely determined so that a corresponding triangle of any deformed shape may be obtained using a uniform 'stretch' operation. Therefore, the proposed approach based on zero macrospin is invariant to nodal numbering for the triangular element, since the relative orientation of the rotated undeformed and the current deformed configurations is uniquely defined.

\subsection{Quadrilateral Element}

Unlike the triangular shape, it is not possible to obtain an arbitrarily deformed quadrilateral shape using a single uniform 'stretch' operation, regardless of the orientation of the undeformed quadrilateral. This can be easily appreciated from the fact that each of the component triangles in the quadrilateral shape may require a different orientation of the undeformed configuration to obtain the corresponding deformed shape from a 'stretch' operation. Therefore, the application of the zero-macrospin condition to the quadrilateral element could lead to different relative orientations of the current undeformed and deformed configurations, depending on which three nodes are attached to the stretched planar vectors, thus violating invariance to nodal ordering. This potential shortcoming is overcome in this paper by linking the stretched planar vectors to all four nodes via the two diagonals.

Assuming the local $\mathrm{x}$ and $\mathrm{y}$ axes to be the bisectors of the diagonals in the undeformed configuration, as shown in Figure 3, the following relationship is obtained: 


$$
\mathbf{c}_{\mathrm{x}}^{\mathrm{o}} \equiv \frac{\mathbf{c}_{13}^{\mathrm{o}}-\mathbf{c}_{24}^{\mathrm{o}}}{\left|\mathbf{c}_{13}^{\mathrm{o}}-\mathbf{c}_{24}^{\mathrm{o}}\right|}=\mathrm{a}_{\mathrm{x} 1} \mathbf{v}_{13}^{\mathrm{o}}+\mathrm{a}_{\mathrm{x} 2} \mathbf{v}_{24}^{\mathrm{o}}, \quad \mathbf{c}_{\mathrm{y}}^{\mathrm{o}} \equiv \frac{\mathbf{c}_{13}^{\mathrm{o}}+\mathbf{c}_{24}^{\mathrm{o}}}{\left|\mathbf{c}_{13}^{\mathrm{o}}+\mathbf{c}_{24}^{\mathrm{o}}\right|}=\mathrm{a}_{\mathrm{y} 1} \mathbf{v}_{13}^{\mathrm{o}}+\mathrm{a}_{\mathrm{y} 2} \mathbf{v}_{24}^{\mathrm{o}}
$$

with the constants $\mathrm{a}_{\mathrm{x} 1}, \mathrm{a}_{\mathrm{x} 2}, \mathrm{a}_{\mathrm{y} 1}$, and $\mathrm{a}_{\mathrm{y} 2}$ determined as:

$$
\begin{aligned}
& a_{x 1}=\frac{1}{\left|\mathbf{v}_{13}^{\mathrm{o}}\right|\left|\mathbf{c}_{13}^{\mathrm{o}}-\mathbf{c}_{24}^{\mathrm{o}}\right|}, \quad \mathrm{a}_{\mathrm{x} 2}=-\frac{1}{\left|\mathbf{v}_{24}^{\mathrm{o}}\right|\left|\mathbf{c}_{13}^{\mathrm{o}}-\mathbf{c}_{24}^{\mathrm{o}}\right|} \\
& \mathrm{a}_{\mathrm{y} 1}=\frac{1}{\left|\mathbf{v}_{13}^{\mathrm{o}}\right|\left|\mathbf{c}_{13}^{\mathrm{o}}+\mathbf{c}_{24}^{\mathrm{o}}\right|}, \quad \mathrm{a}_{\mathrm{y} 2}=\frac{1}{\left|\mathbf{v}_{24}^{\mathrm{o}}\right|\left|\mathbf{c}_{13}^{\mathrm{o}}+\mathbf{c}_{24}^{\mathrm{o}}\right|}
\end{aligned}
$$

Similar to the triangular element, in the current deformed configuration the stretch vectors can be related to current diagonals $\mathbf{v}_{13}$ and $\mathbf{v}_{24}$ with the same expression:

$$
\mathbf{c}_{\mathrm{x}}^{\prime}=\mathrm{a}_{\mathrm{x} 1} \mathbf{v}_{13}+\mathrm{a}_{\mathrm{x} 2} \mathbf{v}_{24}, \quad \mathbf{c}_{\mathrm{y}}^{\prime}=\mathrm{a}_{\mathrm{y} 1} \mathbf{v}_{13}+\mathrm{a}_{\mathrm{y} 2} \mathbf{v}_{24} .
$$

Again, with the stretched vectors $\mathbf{c}_{\mathrm{x}}^{\prime}$ and $\mathbf{c}_{\mathrm{y}}^{\prime}$ obtained according to (17), the rotated unit vectors $\mathbf{c}_{\mathrm{x}}$ and $\mathbf{c}_{\mathrm{y}}$, defining the current orientation of the local co-rotational system, can now be established from the inverse 'stretch' operation given by (8), taking $\mathbf{c}_{\mathrm{z}}$ as the unit normal vector in the current deformed configuration:

$$
\mathbf{c}_{\mathrm{z}}=\frac{\mathbf{v}_{13} \times \mathbf{v}_{24}}{\left|\mathbf{v}_{13} \times \mathbf{v}_{24}\right|}
$$

Via the use of diagonals in the establishment of the co-rotational coordinates, this zeromacrospin definition of the local co-rotational system for quadrilateral elements provides invariance to nodal ordering. It should be noted that if the deformed quadrilateral configuration represents a uniform 'stretch' of the undeformed configuration, then the proposed zero-macrospin definition ensures the satisfaction of zero-macrospin for all component triangles; otherwise, the latter is typically satisfied only in an average sense.

\section{LOCAL ELEMENT FORMULATIONS}

The proposed definitions of the local co-rotational system are readily applicable to local small strain triangular and quadrilateral shell elements. Flat and curved shell elements are considered in the present work, including in each case triangular and quadrilateral elements, the local kinematics of which are overviewed hereafter. 


\subsection{Flat shell elements}

As is illustrated in Figure 7, two flat shell elements are formulated such that five basic parameters (three translations and two rotations) are utilised at each node in addition to four hierarchic parameters (two normal/tangential planar translations, one normal rotation, and one tangential shear parameter) at each edge. With reference to Figure 7.b, before the application of the edge shear constraints to the element, 6 hierarchic parameters $\left(\widehat{\mathrm{u}}_{\mathrm{i}}, \widehat{\mathrm{v}}_{\mathrm{i}}, \breve{\mathrm{w}}_{1, \mathrm{i}}, \breve{\mathrm{w}}_{2, \mathrm{i}}, \widehat{\theta}_{\mathrm{x}, \mathrm{i}}, \widehat{\theta}_{\mathrm{y}, \mathrm{i}}\right)$ are defined at each edge to allow for quadratic modes for planar displacement $(\mathrm{u}, \mathrm{v})$ and rotation $\left(\theta_{\mathrm{x}}, \theta_{\mathrm{y}}\right)$ fields along with cubic modes for transverse displacement field w, which leads to the following set of parameters:

$$
\begin{gathered}
\tilde{\mathbf{u}}^{\mathrm{e}}=\left\langle\mathbf{u}_{\mathrm{b}}^{\mathrm{T}} \tilde{\mathbf{u}}_{\mathrm{a}}^{\mathrm{T}}\right\rangle^{\mathrm{T}} \\
\mathbf{u}_{\mathrm{b}}=\left\langle\mathrm{u}_{1 \rightarrow \mathrm{n}}: \mathrm{v}_{1 \rightarrow \mathrm{n}}: \mathrm{w}_{1 \rightarrow \mathrm{n}}: \theta_{\mathrm{x}_{1 \rightarrow \mathrm{n}}}: \theta_{\mathrm{y}_{1 \rightarrow \mathrm{n}}}\right\rangle^{\mathrm{T}}, \quad \tilde{\mathbf{u}}_{\mathrm{a}}=\left\langle\widehat{\mathrm{u}}_{1 \rightarrow \mathrm{n}}: \widehat{\mathrm{v}}_{1 \rightarrow \mathrm{n}}: \widehat{\mathrm{w}}_{1 \rightarrow 2 \mathrm{n}}: \hat{\theta}_{\mathrm{x}_{1 \rightarrow \mathrm{n}}}: \hat{\theta}_{\mathrm{y}_{1 \rightarrow \mathrm{n}}}\right\rangle^{\mathrm{T}}
\end{gathered}
$$

where: $\mathbf{u}_{\mathrm{b}}$ is the vector of basic nodal parameters; $\tilde{\mathbf{u}}_{\mathrm{a}}$ contains hierarchic edge parameters before the introduction of edge shear constraints to the element; and $\mathrm{n}$ represents the number

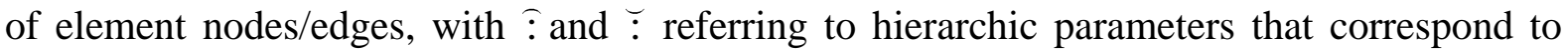
quadratic and cubic hierarchic functions, respectively. Note that the sign convention for displacement fields and hierarchic edge parameters is shown in Figure 8.

The local element geometry and displacement fields for the triangular and quadrilateral elements are interpolated as follows:

$$
\begin{aligned}
& x(\xi, \eta)=\sum_{i=1}^{n} N_{i}^{L} x_{i}, \quad y(\xi, \eta)=\sum_{i=1}^{n} N_{i}^{L} y_{i}, \quad z(\xi, \eta)=\sum_{i=1}^{n} N_{i}^{L} z_{i} \\
& \mathrm{u}(\xi, \eta)=\sum_{\mathrm{i}=1}^{\mathrm{n}} \mathrm{N}_{\mathrm{i}}^{\mathrm{L}} \mathrm{u}_{\mathrm{i}}+\sum_{\mathrm{i}=1}^{\mathrm{n}} \mathrm{N}_{\mathrm{i}}^{\mathrm{Q}}\left(\mathrm{c}_{\mathrm{i}} \widehat{\mathrm{u}}_{\mathrm{i}}-\mathrm{s}_{\mathrm{i}} \widehat{\mathrm{v}}_{\mathrm{i}}\right) \\
& \mathrm{v}(\xi, \eta)=\sum_{\mathrm{i}=1}^{\mathrm{n}} \mathrm{N}_{\mathrm{i}}^{\mathrm{L}} \mathrm{v}_{\mathrm{i}}+\sum_{\mathrm{i}=1}^{\mathrm{n}} \mathrm{N}_{\mathrm{i}}^{\mathrm{Q}}\left(\mathrm{s}_{\mathrm{i}} \widehat{\mathrm{u}}_{\mathrm{i}}+\mathrm{c}_{\mathrm{i}} \widehat{\mathrm{v}}_{\mathrm{i}}\right) \\
& \mathrm{w}(\xi, \eta)=\sum_{\mathrm{i}=1}^{\mathrm{n}} \mathrm{N}_{\mathrm{i}}^{\mathrm{L}} \mathrm{w}_{\mathrm{i}}+\sum_{\mathrm{i}=1}^{\mathrm{n}} \mathrm{N}_{\mathrm{i}}^{\mathrm{C}_{1}} \breve{\mathrm{w}}_{1, \mathrm{i}}+\sum_{\mathrm{i}=1}^{\mathrm{n}} \mathrm{N}_{\mathrm{i}}^{\mathrm{C}_{2}} \breve{\mathrm{w}}_{2, \mathrm{i}} \\
& \theta_{x}(\xi, \eta)=\sum_{i=1}^{n} N_{i}^{\mathrm{L}} \theta_{x, i}+\sum_{i=1}^{n} N_{i}^{Q}\left(c_{i} \widehat{\theta}_{x, i}-s_{i} \widehat{\theta}_{y, i}\right)
\end{aligned}
$$




$$
\theta_{\mathrm{y}}(\xi, \eta)=\sum_{\mathrm{i}=1}^{\mathrm{n}} \mathrm{N}_{\mathrm{i}}^{\mathrm{L}} \theta_{\mathrm{y}, \mathrm{i}}+\sum_{\mathrm{i}=1}^{\mathrm{n}} \mathrm{N}_{\mathrm{i}}^{\mathrm{Q}}\left(\mathrm{s}_{\mathrm{i}} \widehat{\theta}_{\mathrm{x}, \mathrm{i}}+\mathrm{c}_{\mathrm{i}} \widehat{\theta}_{\mathrm{y}, \mathrm{i}}\right)
$$

in which: $\mathrm{N}_{\mathrm{i}}^{\mathrm{L}}, \mathrm{N}_{\mathrm{i}}^{\mathrm{Q}}$ and $\mathrm{N}_{\mathrm{i}}^{\mathrm{C}}$ represent linear (bilinear for quadrilateral element), quadratic and cubic hierarchic functions of $(\xi, \eta)$, respectively; $c_{i}$ and $s_{i}$ represent the direction cosines of the local axis along edge $i-i_{+}$in relation to the local element $\mathrm{x}$-axis; and $\mathrm{n}=3$ for the triangular element while $n=4$ for the quadrilaterial element.

For the 3-noded triangular element, the shape functions are expressed in terms of area coordinates $\left(\mathrm{L}_{1}, \mathrm{~L}_{2}, \mathrm{~L}_{3}\right)$ as follows:

$$
\begin{gathered}
\mathrm{N}_{\mathrm{i}}^{\mathrm{L}}=\mathrm{L}_{\mathrm{i}}, \quad \mathrm{N}_{\mathrm{i}}^{\mathrm{Q}}=4 \mathrm{~L}_{\mathrm{i}} \mathrm{L}_{\mathrm{i}_{+}} \\
\mathrm{N}_{\mathrm{i}}^{\mathrm{C}_{1}}=\frac{9}{2} \mathrm{~L}_{\mathrm{i}} \mathrm{L}_{\mathrm{i}_{+}}\left(2 \mathrm{~L}_{\mathrm{i}}-\mathrm{L}_{\mathrm{i}_{+}}\right), \quad \mathrm{N}_{\mathrm{i}}^{\mathrm{C}_{2}}=\frac{9}{2} \mathrm{~L}_{\mathrm{i}} \mathrm{L}_{\mathrm{i}_{+}}\left(2 \mathrm{~L}_{\mathrm{i}_{+}}-\mathrm{L}_{\mathrm{i}}\right)
\end{gathered}
$$

in which $\mathrm{i}=1 \rightarrow \mathrm{n}$, and $\mathrm{i}_{+}=\bmod (\mathrm{i}, \mathrm{n})+1$. The shape functions can then be expressed in terms of natural coordinates $(\xi, \eta)$ by setting $\mathrm{L}_{1}=1-\xi-\eta, \mathrm{L}_{2}=\xi$, and $\mathrm{L}_{3}=\eta$.

For the 4-noded quadrilateral element, the shape functions are expressed in terms of natural coordinates $(\xi, \eta)$ as follows:

$$
\begin{gathered}
\mathrm{N}_{\mathrm{i}}^{\mathrm{L}}=\frac{1}{4}\left(1-\chi_{\mathrm{i}}\right)\left(1-\chi_{\mathrm{i}_{+}}\right), \quad \mathrm{N}_{\mathrm{i}}^{\mathrm{Q}}=\frac{1}{2}\left(1-\chi_{\mathrm{i}}^{2}\right)\left(1-\chi_{\mathrm{i}_{+}}\right) \\
\mathrm{N}_{\mathrm{i}}^{\mathrm{C}_{1}}=\frac{9}{32}\left(1-\chi_{\mathrm{i}}^{2}\right)\left(1-3 \chi_{\mathrm{i}}\right)\left(1-\chi_{\mathrm{i}_{+}}\right), \quad \mathrm{N}_{\mathrm{i}}^{\mathrm{C}_{2}}=\frac{9}{32}\left(1-\chi_{\mathrm{i}}^{2}\right)\left(1+3 \chi_{\mathrm{i}}\right)\left(1-\chi_{\mathrm{i}_{+}}\right)
\end{gathered}
$$

in which $\mathrm{i}=1 \rightarrow \mathrm{n}, \chi_{1}=\xi, \chi_{2}=\eta, \chi_{3}=-\xi$ and $\chi_{4}=-\eta$.

The tangential shear strain along edge $i-i_{+}, \gamma_{\mathrm{x}, \mathrm{i}}$, can be obtained as a quadratic field in terms of the basic and hierarchic edge parameters:

$$
\begin{aligned}
\gamma_{x, i}\left(\rho_{\mathrm{i}}\right)= & -\mathrm{s}_{\mathrm{i}} \theta_{\mathrm{y}, \mathrm{i}}-\mathrm{c}_{\mathrm{i}} \theta_{\mathrm{x}, \mathrm{i}}+\frac{\mathrm{w}_{\mathrm{i}_{+}}-\mathrm{w}_{\mathrm{i}}}{\mathrm{l}_{\mathrm{i}}}-\frac{9}{2 \mathrm{l}_{\mathrm{i}}}\left(\breve{\mathrm{w}}_{2, \mathrm{i}}-2 \breve{\mathrm{w}}_{1, \mathrm{i}}\right)- \\
& \rho_{\mathrm{i}}\left[\mathrm{c}_{\mathrm{i}}\left(\theta_{\mathrm{x}, \mathrm{i}_{+}}-\theta_{\mathrm{x}, \mathrm{i}}\right)+\mathrm{s}_{\mathrm{i}}\left(\theta_{\mathrm{y}, \mathrm{i}_{+}}-\theta_{\mathrm{y}, \mathrm{i}}\right)+\frac{9}{\mathrm{l}_{\mathrm{i}}}\left(4 \breve{\mathrm{w}}_{2, \mathrm{i}}-5 \breve{\mathrm{w}}_{1, \mathrm{i}}\right)+4 \widehat{\theta}_{\mathrm{x}, \mathrm{i}}\right]- \\
& \rho_{\mathrm{i}}^{2}\left[\frac{81}{2 \mathrm{l}_{\mathrm{i}}}\left(\breve{\mathrm{w}}_{2, \mathrm{i}}-\breve{\mathrm{w}}_{1, \mathrm{i}}\right)-4 \widehat{\theta}_{\mathrm{x}, \mathrm{i}}\right] \quad(\mathrm{i}=1 \rightarrow \mathrm{n})
\end{aligned}
$$

where $1_{\mathrm{i}}$ is the length of edge $i-i_{+} ; \rho_{\mathrm{i}}$ is the fractional distance along edge $i-i_{+}$from node $i$, which is 0 at node $i$ and 1 at node $i_{+}$. 
Constraining this edge tangential strain to a constant value $\widehat{\gamma}_{\mathrm{x}, \mathrm{i}}$, allows the hierarchic parameters $\left(\widehat{\theta}_{\mathrm{x}, \mathrm{i}}, \breve{\mathrm{w}}_{1, \mathrm{i}}, \breve{\mathrm{w}}_{2, \mathrm{i}}\right)$ to be obtained in terms of a reduced set of edge parameters $\left(w_{i}, \theta_{x, i}, \theta_{y, i}, w_{j}, \theta_{x, j}, \theta_{y, j}, \widehat{\gamma}_{x, i}\right)$ with the use of (25), as shown in Figure 7.c. The vector of reduced parameters can then be expressed as:

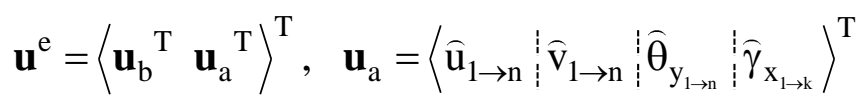

However, for the case of a simple support along the edge $i-i_{+}$a constant $\gamma_{\mathrm{x}, \mathrm{i}}$ cannot be achieved for all combinations of nodal parameters, since the edge must remain straight, and hence the hierarchic parameters $\left(\breve{\mathrm{w}}_{1, \mathrm{i}}, \breve{\mathrm{w}}_{2, \mathrm{i}}\right)$ must be zero. Therefore, for a simply supported edge, $\left(\breve{\mathrm{w}}_{1, \mathrm{i}}, \breve{\mathrm{w}}_{2, \mathrm{i}}, \widehat{\theta}_{\mathrm{x}, \mathrm{i}}\right)$ are taken as zero, which essentially corresponds to a linear variation for $\left(\mathrm{w}, \theta_{\mathrm{x}}, \theta_{\mathrm{y}}\right)$ and $\gamma_{\mathrm{x}, \mathrm{i}}$. Otherwise, the parameters $\left(\breve{\mathrm{w}}_{1, \mathrm{i}}, \breve{\mathrm{w}}_{2, \mathrm{i}}, \widehat{\theta}_{\mathrm{x}, \mathrm{i}}\right)$ are determined from (25) so as to satisfy a constant edge shear strain $\gamma_{\mathrm{x}, \mathrm{i}}$. If a binary variable $\left(\lambda_{\mathrm{i}}\right)$ is used for edge $i-i_{+}$, with values of 0 and 1 indicating the presence and absence of a straight edge constraint, respectively, then the hierarchic edge parameters $\left(\breve{\mathrm{w}}_{1, \mathrm{i}}, \breve{\mathrm{w}}_{2, \mathrm{i}}, \widehat{\theta}_{\mathrm{x}, \mathrm{i}}\right)$ can be expressed in terms of $\left(\mathrm{w}_{1 \rightarrow \mathrm{n}}, \theta_{\mathrm{x}_{1 \rightarrow \mathrm{n}}}, \theta_{\mathrm{y}_{1 \rightarrow \mathrm{n}}}, \widehat{\gamma}_{\mathrm{x}_{1 \rightarrow \mathrm{n}}}\right)$ :

$$
\begin{aligned}
& \left\{\begin{array}{c}
\breve{\mathbf{w}} \\
\hdashline \overline{\boldsymbol{\theta}}_{\mathrm{x}}
\end{array}\right\}=\left[\begin{array}{c:c:c:c}
{ }_{1} \mathbf{C}_{(2 \mathrm{n} \times \mathrm{n})} & \mathbf{C}_{(2 \mathrm{n} \times \mathrm{n})}{ }_{3} \mathbf{C}_{(2 \mathrm{n} \times \mathrm{n})} & { }_{4} \mathbf{C}_{(2 \mathrm{n} \times \mathrm{n})} \\
\hdashline{ }_{5} \mathbf{C}_{(\mathrm{n} \times \mathrm{n})} & { }_{6} \mathbf{C}_{(\mathrm{n} \times \mathrm{n})} & { }_{7} \mathbf{C}_{(\mathrm{n} \times \mathrm{n})} & { }_{8} \mathbf{C}_{(\mathrm{n} \times \mathrm{n})}
\end{array}\right]\left\{\begin{array}{c}
\mathbf{w} \\
\hdashline \boldsymbol{\theta}_{\mathrm{x}} \\
\hdashline \boldsymbol{\theta}_{\mathrm{y}} \\
\hdashline \widehat{\boldsymbol{\gamma}}_{\mathrm{x}}
\end{array}\right\} \\
& { }_{1} \mathbf{C}_{2 \mathrm{i}-1, \mathrm{i}}=-{ }_{1} \mathbf{C}_{2 \mathrm{i}-1, \mathrm{i}_{+}}=-{ }_{1} \mathbf{C}_{2 \mathrm{i}, \mathrm{i}}={ }_{1} \mathbf{C}_{2 \mathrm{i}, \mathrm{i}_{+}}=\frac{2}{27} \lambda_{\mathrm{i}} \\
& { }_{2} \mathbf{C}_{2 \mathrm{i}-1, \mathrm{i}}=-2{ }_{2} \mathbf{C}_{2 \mathrm{i}-1, \mathrm{i}_{+}}=2{ }_{2} \mathbf{C}_{2 \mathrm{i}, \mathrm{i}}=-{ }_{2} \mathbf{C}_{2 \mathrm{i}, \mathrm{i}_{+}}=\frac{4}{27} \lambda_{\mathrm{i}} \mathrm{c}_{\mathrm{i}} \mathrm{l}_{\mathrm{i}} \\
& { }_{3} \mathbf{C}_{2 \mathrm{i}-1, \mathrm{i}}=-2{ }_{3} \mathbf{C}_{2 \mathrm{i}-1, \mathrm{i}_{+}}=2{ }_{3} \mathbf{C}_{2 \mathrm{i}, \mathrm{i}}={ }_{3} \mathbf{C}_{2 \mathrm{i}, \mathrm{i}_{+}}=\frac{4}{27} \lambda_{\mathrm{i}} \mathrm{s}_{\mathrm{i}} \mathrm{l}_{\mathrm{i}} \\
& { }_{4} \mathbf{C}_{2 \mathrm{i}-1, \mathrm{i}}=-{ }_{4} \mathbf{C}_{2 \mathrm{i}, \mathrm{i}}=\frac{2}{27} \lambda_{\mathrm{i}} \mathrm{l}_{\mathrm{i}},{ }_{5} \mathbf{C}_{\mathrm{i}, \mathrm{i}}=-{ }_{5} \mathbf{C}_{\mathrm{i}, \mathrm{i}_{+}}=-\frac{3 \lambda_{\mathrm{i}}}{2 \mathrm{l}_{\mathrm{i}}} \\
& { }_{6} \mathbf{C}_{\mathrm{i}, \mathrm{i}}={ }_{6} \mathbf{C}_{\mathrm{i}, \mathrm{i}_{+}}=-\frac{3}{4} \lambda_{\mathrm{i}} \mathrm{c}_{\mathrm{i}},{ }_{7} \mathbf{C}_{\mathrm{i}, \mathrm{i}}={ }_{7} \mathbf{C}_{\mathrm{i}, \mathrm{i}_{+}}=-\frac{3}{4} \lambda_{\mathrm{i}} \mathrm{s}_{\mathrm{i}},{ }_{8} \mathbf{C}_{\mathrm{i}, \mathrm{i}}=-\frac{3}{2} \lambda_{\mathrm{i}}
\end{aligned}
$$

in which $\mathrm{i}=1 \rightarrow \mathrm{n}$.

Substituting (27) and (28a-e) into (22c-e) leads to: 


$$
\begin{gathered}
\mathrm{w}(\xi, \eta)=\sum_{\mathrm{i}=1}^{\mathrm{n}}\left[\mathrm{N}_{\mathrm{w}, \mathrm{i}}^{\mathrm{w}} \mathrm{w}_{\mathrm{i}}+\mathrm{N}_{\mathrm{w}, \mathrm{i}}^{\theta_{\mathrm{x}}} \theta_{\mathrm{x}, \mathrm{i}}+\mathrm{N}_{\mathrm{w}, \mathrm{i}}^{\theta_{\mathrm{y}}} \theta_{\mathrm{y}, \mathrm{i}}\right]+\sum_{\mathrm{i}=1}^{\mathrm{n}} \mathrm{N}_{\mathrm{w}, \mathrm{i}}^{\gamma} \widehat{\gamma}_{\mathrm{x}, \mathrm{i}} \\
\theta_{\mathrm{x}}(\xi, \eta)=\sum_{\mathrm{i}=1}^{\mathrm{n}}\left[\mathrm{N}_{\theta_{\mathrm{x}}, \mathrm{i}}^{\mathrm{w}} \mathrm{w}_{\mathrm{i}}+\mathrm{N}_{\theta_{\mathrm{x}}, \mathrm{i}}^{\theta_{\mathrm{x}}} \theta_{\mathrm{x}, \mathrm{i}}+\mathrm{N}_{\theta_{\mathrm{x}}, \mathrm{i}}^{\theta_{\mathrm{y}}} \theta_{\mathrm{y}, \mathrm{i}}-\mathrm{N}_{\mathrm{i}}^{\mathrm{Q}}\left(\mathrm{s}_{\mathrm{i}} \widehat{\theta}_{\mathrm{y}, \mathrm{i}}\right)\right]+\sum_{\mathrm{i}=1}^{\mathrm{n}} \mathrm{N}_{\theta_{\mathrm{x}}, \mathrm{i}}^{\gamma} \widehat{\gamma}_{\mathrm{x}, \mathrm{i}} \\
\theta_{\mathrm{y}}(\xi, \eta)=\sum_{\mathrm{i}=1}^{\mathrm{n}}\left[\mathrm{N}_{\theta_{\mathrm{y}}, \mathrm{i}}^{\mathrm{w}} \mathrm{w}_{\mathrm{i}}+\mathrm{N}_{\theta_{\mathrm{y}}, \mathrm{i}}^{\theta_{\mathrm{x}}} \theta_{\mathrm{x}, \mathrm{i}}+\mathrm{N}_{\theta_{\mathrm{y}}, \mathrm{i}}^{\theta_{\mathrm{y}}} \theta_{\mathrm{y}, \mathrm{i}}+\mathrm{N}_{\mathrm{i}}^{\mathrm{Q}}\left(\mathrm{s}_{\mathrm{i}} \widehat{\theta}_{\mathrm{y}, \mathrm{i}}\right)\right]+\sum_{\mathrm{i}=1}^{\mathrm{n}} \mathrm{N}_{\theta_{\mathrm{y}}, \mathrm{i}}^{\gamma} \widehat{\gamma}_{\mathrm{x}, \mathrm{i}}
\end{gathered}
$$

where the individual shape functions are provided in Appendix A.

Clearly, linked interpolation is achieved for both $\left(\theta_{\mathrm{x}}, \theta_{\mathrm{y}}\right)$ and $\mathrm{w}$ fields. Similar linked interpolation was considered for only the w field by Zienkiewicz et al. [22] for elements utilising linear/quadratic shape functions, while richer element formulations with quadratic/cubic shape functions are considered in the present work without a significant increase in element degrees of freedom (i.e. only $\widehat{\theta}_{\mathrm{y}, \mathrm{i}}$ and $\widehat{\gamma}_{\mathrm{x}, \mathrm{i}}$ are required per edge). It is also observed that shape functions associated with the shear parameter $\widehat{\gamma}_{\mathrm{x}, \mathrm{i}}$ are zero for a simply supported edge $i-i_{+}$, indicating that this parameter should not be considered for such a case.

In the context of large displacement analysis for small strain problems, the local straindisplacement relationship can be taken linear:

$$
\left\{\begin{array}{c}
\varepsilon_{\mathrm{x}} \\
\varepsilon_{\mathrm{y}} \\
\gamma_{\mathrm{xy}}
\end{array}\right\}=\left\{\begin{array}{c}
\frac{\partial \mathrm{u}}{\partial \mathrm{x}} \\
\frac{\partial \mathrm{v}}{\partial \mathrm{y}} \\
\frac{\partial \mathrm{u}}{\partial \mathrm{y}}+\frac{\partial \mathrm{v}}{\partial \mathrm{x}}
\end{array}\right\},\left\{\begin{array}{c}
\kappa_{\mathrm{x}} \\
\kappa_{\mathrm{y}} \\
\kappa_{\mathrm{xy}}
\end{array}\right\}=\left\{\begin{array}{c}
\frac{\partial \theta_{\mathrm{x}}}{\partial \mathrm{x}} \\
\frac{\partial \theta_{\mathrm{y}}}{\partial \mathrm{y}} \\
\frac{\partial \theta_{\mathrm{x}}}{\partial \mathrm{y}}+\frac{\partial \theta_{\mathrm{y}}}{\partial \mathrm{x}}
\end{array}\right\},\left\{\begin{array}{l}
\gamma_{\mathrm{xz}} \\
\gamma_{\mathrm{yz}}
\end{array}\right\}=\left\{\begin{array}{c}
-\theta_{\mathrm{x}}+\frac{\partial \mathrm{w}}{\partial \mathrm{x}} \\
-\theta_{\mathrm{y}}+\frac{\partial \mathrm{w}}{\partial \mathrm{y}}
\end{array}\right\}
$$

with geometric nonlinearity considered at the level of the discrete nodal transformations between the local and global systems, as presented in Section 6.

The shape functions for $\left(\mathrm{w}, \theta_{\mathrm{x}}, \theta_{\mathrm{y}}\right)$ of the triangular element can represent the zero $\gamma /$ constant $\kappa$ only along the element edges. Within the element domain, cubic terms in $\mathrm{w}$ appear, which lead to non-zero $\gamma$, and hence the constant $\kappa$ patch tests cannot be passed. Although the quadrilateral element with a parallelogram geometry can satisfy the zero $\gamma /$ constant $\boldsymbol{\kappa}$ patch test requirement, for a general quadrilateral geometry the shape functions 
for $\left(\mathrm{w}, \theta_{\mathrm{x}}, \theta_{\mathrm{y}}\right)$ are not sufficient for passing the constant $\mathrm{\kappa}$ patch tests. Accordingly, a nonconforming formulation is employed in which a substitute shear strain field is prescribed in terms of the tangential shear strains $\hat{\gamma}_{\mathrm{x}, \mathrm{i}}$. The substitute tangential shear strain field is obtained by firstly transforming $\widehat{\gamma}_{\mathrm{x}, \mathrm{i}}$ for all edges into nodal $\mathrm{x}$ and $\mathrm{y}$ components and then interpolating the nodal values over the element domain. Accordingly, the nodal shear strains $\gamma_{c}$ are related to the element parameters $\mathbf{u}^{\mathrm{e}}$ by:

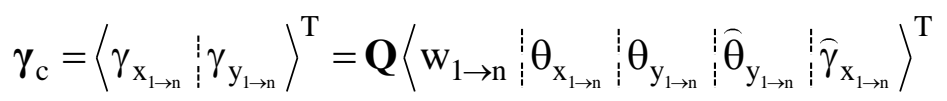

where the terms of $\mathbf{Q}$, taking into account the effect of straight edge constraints, are provided in Appendix B.

The transverse shear strain fields $\gamma$ are then interpolated linearly in terms of $\gamma_{c}$, thus maintaining a compatible distribution of tangential shear strain along the edges even for simply supported edges. The corresponding relationship between $\gamma$ and $\mathbf{u}^{\mathrm{e}}$ is given by:

$$
\boldsymbol{\gamma}=\mathbf{S} \mathbf{u}^{\mathrm{e}}=\left(\left[\begin{array}{ccc:ccc}
\mathrm{L}_{1} & \cdots & \mathrm{L}_{\mathrm{n}} & 0 & \cdots & 0 \\
\hdashline 0 & \cdots & 0 & \mathrm{~L}_{1} & \cdots & \mathrm{L}_{\mathrm{n}}
\end{array}\right] \mathbf{Q}\right) \mathbf{u}^{\mathrm{e}}
$$

which is employed for the evaluation of shear strains within the element domain.

By assuming a non-conforming formulation in which the transverse shear strains $\left(\gamma_{\mathrm{xz}}, \gamma_{\mathrm{yz}}\right)$ over the element domain are obtained using a substitute shear field in terms of $\hat{\gamma}_{\mathrm{x}, \mathrm{i}}$ only [23], a mixed Reissner-Mindlin formulation arises. Importantly, setting the $\widehat{\gamma}_{x, i}$ parameters to zero derives a discrete Kirchhoff formulation [24] that would be applicable to thin plate analysis.

\subsection{Curved shell elements}

The proposed co-rotational systems are also applicable to curved higher-order triangular and quadrilateral shell elements by defining the local system based on corner nodes only. Herein, the local kinematics of curved Reissner-Mindlin shell elements based on the MITC formulations for 9-noded quadrilateral and 6-noded triangular elements, MITC9 [25] and MITC6 [26], are briefly introduced.

The MITC plate and shell elements have been widely employed in addressing locking phenomena. The general MITC formulation procedure can be summarised as follows: 
(i) Evaluate Green strains directly from displacement fields at a set of prescribed tying points.

(ii) Transform the extracted Green strains into corresponding covariant strain components using (33):

$$
\boldsymbol{\varepsilon}_{2}=\mathbf{J}^{\mathrm{T}} \mathbf{E}_{2} \mathbf{J}
$$

where $\mathbf{E}_{2}$ is the Green strain tensor in terms of Cartesian coordinates; $\boldsymbol{\varepsilon}_{2}$ is the covariant strain tensor; and $\mathbf{J}$ is the Jacobian matrix, which is expressed as:

$$
\mathbf{J}=\left[\begin{array}{lll}
\frac{\partial \mathrm{x}}{\partial \xi} & \frac{\partial \mathrm{x}}{\partial \eta} & \frac{\partial \mathrm{x}}{\partial \zeta} \\
\frac{\partial \mathrm{y}}{\partial \xi} & \frac{\partial \mathrm{y}}{\partial \eta} & \frac{\partial \mathrm{y}}{\partial \zeta} \\
\frac{\partial \mathrm{z}}{\partial \xi} & \frac{\partial \mathrm{z}}{\partial \eta} & \frac{\partial \mathrm{z}}{\partial \zeta}
\end{array}\right]
$$

(iii) Interpolate covariant strain fields with the use of the extracted covariant strains:

$$
\varepsilon_{\mathrm{rs}}^{\mathrm{AS}}(\xi, \eta, \zeta)=\mathbf{H}_{\mathrm{rs}} \boldsymbol{\varepsilon}_{\mathrm{rs}, \mathrm{T}}^{\mathrm{DI}}
$$

where: $\mathrm{r}, \mathrm{s}=(\xi, \eta, \zeta)$; the superscript 'AS' and 'DI' refers respectively to the assumed strain distribution and the distribution obtained directly from displacement fields; $\mathbf{H}_{\mathrm{rs}}$ is a row vector of interpolation functions associated with the tying points; $\boldsymbol{\varepsilon}_{\mathrm{rs}, \mathrm{T}}^{\mathrm{DI}}$ consists of the covariant strain values extracted at the tying points.

(iv) Transform the assumed covariant strain fields to the corresponding Green strain fields in terms of real coordinates, obtained from:

$$
\mathbf{E}_{2}^{\mathrm{AS}}=\mathbf{J}^{-\mathrm{T}} \boldsymbol{\varepsilon}_{2}^{\mathrm{AS}} \mathbf{J}^{-1}
$$

(v) Replace displacement-based strains with the assumed strain distributions obtained from (iv) in the element formulation.

The MITC9 [25] quadrilateral shell element (Figure 9) performs well, but it does not pass the patch test for irregular element shapes due to the varying basis used for sampling and mapping covariant strains, which can be resolved by using a constant Jacobian matrix evaluated at the element centre [27]. This is equivalent to replacing (33) and (36) with: 


$$
\begin{gathered}
\boldsymbol{\varepsilon}_{2}=\mathbf{J}_{\mathrm{C}}^{\mathrm{T}} \mathbf{E}_{2} \mathbf{J}_{\mathrm{C}} \\
\mathbf{E}_{2}^{\mathrm{AS}}=\mathbf{J}_{\mathrm{C}}^{-\mathrm{T}} \boldsymbol{\varepsilon}_{2}^{\mathrm{AS}} \mathbf{J}_{\mathrm{C}}^{-1}
\end{gathered}
$$

where $\mathbf{J}_{C}$ is the Jacobian matrix evaluated at the element centre $(\xi=0, \eta=0)$. Similarly, the MITC6 [26] triangular shell element (Figure 10) is enhanced with the same method [28], where $\mathbf{J}_{\mathrm{C}}$ is evaluated at $(\xi=1 / 3, \eta=1 / 3)$.

In this work, Reissner-Mindlin shell elements are developed based on MITC9 and MITC6 local formulations, where five basic nodal parameters (three translations and two rotations) are utilised, as denoted previously by $\mathbf{u}_{\mathrm{b}}$ in (20). The shape functions for isoparametric mapping of the element geometry and local displacement fields are as follows.

For the 9-noded quadrilateral element, the shape functions are expressed in terms of natural coordinates $(\xi, \eta)$ :

$$
\mathrm{N}_{\mathrm{i}}=\frac{\left(\xi-\xi_{\mathrm{i}}^{\prime}\right)\left(\xi-\xi_{\mathrm{i}}^{\prime \prime}\right)}{\left(\xi_{\mathrm{i}}-\xi_{\mathrm{i}}^{\prime}\right)\left(\xi_{\mathrm{i}}-\xi_{\mathrm{i}}^{\prime \prime}\right)} \cdot \frac{\left(\eta-\eta_{\mathrm{i}}^{\prime}\right)\left(\eta-\eta_{\mathrm{i}}^{\prime \prime}\right)}{\left(\eta_{\mathrm{i}}-\eta_{\mathrm{i}}^{\prime}\right)\left(\eta_{\mathrm{i}}-\eta_{\mathrm{i}}^{\prime \prime}\right)} \quad(\mathrm{i}=1 \rightarrow 9)
$$

where $\left(\xi_{\mathrm{i}}, \eta_{\mathrm{i}}\right)$ represents the natural coordinates of node $i$; $\left(\xi_{\mathrm{i}} \neq \xi_{\mathrm{i}}{ }^{\prime} \neq \xi_{\mathrm{i}}{ }^{\prime \prime}\right)=-1,0,1$; and $\left(\eta_{\mathrm{i}} \neq \eta_{\mathrm{i}}^{\prime} \neq \eta_{\mathrm{i}}^{\prime \prime}\right)=-1,0,1$.

For the 6-noded triangular element, the shape functions are first expressed in terms of area coordinates $\left(\mathrm{L}_{1}, \mathrm{~L}_{2}, \mathrm{~L}_{3}\right)$ :

$$
\mathrm{N}_{\mathrm{i}}=\mathrm{L}_{\mathrm{i}}\left(2 \mathrm{~L}_{\mathrm{i}}-1\right), \quad \mathrm{N}_{\mathrm{i}+3}=4 \mathrm{~L}_{\mathrm{i}} \mathrm{L}_{\mathrm{i}+} \quad(\mathrm{i}=1 \rightarrow 3)
$$

and are then expressed in terms of natural coordinates $(\xi, \eta)$ by setting $L_{1}=1-\xi-\eta, L_{2}=\xi$, and $\mathrm{L}_{3}=\eta$ (Figure 10).

The element strain state is fully determined by membrane strains $\varepsilon^{\mathrm{m}}$, bending strains $\boldsymbol{\varepsilon}^{\mathrm{b}}$, and transverse shear strains $\boldsymbol{\varepsilon}^{\mathrm{s}}$. Although a geometric linear local element formulation may be used within the co-rotational framework for geometric nonlinear analysis, a quadratic strain-displacement relationship based on von Karman's theory is adopted to achieve improved accuracy with coarser meshes, where the various generalised strains are as follows: 


$$
\begin{aligned}
& \boldsymbol{\varepsilon}^{\mathrm{m}}=\left\{\begin{array}{c}
\varepsilon_{\mathrm{x}} \\
\varepsilon_{\mathrm{y}} \\
\gamma_{\mathrm{xy}}
\end{array}\right\}=\left\{\begin{array}{c}
\frac{\partial \mathrm{u}}{\partial \mathrm{x}} \\
\frac{\partial \mathrm{v}}{\partial \mathrm{y}} \\
\frac{\partial \mathrm{u}}{\partial \mathrm{y}}+\frac{\partial \mathrm{v}}{\partial \mathrm{x}}
\end{array}\right\}+\left\{\begin{array}{c}
\frac{1}{2}\left(\frac{\partial \mathrm{z}}{\partial \mathrm{x}}+\frac{\partial \mathrm{w}}{\partial \mathrm{x}}\right)^{2}-\frac{1}{2}\left(\frac{\partial \mathrm{z}}{\partial \mathrm{x}}\right)^{2} \\
\frac{1}{2}\left(\frac{\partial \mathrm{z}}{\partial \mathrm{y}}+\frac{\partial \mathrm{w}}{\partial \mathrm{y}}\right)^{2}-\frac{1}{2}\left(\frac{\partial \mathrm{z}}{\partial \mathrm{y}}\right)^{2} \\
\left(\frac{\partial \mathrm{z}}{\partial \mathrm{x}}+\frac{\partial \mathrm{w}}{\partial \mathrm{x}}\right)\left(\frac{\partial \mathrm{z}}{\partial \mathrm{y}}+\frac{\partial \mathrm{w}}{\partial \mathrm{y}}\right)-\left(\frac{\partial \mathrm{z}}{\partial \mathrm{x}}\right)\left(\frac{\partial \mathrm{z}}{\partial \mathrm{y}}\right)
\end{array}\right\} \\
& \boldsymbol{\varepsilon}^{b}=\left\{\begin{array}{c}
\kappa_{\mathrm{x}} \\
\kappa_{\mathrm{y}} \\
\kappa_{\mathrm{xy}}
\end{array}\right\}=\left\{\begin{array}{c}
\frac{\partial \theta_{\mathrm{x}}}{\partial \mathrm{x}} \\
\frac{\partial \theta_{\mathrm{y}}}{\partial \mathrm{y}} \\
\frac{\partial \theta_{\mathrm{x}}}{\partial y}+\frac{\partial \theta_{\mathrm{y}}}{\partial \mathrm{x}}
\end{array}\right\} \\
& \boldsymbol{\varepsilon}^{\mathrm{s}}=\left\{\begin{array}{l}
\gamma_{\mathrm{xz}} \\
\gamma_{\mathrm{yz}}
\end{array}\right\}=\left\{\begin{array}{l}
-\theta_{\mathrm{x}}+\frac{\partial \mathrm{w}}{\partial \mathrm{x}} \\
-\theta_{\mathrm{y}}+\frac{\partial \mathrm{w}}{\partial \mathrm{y}}
\end{array}\right\}
\end{aligned}
$$

Before employing the tying schemes for the element formulation, further assumptions are made that the element is shallow and thin, so that the natural coordinate axis $\zeta$ is taken to have an identical orientation to the local z-axis, and the transverse normal strain $\varepsilon_{\mathrm{z}}$ is ignored. Accordingly, the Jacobian matrix is simplified to:

$$
\mathbf{J}=\left[\begin{array}{ccc}
\frac{\partial \mathrm{x}}{\partial \xi} & \frac{\partial \mathrm{x}}{\partial \eta} & 0 \\
\frac{\partial \mathrm{y}}{\partial \xi} & \frac{\partial \mathrm{y}}{\partial \eta} & 0 \\
0 & 0 & \frac{\mathrm{t}}{2}
\end{array}\right]
$$

where $t$ is the shell thickness. This simplification enables decoupled relationships between real strains and covariant strains, with each set of the generalised real strains related to their covariant counterparts only, which are expressed thus as:

$$
\left[\begin{array}{cc}
\varepsilon_{\xi \xi} & \varepsilon_{\xi \eta} \\
\varepsilon_{\xi \eta} & \varepsilon_{\eta \eta}
\end{array}\right]=\mathbf{J}_{\mathrm{p}}^{\mathrm{T}}\left[\begin{array}{cc}
\varepsilon_{\mathrm{x}} & \frac{1}{2} \gamma_{\mathrm{xy}} \\
\frac{1}{2} \gamma_{\mathrm{xy}} & \varepsilon_{\mathrm{y}}
\end{array}\right] \mathbf{J}_{\mathrm{p}}
$$




$$
\begin{gathered}
{\left[\begin{array}{cc}
\kappa_{\xi \xi} & \kappa_{\xi \eta} \\
\kappa_{\xi \eta} & \kappa_{\eta \eta}
\end{array}\right]=\mathbf{J}_{\mathrm{p}}^{\mathrm{T}}\left[\begin{array}{cc}
\kappa_{\mathrm{x}} & \frac{1}{2} \kappa_{\mathrm{xy}} \\
\frac{1}{2} \kappa_{\mathrm{xy}} & \kappa_{\mathrm{y}}
\end{array}\right] \mathbf{J}_{\mathrm{p}}} \\
\left\{\begin{array}{c}
\varepsilon_{\xi \zeta} \\
\varepsilon_{\eta \zeta}
\end{array}\right\}=\frac{\mathrm{t}}{2} \mathbf{J}_{\mathrm{p}}^{\mathrm{T}}\left\{\begin{array}{l}
\frac{1}{2} \gamma_{\mathrm{xz}} \\
\frac{1}{2} \gamma_{\mathrm{yz}}
\end{array}\right\}
\end{gathered}
$$

where $\mathbf{J}_{\mathrm{p}}$ is a sub-matrix of $\mathbf{J}$ :

$$
\mathbf{J}_{\mathrm{p}}=\left[\begin{array}{ll}
\frac{\partial \mathrm{x}}{\partial \xi} & \frac{\partial \mathrm{x}}{\partial \eta} \\
\frac{\partial \mathrm{y}}{\partial \xi} & \frac{\partial \mathrm{y}}{\partial \eta}
\end{array}\right]
$$

By using (43a-c) and evaluating $\mathbf{J}_{\mathrm{p}}$ at the element centroid, the strains at each tying point are readily transformed to the covariant strains, which are then used in mapping the assumed covariant strain fields. The positions of the tying points for the MITC9 and MITC6 elements are shown in respectively Figure 9 and Figure 10, where the associated interpolation functions for the covariant strains can be found elsewhere $[25,26]$. Once the distribution of the covariant strain fields is obtained, these are transformed back to real assumed strains in the local co-rotational system via the use of (43a-c), and these are then used in the formulation of the local response of the 6-noded and 9-noded curved shell elements.

\section{LARGE DISPLACEMENT TRANSFORMATIONS}

The proposed bisector and zero-macrospin definitions of the local co-rotational system benefit from nodal invariance, and importantly they are completely independent of the local element formulation including it order. This is illustrated here in the derivation of the large displacement transformations relating local and global element entities, which are generically applicable to the different local formulations of flat and curved shell elements of a specific triangular or quadrilateral shape, as presented in the previous section. 
Local nodal translations are established from the global translational degrees of freedom by rotating the initial undeformed configuration about the origin of $\left(\mathbf{c}_{\mathrm{x}}^{\mathrm{o}}, \mathbf{c}_{\mathrm{y}}^{\mathrm{o}}, \mathbf{c}_{\mathrm{z}}^{\mathrm{o}}\right)$ to the current local system orientation, as defined by $\left(\mathbf{c}_{\mathrm{x}}, \mathbf{c}_{\mathrm{y}}, \mathbf{c}_{\mathrm{z}}\right)$, and then measuring the translations from the rotated undeformed configuration. On the other hand, the local nodal rotations are determined as the projection of the nodal normals on the rotated local reference system $\left(\mathbf{c}_{\mathrm{x}}, \mathbf{c}_{\mathrm{y}}, \mathbf{c}_{\mathrm{z}}\right)$. For smooth surfaces where normals are uniquely defined over the domain, only two global rotational parameters are required for each node. Therefore, the two smallest components of each nodal normal are directly used as global rotational degrees of freedom when dealing with finite rotations of the normal [18], resulting in five global parameters per node:

$$
\mathbf{U}_{\mathrm{Gi}}=\left\langle\mathbf{d}_{\mathrm{i}}^{\mathrm{T}} \overline{\mathbf{n}}_{\mathrm{i}}^{\mathrm{T}}\right\rangle^{\mathrm{T}}
$$

where $\mathbf{d}_{\mathrm{i}}$ represents the global translational displacements of node $i$, as defined in Section 3.1, $\mathbf{n}_{\mathrm{i}}$ is the normal vector at node $i$, and $\overline{\mathbf{n}}_{\mathrm{i}}=\left\langle\mathbf{n}_{\mathrm{i}, \alpha_{\mathrm{i}}} \mathbf{n}_{\mathrm{i}, \beta_{\mathrm{i}}}\right\rangle^{\mathrm{T}}$ represents the two components of $\mathbf{n}_{\mathrm{i}}$ which are smallest in absolute terms, the third component $\mathbf{n}_{\mathrm{i}, \gamma_{\mathrm{i}}}$ being determined by the constraint of a unit $\mathbf{n}_{\mathrm{i}}$. The indices $\alpha_{\mathrm{i}}, \beta_{\mathrm{i}}$ and $\gamma_{\mathrm{i}}$ are established from the following condition:

$$
\left|\mathbf{n}_{\mathrm{i}, \alpha_{\mathrm{i}}}^{\mathrm{o}}\right| \leq\left|\mathbf{n}_{\mathrm{i}, \beta_{\mathrm{i}}}^{\mathrm{o}}\right| \leq\left|\mathbf{n}_{\mathrm{i}, \gamma_{\mathrm{i}}}^{\mathrm{o}}\right|
$$

where $\mathbf{n}_{\mathrm{i}}^{\mathrm{o}}$ is the normal vector of the last known equilibrium configuration. Therefore, the indices $\alpha_{i}, \beta_{i}$, and $\gamma_{i}$ are revised at the beginning of each step.

Accordingly, the three components of the normal can be obtained from the two parameters $\left(\mathbf{n}_{\mathrm{i}, \alpha_{\mathrm{i}}}, \mathbf{n}_{\mathrm{i}, \beta_{\mathrm{i}}}\right)$ as:

$$
\mathbf{n}_{i, \alpha_{i}}=\overline{\mathbf{n}}_{i, 1}, \quad \mathbf{n}_{i, \beta_{i}}=\overline{\mathbf{n}}_{i, 2}, \quad \mathbf{n}_{i, \gamma_{i}}=\operatorname{sign}\left(\mathbf{n}_{i, \gamma_{i}}^{o}\right) \sqrt{1-\overline{\mathbf{n}}_{\mathrm{i}}^{\mathrm{T}} \overline{\mathbf{n}}_{\mathrm{i}}}
$$

It is noted here that the case of shell elements meeting at an angle can be dealt with using 3 global nodal rotational parameters at the intersections, which define the normal vectors $\mathbf{n}_{\mathrm{i}}$ using a suitable rotation matrix, such as the quadratic incremental rotation matrix previously proposed by the first author [29].

In the co-rotational system, there are five local basic parameters per node: 


$$
\mathbf{U}_{\mathrm{Ci}}=\left\langle\mathbf{t}_{\mathrm{i}}^{\mathrm{T}} \overline{\mathbf{r}}_{\mathrm{i}}^{\mathrm{T}}\right\rangle^{\mathrm{T}}
$$

where: $\mathbf{t}_{\mathrm{i}}=\left\langle\mathrm{u}_{\mathrm{i}}, \mathrm{v}_{\mathrm{i}}, \mathrm{w}_{\mathrm{i}}\right\rangle^{\mathrm{T}}$ represents the local translational displacements of node $i$; $\overline{\mathbf{r}}_{\mathrm{i}}=\left\langle\theta_{\mathrm{x}, \mathrm{i}}, \theta_{\mathrm{y}, \mathrm{i}}\right\rangle^{\mathrm{T}}$ represents the two rotations at node $i$ in the local $\mathrm{x}-\mathrm{z}$ and $\mathrm{y}-\mathrm{z}$ planes, respectively.

Note that $\mathbf{U}_{\mathrm{Ci}}$ represents the basic local nodal parameters, defined as $\mathbf{u}_{\mathrm{b}}$ in (26), associated with node $i$, which are obtained from the corresponding global parameters $\mathbf{U}_{\mathrm{Gi}}$ using co-rotational transformations. For the flat shell elements of Section 5.1, which use additional hierarchic degrees of freedom $\mathbf{u}_{\mathrm{a}}$ at edges, the four hierarchic parameters are directly shared between adjacent elements, and as such they are excluded from the corotational transformations.

For both flat and curved shell elements, the transformation between global and local translational displacements can be expressed as [18]:

$$
\mathbf{t}_{\mathrm{i}}=\mathbf{R} \mathbf{d}_{\mathrm{i}}+\left(\mathbf{R}-\mathbf{R}^{\mathrm{o}}\right) \mathbf{v}_{\mathrm{io}}^{\mathrm{o}} \quad(\mathrm{i}=1 \rightarrow \mathrm{n})
$$

where $\mathbf{R}^{\mathrm{o}}$ and $\mathbf{R}$ are the orientation matrices of the local co-rotational system in the initial and current configurations, respectively, defined as:

$$
\mathbf{R}^{\mathrm{o}}=\left[\begin{array}{lll}
\mathbf{c}_{\mathrm{x}}^{\mathrm{o}} & \mathbf{c}_{\mathrm{y}}^{\mathrm{o}} & \mathbf{c}_{\mathrm{z}}^{\mathrm{o}}
\end{array}\right]^{\mathrm{T}}, \quad \mathbf{R}=\left[\begin{array}{lll}
\mathbf{c}_{\mathrm{x}} & \mathbf{c}_{\mathrm{y}} & \mathbf{c}_{\mathrm{z}}
\end{array}\right]^{\mathrm{T}}
$$

and $\mathbf{v}_{\mathrm{io}}^{\mathrm{o}}$ is a vector describing the initial position of node $i$ relative to a reference point $o$, which in turn represents the centre of rotation of the initial configuration to align with the current configuration. For triangular elements, the reference point o is selected as node 1, while for quadrilateral elements the intersection of the diagonals and the internal node are selected for the 4-noded and 9-noded shell elements, respectively. Nevertheless, it should be noted that choice of the reference point does not impact on accuracy, since a change in location of $o$ merely results in the addition of an increment of rigid body translations to which the local element formulation should be insensitive.

The transformation between global and local rotations for both triangular and quadrilateral elements is [18]:

$$
\overline{\mathbf{r}}_{\mathrm{i}}=-\overline{\mathbf{R}} \mathbf{n}_{\mathrm{i}}, \quad \overline{\mathbf{R}}=\left[\begin{array}{ll}
\mathbf{c}_{\mathrm{x}} & \mathbf{c}_{\mathrm{y}}
\end{array}\right]^{\mathrm{T}} \quad(\mathrm{i}=1 \rightarrow \mathrm{n})
$$


Note that the current orientation of the local reference system $\left(\mathbf{c}_{\mathrm{x}}, \mathbf{c}_{\mathrm{y}}, \mathbf{c}_{\mathrm{z}}\right)$ depends on the adopted definition, whether bisector or zero-macrospin, as discussed in Sections 3 and 4.

The remaining transformations between the local co-rotational and global reference systems relate to the determination of the global nodal forces and tangent stiffness matrix from the corresponding local entities, requiring the first and second partial derivatives of local parameters with respect to global parameters. These derivatives depend in turn on the first and second partial derivatives of $\left(\mathbf{c}_{\mathrm{x}}, \mathbf{c}_{\mathrm{y}}, \mathbf{c}_{\mathrm{z}}\right)$ with respect to global translational degrees of freedom, where the first derivatives are given in Appendix $\mathrm{C}$ and the second derivatives can be similarly derived. It is emphasised that the resulting tangent stiffness matrix is always symmetric for all proposed definitions of the local co-rotational system, since in all cases the triad $\left(\mathbf{c}_{\mathrm{x}}, \mathbf{c}_{\mathrm{y}}, \mathbf{c}_{\mathrm{z}}\right)$ is explicitly, thus uniquely, related to the global translational parameters, leading to explicit relationships between the local and global parameters.

\section{NUMERICAL EXAMPLES}

The large displacement co-rotational approach based on the local system definitions proposed in Sections 3 and 4 has been implemented in ADAPTIC [30] v2.12.9 for the flat and curved shell elements introduced in Section 5. Several numerical examples are considered hereafter employing ADAPTIC to assess the relative accuracy of the various definitions, using in all cases Reissner-Mindlin elements. The simplest local system for both the triangular and quadrilateral elements, namely that following the first element edge and violating nodal invariance as described in Section 2, is also considered here for comparison. In presenting the results, the various local system definitions and element types are referred to by element codes, as listed in Table 1 .

\subsection{Clamped strip under torsion}

A flat strip, fully clamped at one end, is subjected to a force couple at the free end, as depicted in Figure 11. The geometric and material parameters are given as $\mathrm{L}=12, \mathrm{w}=1$, $\mathrm{h}=0.1, \mathrm{E}=1.2 \times 10^{6}$, and $v=0.0$.

The strip is first modelled with quadrilateral elements using $12 \times 1$ and $24 \times 2$ meshes. Figure 12 shows the deflected shape of the strip for a $24 \times 2$ mesh. The equilibrium paths of 
the z-displacement at Point A with different co-rotational systems are plotted in Figures 13 and 14 for two respective meshes. Four alternative co-rotational systems are employed for the first-edge definition, in which the x-axes of FEQ-1 FEQ-4 are aligned respectively with edge 1-2, 2-3, 3-4, and 4-1 as denoted in Figure 11. It is emphasized that the results with the BSQ and the ZMQ definitions are completely insensitive to nodal ordering, in the sense that identical predictions are obtained regardless of the order in which the element nodes are specified. It is observed from Figure 13 that the BSQ and ZMQ definitions yield the same results, which are consistent with the results from a finer mesh $(24 \times 2)$ with the ZMQ definition. However, the mesh with four FEQ co-rotational systems leads to different equilibrium paths, owing to the significant twisting involved in this large displacement problem, as explained in Section 2. Due to the element twisting, the four nodes in the deflected element configuration are not in the same plane, thus leading to results with the FEQ definition, which incorporates only three nodes in determining the element normal, highly dependent on nodal ordering. By contrast, the two proposed co-rotational systems, BSQ and ZMQ, use all four nodes in the determination of the element plane, thus achieving nodal invariance and leading to more accurate predictions. Figure 14 shows the results for the finer mesh $(24 \times 2)$, in which the element twisting is reduced with mesh refinement for all corotational system definitions. It is observed that the deviation of the FEQ results from the $\mathrm{BSQ} / \mathrm{ZMQ}$ results is reduced, though there are still significant discrepancies for a relatively fine mesh, particularly at large displacements. This confirms the importance of nodal invariance for small strain plate/shell problems in the presence of twisting, as noted in Section 2.

The strip is next modelled with triangular elements using a $12 \times 1$ mesh (see Figure 11) and considering the various co-rotational systems. Also provided in the figure is the result from a $12 \times 1$ mesh of quadrilateral elements with the zero-macrospin co-rotational approach. In this example, changing the order of element nodes has little influence on the results with the first edge co-rotational approach, so only one FET curve is plotted against the ZMT and the BST curves. Figure 15 indicates that the three definitions of the co-rotational approach for the triangular element lead to almost identical results throughout the large-displacement small- 
strain range, owing to the fact that in all of the three definitions the element normal is correctly defined. In addition, the mesh of triangular elements with various local co-rotational approaches yields results very close to that of the mesh with quadrilateral elements.

\subsection{Annular plate under transverse end shear}

An isotropic annular plate, which is fully clamped at one end, is subjected to a uniformly distributed transverse load at the other end, as is shown in Figure 16. The geometric and material properties are specified as: $\mathrm{R}_{1}=6, \mathrm{R}_{2}=10, \mathrm{~h}=0.03, \mathrm{E}=21.0 \times 10^{6}$, and $v=0.0$.

The whole plate is first modelled with $24 \times 4$ and $48 \times 8$ meshes of the quadrilateral element with various co-rotational systems, and the displacement in the $\mathrm{z}$ direction at point $\mathrm{A}$ is investigated. The deformed configuration with the $24 \times 4$ mesh is shown in Figure 17 .

This is again a characteristic shell problem involving significant twisting. Two alternatives of the first-edge co-rotational system definition are considered, in which the local x-axes of FEQ-1 and FEQ-2 orient respectively to the circumferential and the radial directions. Figure 18 depicts the load-displacement curves for the coarser $24 \times 4$ mesh, and the ZMQ solution with a $96 \times 16$ mesh is used as a reference solution. Similar to the previous example, the BSQ and the ZMQ curves coincide and are very close to the reference solution, while the FEQ results show significant errors at large displacements. Also shown in the figure are the results of models by Arciniega and Reddy [31] and Sze et al. [32], both of which utilise a comparable number of degrees of freedom (see Table 2). The ZMQ and BSQ solutions compare well against the results by other researchers [31,32], indicating the reliability of both proposed co-rotational approaches. The discrepancy of the FEQ system is reduced with the finer $48 \times 8$ mesh though it is still tangible, as illustrated in Figure 19 .

The annular plate is next modelled with triangular elements using a $24 \times 4$ mesh, as shown in Figure 16. Also depicted in the figure is the solution from a $24 \times 4$ mesh of quadrilateral elements with the zero-macrospin approach employed. The load-displacement curves corresponding to the three definitions of the co-rotational systems for the triangular element again coincide throughout the large-displacement small-strain range, and are almost identical to the solution of a quadrilateral element mesh, as shown in Figure 20. 
Together with the previous example, the obtained results demonstrate the effectiveness of the proposed bisector and the zero-macrospin co-rotational systems in plate/shell problems involving significant twisting. The importance of the invariance characteristic is particularly manifested for quadrilateral elements. With the participation of the four corner nodes in determining the orientation of the co-rotational system, including the effective normal, nodal invariance is obtained, and accurate results are achieved for relatively coarse meshes. Since the effective normal is uniquely defined for the triangular element, regardless of the corotational system definition, the discrepancy between the various definitions is significantly reduced, as anticipated in Section 2 for small strain problems.

\subsection{Skew plate under point load}

Consideration is given here to a thin skew plate, clamped along two opposite edges and free along the remaining two edges, which is subjected to a point load $\mathrm{P}$ applied at the plate centre, as depicted in Figure 21, with the deflected configuration under loading shown in Figure 22. The geometry and material properties for the plate are: $\mathrm{L}=2, \alpha=60^{\circ}, \mathrm{h}=0.1$, $\mathrm{E}=1.2 \times 10^{6}$, and $v=0.25$.

Figure 23 shows the load-centre deflection curves for $16 \times 8$ and $32 \times 16$ meshes of the quadrilateral elements using different co-rotational systems. Two cases of the first edge corotational system definition are chosen such that the x-axes of FEQ-1 and FEQ-2 are parallel to the free edge and the clamped edge, respectively. It is observed that the BSQ and ZMQ results for each mesh are identical for the considered meshes where the element aspect ratio is 1.0, and convergence is exhibited with relatively small errors for the coarser mesh. By contrast, the FEQ solution is highly dependent on nodal ordering in the sense that the two FEQ curves are quite different, with the FEQ-1 results terminating at a displacement of around 1.0 due to excessive local twisting and the FEQ-2 results deviating significantly from the converged BSQ and ZMQ results.

To investigate the effect of element aspect ratio on the relative accuracy of the ZMQ and the BSQ approaches, a $16 \times 16$ mesh of quadrilateral elements, corresponding to an element aspect ratio $(\mathrm{r}=2)$, is employed for the two approaches, where the predicted response in the 
range of large displacements (1.0 to 1.8) is compared in Figure 24. Also incorporated are the solutions using $16 \times 8$ and $32 \times 16$ meshes with a unit element aspect ratio $(r=1)$, which bound the solution with the $16 \times 16$ ZMQ element mesh. Similarly, Figure 25 presents the solutions using a $16 \times 32$ mesh, corresponding to a more elongated element aspect ratio $(r=4)$, with the results of $16 \times 8$ and $64 \times 32$ meshes again bounding the $16 \times 32$ ZMQ solution. From both sets of results, it is notable that a discrepancy arises between the bisector and zero-macrospin definitions for elongated elements at large displacements, with the discrepancy increasing with element elongation. Although it is arguable whether a linear local element formulation is realistic for this problem in the range of very large displacements, given that significant planar strains are induced, it is evident that the zero-macrospin definition offers a more consistent approach than the bisector definition, in relation to element aspect ratio, that is applicable for a wider range of strains. This is attributed to the ability of the zero-macrospin approach to minimise material spin over the local element domain regardless of the element aspect ratio.

Finally, the skew plate is modelled with triangular elements using $16 \times 16$ and $32 \times 32$ meshes, with a mesh pattern similar to that shown in Figure 21 . Two first-edge corotational system definitions are considered, in which the x-axes of FET-1 and FET-2 are parallel to the free and clamped edges, respectively. It is observed from Figure 26 that the BST and ZMT solutions for each of the meshes are virtually identical, suggesting that the difference between the two proposed definitions is not significant for triangular elements. In contrast, the FET solutions deviate from each other and from the BST/ZMT solutions, indicating dependence of the FET results on nodal ordering and their inaccuracy in the range of moderate displacements. This inaccuracy arises from the fact that material spin cannot be generally reduced with a first-edge definition for the co-rotational system and strongly depends on nodal ordering, as previously discussed in Section 2.

\subsection{Hinged cylindrical shell under a point load}

A cylindrical shell, hinged along its two straight edges, is subjected to a central point load $\mathrm{F}$, as depicted in Figure 27. The shell has a radius $\mathrm{R}=2540$, half-length $\mathrm{L}=254$, and half- 
substending angle $\beta=0.1 \mathrm{rad}$. In this problem, two alternative shell thicknesses, $t=12.7$ and 6.35, are considered. The isotropic material properties are $E=3.105$ and $v=0.3$. In this problem, the curved shell elements BSQ2 and ZMT2 are employed in separate models to demonstrate the effectiveness of the proposed co-rotational systems. Due to symmetry, a quarter of the shell is modelled using the BSQ2 and the ZMT2 elements with different mesh sizes. Figure 27 shows a $3 \times 3$ mesh of ZMT2 elements.

The central load-deflection curves for the two different shell thicknesses are plotted in Figures 28 and 29. Also presented are the convergent results by Li et al. [3] who used a $3 \times 3$ mesh of 6-noded elements, and Kim et al. [8] who used a $4 \times 4 \times 2$ mesh of 8 -noded degenerated solid-shell elements. Clearly, the $2 \times 2$ mesh of BSQ2 gives a convergent solution for both thicknesses considered, while the ZMT2 results is convergent with respectively a $2 \times 2$ and a $3 \times 3$ mesh for the two thicknesses. Note that for both BSQ2 and ZMT2 models, the predicted response remains unchanged with different nodal ordering, and the predictions for the two thicknesses compare well with the results of others $[3,8]$.

\subsection{Pinched hemispherical shell}

A hemispherical shell, with a radius of 10 and thickness of 0.5 , is subjected to an orthogonal set of two inward and two outward forces, as depicted in Figure 30. The material response is assumed to be elasto-plastic with a von Mises yield criterion and isotropic strain hardening, where $\mathrm{E}=1.0 \times 10^{1}, v=0.2, \mathrm{f}_{\mathrm{y}}=0.2$, and $\mathrm{H}=9.0$, in which $\mathrm{H}$ is a constant hardening parameter associated with equivalent plastic strains. Due to symmetry, only a quarter of the hemisphere is modelled, where the curved shell elements BSQ2 and ZMT2 are employed to demonstrate the effectiveness of the proposed co-rotational system definitions. The discretisation patterns for the quadrilateral and the triangular elements are illustrated in Figure 31, while Figure 32 depicts the deformed configuration of the pinched hemisphere.

For both element types, $6 \times 6$ and $12 \times 12$ meshes are employed for the quarter model in each of the three subdomains, and 5 Gauss points are employed over the thickness to capture the spread of material plasticity. The load-deflection curves at the pinching points A and B for BSQ2 and ZMT2 are plotted in Figures 33 and 34, respectively, where it is evident that a $6 \times 6$ 
mesh provides a convergent solution for both the BSQ2 and ZMT2 elements. Also shown in the figures are results from Bestch and Stein [33] using three subdomains of 16×16 4-noded quadrilateral elements, Eberlein and Wriggers [7] using three subdomains of $12 \times 12$ 4-noded quadrilateral elements, and $\mathrm{Li}$ et al. [4] using three subdomains of $6 \times 6$ 6-noded triangular elements. Note that the number of nodes in a $6 \times 6$ mesh of either 9 -noded or 6-noded elements equals that in a $12 \times 12$ mesh of 4 -noded elements. Therefore, all the reference results have comparable number of nodes with the $6 \times 6$ mesh of BSQ2 and ZMT2. Evidently, the BSQ2 and ZMT2 curves match well with the results of Bestch and Stein [33] and Eberlein and Wriggers [7], thus verifying the accuracy of the co-rotational system definitions.

As in previous examples, changing node numbering yields identical results for both the BSQ2 and ZMT2 models. Table 3 lists the predicted deflections at pinched points A and B under $\mathrm{F}=0.03$ with a $6 \times 6$ mesh of various elements, considering the two nodal ordering sequences shown in Figure 35. The MH6T results are given by Li et al. [4], who used the first-edge definition of the co-rotational system for the 6-noded element. As evident from these results, the BSQ2 and ZMT2 results are invariant to element nodal ordering, in contrast with the MH6T results which show a discernible difference for the two nodal ordering sequences.

\section{CONCLUSIONS}

This paper proposes two effective definitions of the local co-rotational approach, both of which are applicable to triangular and quadrilateral elements, and both satisfying the requirements of i) being simple to establish, ii) achieving nodal invariance, iii) reducing the spin of the element, and iv) providing a symmetric element tangent stiffness matrix.

Although the significance of nodal invariance for the co-rotational approach in the context of large strain problems and certain stability problems have been previously recognised, its importance in small strain problems has been largely discounted. This work demonstrates there is a class of plate bending problems with significant twisting that pose additional specific requirements on this invariance characteristic in large displacement analysis even for small strains. Within this context, two efficient definitions of the co-rotational approach, 
namely the bisector definition and the zero-macrospin definition, are proposed for largedisplacement small-strain plate/shell problems.

The bisector definition, previously presented for quadrilateral elements [18], defines the local axes by aligning the bisectors of the two diagonals of the undeformed element with those of the current deformed configuration. This definition is herein elaborated to deal with triangular elements, where the bisectors of the angle that has changed most from the undeformed configuration are aligned. As an alternative to the bisector definition, a zeromacrospin definition is also proposed, which is applicable to both triangular and quadrilateral elements. Based on this definition, the element deformations are obtained from the rotated undeformed configuration using 'stretch' operations only, thus minimising the spin over the element domain.

The two proposed definitions of the co-rotational system enable the local element triad to be obtained directly from global nodal coordinates in the deformed configuration, where the explicit relationship between local and global variables guarantees a symmetric element tangent stiffness matrix. In each of the definitions, all corner nodes are involved in defining the local triad, thus achieving the nodal invariance and independence of the local element formulation and order. In addition, both approaches reduce the spin of the element, where the bisector definition minimises the spin of diagonal angles (for quadrilateral elements) or the internal angle that changes most (for triangular elements), whilst the zero-macrospin definition obtains the element deformation by employing 'stretch' only operations. Therefore, both definitions fulfil all the stated requirements for an effective co-rotational system that is applicable to large-displacement small-strain problems.

Results obtained from several numerical examples demonstrate that both bisector and zero-macrospin definitions of the co-rotational approach address the four requirements well for large-displacement small-strain problems, but that the zero-macrospin definition is more consistent for quadrilateral elements for a wider range of strains. 


\section{REFERENCES}

[1] Rankin CC, Brogan FA. An element independent corotational procedure for the treatment of large rotations. Journal of Pressure Vessel Technology 1986; 108(2):165174.

[2] Crisfield MA, Moita GF. A unified co-rotational framework for solids, shells and beams. International Journal of Solids and Structures 1996; 33(20):2969-2992.

[3] Li ZX, Vu-Quoc L. An efficient co-rotational for curved triangular shell element. International Journal of Numerical Methods in Engineering 2007; 72(9):1029-1062.

[4] Li ZX, Xiang Y, Izzuddin BA, Vu-Quoc L, Zhuo X, Zhang C. A 6-node co-rotational triangular elasto-plastic shell element. Computational Mechanics 2015; DOI: 10.1007/s00466-015-1138-1.

[5] Jiang L, Chernuka MW. A simple four-noded corotational shell element for arbitrarily large rotations. Computers \& Structures 1994; 53(5):1123-1132.

[6] Norachan P, Suthasupradit S, Kim KD. A co-rotational 8-node degenerated thin-walled element with assumed natural strain and enhanced assumed strain. Finite Elements in Analysis and Design 2012; 50:70-85.

[7] Eberlein R, Wriggers P. Finite element concepts for finite elastoplastic strains and isotropic stress response in shells: theoretical and computational analysis. Computer Methods in Applied Mechanics and Engineering 1999; 171(3):243-279.

[8] Kim KD, Liu GZ, Han SC. A resultant 8-node solid-shell element for geometrically nonlinear analysis. Computational Mechanics 2005; 35(5):315-331.

[9] Alves de Sousa RJ, Cardoso, RP, Fontes Valente RA, Yoon JW, Grácio JJ, Natal Jorge RM. A new one - point quadrature enhanced assumed strain (EAS) solid - shell element with multiple integration points along thickness-part II: nonlinear applications. International Journal of Numerical Methods in Engineering 2006; 67(2):160-188.

[10] Battini JM, Pacoste C. On the choice of local element frame for corotational triangular shell elements. Communications in Numerical Methods in Engineering 2004; 20(10):819-825.

[11] Kebari H, Cassell AC. A stabilized 9-node non-linear shell element. International Journal for Numerical Methods in Engineering 1992; 35(1): 37-61.

[12] Kim KD, Lomboy GR. A co-rotational quasi-conforming 4-node resultant shell element for large deformation elasto-plastic analysis. Computer Methods in Applied Mechanics and Engineering 2006; 195(44):6502-6522.

[13] Kim KD, Han SC, Suthasupradit S. Geometrically non-linear analysis of laminated composite structures using a 4-node co-rotational shell element with enhanced strains. International Journal of Non-Linear Mechanics 2007; 42(6):864-881.

[14] Kim KD, Lomboy GR, Han SC. A co-rotational 8-node assumed strain shell element for postbuckling analysis of laminated composite plates and shells. Computational Mechanics 2003; 30(4): 330-342.

[15] Crisfield MA, Moita GF. A co-rotational formulation for 2-D continua including incompatible modes. International Journal for Numerical Methods in Engineering 1996; 39:2619-2633. 
[16] Rankin CC. On choice of best possible corotational element frame. Modeling and Simulation Based Engineering 1988; 1:772-777.

[17] Eriksson A, Pacoste C. Element formulation and numerical techniques for stability problems in shells. Computer Methods in Applied Mechanics and Engineering 2002; 191(35):3775-3810.

[18] Izzuddin BA. Enhanced co-rotational approach for large displacement analysis of plates. International Journal for Numerical Methods in Engineering 2005; 64(10):1350-1374.

[19] Li ZX, Izzuddin BA, and Vu-Quoc L. A 9-node co-rotational quadrilateral shell element. Computational Mechanics 2008; 42(6): 873-884.

[20] Li ZX, Liu YF, Izzuddin BA, Vu-Quoc L. A stabilized co-rotational curved quadrilateral composite shell element. International Journal for Numerical Methods in Engineering 2011; 86(8):975-999.

[21] Li ZX, Zhuo X, Vu-Quoc L, Izzuddin BA, Wei HY. A four-node corotational quadrilateral elastoplastic shell element using vectorial rotational variables. International Journal for Numerical Methods in Engineering 2013; 95(3):181-211.

[22] Zienkiewicz OC, Xu Z, Zeng LF, Samuelsson A, Wiberg NE. Linked interpolation for Reissner-Mindlin plate elements: Part I-A simple quadrilateral, International journal for Numerical Methods in Engineering 1993; 36(18):3043-3056.

[23] Hughes TJR, Tezduyar TE. Finite elements based upon Mindlin plate theory with particular reference to the four-node bilinear isoparametric element. Journal of Applied Mechanics 1981; 48(3):587-596.

[24] Batoz JL, Tahar MB. Evaluation of a new quadrilateral thin plate bending element. International Journal for Numerical Methods in Engineering 1982; 18(11):1655-1677.

[25] Bathe KJ, Lee PS, Hiller JF. Towards improving the MITC9 shell element. Computers \& Structures 2003; 81(8):477-489.

[26] Lee PS, Bathe KJ. Development of MITC isotropic triangular shell finite elements. Computers \& Structures 2004; 82 (11):945-962.

[27] Wisniewski K, Panasz P. Two improvements in formulation of nine-node element MITC9. International Journal for Numerical Methods in Engineering 2013; 93(6):612634.

[28] Kim DN, Bathe KJ. A triangular six-node shell element. Computers \& Structures 2009; 87(23): 1451-1460.

[29] Izzuddin BA. Conceptual issues in geometrically nonlinear analysis of 3D framed structures. Computer Methods in Applied Mechanics and Engineering 2001; 191(8): 1029-1053.

[30] Izzuddin BA. Nonlinear dynamic analysis of framed structures. PhD Thesis, Department of Civil Engineering, Imperial College, University of London, 1991.

[31] Acriniega RA, Reddy JN. Tensor-based finite element formulation for geometrically nonlinear analysis of shell structures. Computer Methods in Applied Mechanics and Engineering 2007; 196(4):1048-1073.

[32] Sze KY, Liu XH, Lo SH. Popular benchmark problems for geometric nonlinear analysis of shells. Finite Elements in Analysis and Design 2004; 40(11):1151-1569. 
[33] Betsch P, Stein E. Numerical implementation of multiplicative elasto-plasticity into assumed strain elements with application to shells at large strains. Computer Methods in Applied Mechanics and Engineering 1999; 179(3): 215-245.

\section{APPENDIX A: LINKED SHAPE FUNCTIONS}

Explicit expressions of $\mathrm{N}_{\mathrm{w}, \mathrm{i}}^{\alpha}, \mathrm{N}_{\theta_{\mathrm{x}}, \mathrm{i}}^{\alpha}$, and $\mathrm{N}_{\theta_{\mathrm{y}}, \mathrm{i}}^{\alpha}\left(\alpha=\mathrm{w}, \theta_{\mathrm{x}}, \theta_{\mathrm{y}}, \gamma\right)$ for the triangular and the quadrilateral elements are given by:

$$
\begin{aligned}
& \mathrm{N}_{\mathrm{w}, \mathrm{i}}^{\mathrm{w}}=\mathrm{N}_{\mathrm{i}}^{\mathrm{L}}+\frac{2}{27} \lambda_{\mathrm{i}}\left(\mathrm{N}_{\mathrm{i}}^{\mathrm{C} 1}-\mathrm{N}_{\mathrm{i}}^{\mathrm{C} 2}\right)-\frac{2}{27} \lambda_{\mathrm{i}_{-}}\left(\mathrm{N}_{\mathrm{i}_{-}}^{\mathrm{C} 1}-\mathrm{N}_{\mathrm{i}_{-}}^{\mathrm{C} 2}\right) \\
& \mathrm{N}_{\mathrm{w}, \mathrm{i}}^{\theta_{\mathrm{x}}}=\frac{2}{27} \lambda_{\mathrm{i}} \mathrm{c}_{\mathrm{i}_{\mathrm{i}}} \mathrm{l}_{(}\left(2 \mathrm{~N}_{\mathrm{i}}^{\mathrm{Cl}}+\mathrm{N}_{\mathrm{i}}^{\mathrm{C} 2}\right)-\frac{2}{27} \lambda_{\mathrm{i}_{-}} \mathrm{c}_{\mathrm{i}_{-}} \mathrm{l}_{\mathrm{i}_{-}}\left(\mathrm{N}_{\mathrm{i}_{-}}^{\mathrm{Cl}}+2 \mathrm{~N}_{\mathrm{i}_{-}}^{\mathrm{C} 2}\right) \\
& \mathrm{N}_{\mathrm{w}, \mathrm{i}}^{\theta_{\mathrm{y}}}=\frac{2}{27} \lambda_{\mathrm{i}_{1}} \mathrm{~s}_{\mathrm{i}} \mathrm{i}_{\mathrm{i}}\left(2 \mathrm{~N}_{\mathrm{i}}^{\mathrm{Cl}}+\mathrm{N}_{\mathrm{i}}^{\mathrm{C} 2}\right)-\frac{2}{27} \lambda_{\mathrm{i}_{-}} \mathrm{s}_{\mathrm{i}_{-}} \mathrm{l}_{\mathrm{i}_{-}}\left(\mathrm{N}_{\mathrm{i}_{-}}^{\mathrm{Cl}}+2 \mathrm{~N}_{\mathrm{i}_{-}}^{\mathrm{C} 2}\right) \\
& \mathrm{N}_{\mathrm{w}, \mathrm{i}}^{\gamma}=\frac{2}{27} \lambda_{\mathrm{i}} \mathrm{l}_{\mathrm{i}}\left(\mathrm{N}_{\mathrm{i}}^{\mathrm{Cl}}-\mathrm{N}_{\mathrm{i}}^{\mathrm{C} 2}\right) \\
& \mathrm{N}_{\theta_{x}, \mathrm{i}}^{\mathrm{w}}=-\frac{3}{2 \mathrm{l}_{\mathrm{i}}} \lambda_{\mathrm{i}} \mathrm{c}_{\mathrm{i}} \mathrm{N}_{\mathrm{i}}^{\mathrm{Q}}+\frac{3}{2 \mathrm{l}_{\mathrm{i}_{-}}} \lambda_{\mathrm{i}_{-}} \mathrm{c}_{\mathrm{i}_{-}} \mathrm{N}_{\mathrm{i}_{-}}^{\mathrm{Q}} \\
& \mathrm{N}_{\theta_{\mathrm{x}}, \mathrm{i}}^{\theta_{\mathrm{x}}}=\mathrm{N}_{\mathrm{i}}^{\mathrm{L}}-\frac{3}{4} \lambda_{\mathrm{i}} \mathrm{c}_{\mathrm{i}}^{2} \mathrm{~N}_{\mathrm{i}}^{\mathrm{Q}}-\frac{3}{4} \lambda_{\mathrm{i}_{-}} \mathrm{c}_{\mathrm{i}_{-}}^{2} \mathrm{~N}_{\mathrm{i}_{-}}^{\mathrm{Q}} \\
& \mathrm{N}_{\theta_{\mathrm{x}}, \mathrm{i}}^{\theta_{\mathrm{y}}}=-\frac{3}{4} \lambda_{\mathrm{i}} \mathrm{c}_{\mathrm{i}} \mathrm{s}_{\mathrm{i}} \mathrm{N}_{\mathrm{i}}^{\mathrm{Q}}-\frac{3}{4} \lambda_{\mathrm{i}_{-}} \mathrm{c}_{\mathrm{i}_{-}} \mathrm{s}_{\mathrm{i}_{-}} \mathrm{N}_{\mathrm{i}_{-}}^{\mathrm{Q}} \\
& \mathrm{N}_{\theta_{x}, \mathrm{i}}^{\gamma}=-\frac{3}{2} \lambda_{\mathrm{i}} \mathrm{c}_{\mathrm{i}} \mathrm{N}_{\mathrm{i}}^{\mathrm{Q}} \\
& \mathrm{N}_{\theta_{\mathrm{y}}, \mathrm{i}}^{\mathrm{w}}=-\frac{3}{2 \mathrm{l}_{\mathrm{i}}} \lambda_{\mathrm{i}_{\mathrm{i}}} \mathrm{s}_{\mathrm{i}} \mathrm{N}_{\mathrm{i}}^{\mathrm{Q}}+\frac{3}{2 \mathrm{l}_{\mathrm{i}_{-}}} \lambda_{\mathrm{i}_{-}} \mathrm{s}_{\mathrm{i}_{-}} \mathrm{N}_{\mathrm{i}_{-}}^{\mathrm{Q}} \\
& \mathrm{N}_{\theta_{\mathrm{y}}, \mathrm{i}}^{\theta_{\mathrm{x}}}=-\frac{3}{4} \lambda_{\mathrm{i}} \mathrm{c}_{\mathrm{i}} \mathrm{s}_{\mathrm{i}} \mathrm{N}_{\mathrm{i}}^{\mathrm{Q}}-\frac{3}{4} \lambda_{\mathrm{i}_{-}} \mathrm{c}_{\mathrm{i}_{-}} \mathrm{s}_{\mathrm{i}_{-}} \mathrm{N}_{\mathrm{i}_{-}}^{\mathrm{Q}} \\
& \mathrm{N}_{\theta_{\mathrm{y}}, \mathrm{i}}^{\theta_{\mathrm{y}}}=\mathrm{N}_{\mathrm{i}}^{\mathrm{L}}-\frac{3}{4} \lambda_{\mathrm{i}} \mathrm{s}_{\mathrm{i}}^{2} \mathrm{~N}_{\mathrm{i}}^{\mathrm{Q}}-\frac{3}{4} \lambda_{\mathrm{i}_{-}} \mathrm{s}_{\mathrm{i}_{-}}^{2} \mathrm{~N}_{\mathrm{i}_{-}}^{\mathrm{Q}} \\
& \mathrm{N}_{\theta_{\mathrm{y}}, \mathrm{i}}^{\gamma}=-\frac{3}{2} \lambda_{\mathrm{i}_{\mathrm{i}}} \mathrm{s}_{\mathrm{i}} \mathrm{N}^{\mathrm{Q}}
\end{aligned}
$$

where the shape functions $\mathrm{N}_{\mathrm{i}}^{\mathrm{L}}, \mathrm{N}_{\mathrm{i}}^{\mathrm{Q}}, \mathrm{N}_{\mathrm{i}}^{\mathrm{C}_{1}}$, and $\mathrm{N}_{\mathrm{i}}^{\mathrm{C}_{2}}$ refer to (23a-b) for the triangular element and to $(24 a-b)$ for the quadrilateral element. 


\section{APPENDIX B: Q MATRIX}

The $\mathbf{Q}$ matrix can be established from (25) and (27), with the non-zero terms given by:

$$
\begin{aligned}
& \mathrm{Q}_{\mathrm{i}, \mathrm{i}}=\frac{-1}{\mathrm{c}_{\mathrm{i}} \mathrm{s}_{\mathrm{i}_{-}}-\mathrm{c}_{\mathrm{i}_{-}} \mathrm{s}_{\mathrm{i}}}\left[\frac{\left(1-\lambda_{\mathrm{i}}\right)}{\mathrm{l}_{\mathrm{i}}} \mathrm{s}_{\mathrm{i}_{-}}+\frac{\left(1-\lambda_{\mathrm{i}_{-}}\right)}{\mathrm{l}_{\mathrm{i}_{-}}} \mathrm{s}_{\mathrm{i}}\right] \\
& \mathrm{Q}_{\mathrm{i}, \mathrm{i}_{-}}=\frac{1}{\mathrm{c}_{\mathrm{i}} \mathrm{s}_{\mathrm{i}_{-}}-\mathrm{c}_{\mathrm{i}_{-}} \mathrm{s}_{\mathrm{i}}}\left[\frac{\left(1-\lambda_{\mathrm{i}_{-}}\right)}{\mathrm{i}_{\mathrm{i}_{-}}} \mathrm{s}_{\mathrm{i}}\right] \\
& \mathrm{Q}_{\mathrm{i}, \mathrm{i}_{+}}=\frac{-1}{\mathrm{c}_{\mathrm{i}} \mathrm{s}_{\mathrm{i}_{-}}-\mathrm{c}_{\mathrm{i}_{-}} \mathrm{s}_{\mathrm{i}}}\left[\frac{\left(1-\lambda_{\mathrm{i}}\right)}{\mathrm{l}_{\mathrm{i}}} \mathrm{s}_{\mathrm{i}_{-}}\right]
\end{aligned}
$$

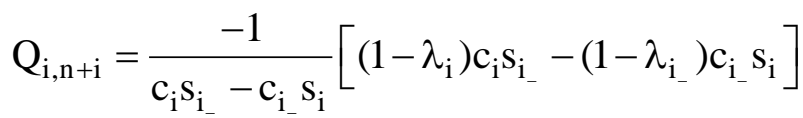

$$
\begin{aligned}
& \mathrm{Q}_{\mathrm{i}, 2 \mathrm{n}+\mathrm{i}}=\frac{\mathrm{s}_{\mathrm{i}} \mathrm{s}_{\mathrm{i}_{-}}}{\mathrm{c}_{\mathrm{i}} \mathrm{s}_{\mathrm{i}_{-}}-\mathrm{c}_{\mathrm{i}_{-}} \mathrm{s}_{\mathrm{i}}}\left[\lambda_{\mathrm{i}}-\lambda_{\mathrm{i}_{-}}\right] \\
& \mathrm{Q}_{\mathrm{i}, 4 \mathrm{n}+\mathrm{i}}=\frac{\lambda_{\mathrm{i}} \mathrm{s}_{\mathrm{i}_{-}}}{\mathrm{c}_{\mathrm{i}} \mathrm{s}_{\mathrm{i}_{-}}-\mathrm{c}_{\mathrm{i}_{-}} \mathrm{s}_{\mathrm{i}}} \\
& \mathrm{Q}_{\mathrm{i}, 4 \mathrm{n}+\mathrm{i}_{-}}=\frac{-\lambda_{\mathrm{i}_{-}} \mathrm{s}_{\mathrm{i}}}{\mathrm{c}_{\mathrm{i}} \mathrm{s}_{\mathrm{i}_{-}}-\mathrm{c}_{\mathrm{i}_{-}} \mathrm{s}_{\mathrm{i}}} \\
& \mathrm{Q}_{\mathrm{n}+\mathrm{i}, \mathrm{i}}=\frac{1}{\mathrm{c}_{\mathrm{i}} \mathrm{s}_{\mathrm{i}_{-}}-\mathrm{c}_{\mathrm{i}_{-}} \mathrm{s}_{\mathrm{i}}}\left[\frac{\left(1-\lambda_{\mathrm{i}}\right)}{\mathrm{l}_{\mathrm{i}}} \mathrm{c}_{\mathrm{i}_{-}}+\frac{\left(1-\lambda_{\mathrm{i}_{-}}\right)}{\mathrm{l}_{\mathrm{i}_{-}}} \mathrm{c}_{\mathrm{i}}\right] \\
& \mathrm{Q}_{\mathrm{n}+\mathrm{i}, \mathrm{i}_{-}}=\frac{-1}{\mathrm{c}_{\mathrm{i}} \mathrm{s}_{\mathrm{i}_{-}}-\mathrm{c}_{\mathrm{i}_{-}} \mathrm{s}_{\mathrm{i}}}\left[\frac{\left(1-\lambda_{\mathrm{i}_{-}}\right)}{\mathrm{l}_{\mathrm{i}_{-}}} \mathrm{c}_{\mathrm{i}}\right] \\
& \mathrm{Q}_{\mathrm{n}+\mathrm{i}, \mathrm{i}_{+}}=\frac{-1}{\mathrm{c}_{\mathrm{i}} \mathrm{s}_{\mathrm{i}_{-}}-\mathrm{c}_{\mathrm{i}_{-}} \mathrm{s}_{\mathrm{i}}}\left[\frac{\left(1-\lambda_{\mathrm{i}}\right)}{\mathrm{l}_{\mathrm{i}}} \mathrm{c}_{\mathrm{i}_{-}}\right] \\
& \mathrm{Q}_{\mathrm{n}+\mathrm{i}, \mathrm{n}+\mathrm{i}}=\frac{-\mathrm{c}_{\mathrm{i}} \mathrm{c}_{\mathrm{i}_{--}}}{\mathrm{c}_{\mathrm{i}} \mathrm{s}_{\mathrm{i}_{-}}-\mathrm{c}_{\mathrm{i}_{-}} \mathrm{s}_{\mathrm{i}}}\left[\lambda_{\mathrm{i}}-\lambda_{\mathrm{i}_{-}}\right] \\
& \mathrm{Q}_{\mathrm{n}+\mathrm{i}, 2 \mathrm{n}+\mathrm{i}}=\frac{1}{\mathrm{c}_{\mathrm{i}} \mathrm{s}_{\mathrm{i}_{-}}-\mathrm{c}_{\mathrm{i}_{-}} \mathrm{s}_{\mathrm{i}}}\left[\left(1-\lambda_{\mathrm{i}}\right) \mathrm{c}_{\mathrm{i}_{-}} \mathrm{s}_{\mathrm{i}}-\left(1-\lambda_{\mathrm{i}_{-}}\right) \mathrm{c}_{\mathrm{i}} \mathrm{s}_{\mathrm{i}_{-}}\right] \\
& \mathrm{Q}_{\mathrm{n}+\mathrm{i}, 4 \mathrm{n}+\mathrm{i}}=\frac{-\lambda_{\mathrm{i}} \mathrm{c}_{\mathrm{i}_{-}}}{\mathrm{c}_{\mathrm{i}} \mathrm{s}_{\mathrm{i}_{-}}-\mathrm{c}_{\mathrm{i}_{-}} \mathrm{s}_{\mathrm{i}}} \\
& \mathrm{Q}_{\mathrm{n}+\mathrm{i}, 4 \mathrm{n}+\mathrm{i}_{-}}=\frac{\lambda_{\mathrm{i}_{-}} \mathrm{c}_{\mathrm{i}}}{\mathrm{c}_{\mathrm{i}_{\mathrm{i}} \mathrm{s}_{-}-\mathrm{c}_{\mathrm{i}_{-}} \mathrm{s}_{\mathrm{i}}}}
\end{aligned}
$$

where $i_{-}=\bmod (i+n-2, n)+1$. 


\section{APPENDIX C: FIRST DERIVATIVES OF LOCAL PARAMETERS}

The first derivatives of local parameters with respect to global parameters involve the differentiation of the local translations and rotations ( $\mathbf{t}$ and $\overline{\mathbf{r}}$ ) with respect to the global translational and rotational parameters ( $\mathbf{d}$ and $\overline{\mathbf{n}}$ ), which can be obtained through chain differentiation.

The required first derivatives for triangular and quadrilateral elements are obtained from (49)-(51) [18]:

$$
\begin{gathered}
\frac{\partial \mathbf{t}_{\mathrm{i}, \mathrm{m}}}{\partial \mathbf{d}_{\mathrm{k}, \mathrm{p}}}=\delta_{\mathrm{ik}} \mathbf{R}_{\mathrm{m}}\left\{\begin{array}{c}
\delta_{1 \mathrm{p}} \\
\delta_{2 \mathrm{p}} \\
\delta_{3 \mathrm{p}}
\end{array}\right\}+\frac{\partial \mathbf{R}_{\mathrm{m}}}{\partial \mathbf{d}_{\mathrm{k}, \mathrm{p}}}\left(\mathbf{d}_{\mathrm{i}}+\mathbf{v}_{\mathrm{io}}^{\mathrm{o}}\right) \quad(\mathrm{i} \text { and } \mathrm{k}=1 \rightarrow \mathrm{n}, \mathrm{m} \text { and } \mathrm{p}=1 \rightarrow 3) \\
\frac{\partial \mathbf{t}_{\mathrm{i}, \mathrm{m}}}{\partial \overline{\mathbf{n}}_{\mathrm{k}, \mathrm{p}}}=0 \quad(\mathrm{i} \text { and } \mathrm{k}=1 \rightarrow \mathrm{n}, \mathrm{m}=1 \rightarrow 3, \mathrm{p}=1 \rightarrow 2) \\
\frac{\partial \overline{\mathbf{r}}_{\mathrm{i}, \mathrm{m}}}{\partial \mathbf{d}_{\mathrm{k}, \mathrm{p}}}=-\frac{\partial \overline{\mathbf{R}}_{\mathrm{m}}}{\partial \mathbf{d}_{\mathrm{k}, \mathrm{p}}} \mathbf{n}_{\mathrm{i}} \quad(\mathrm{i} \text { and } \mathrm{k}=1 \rightarrow \mathrm{n}, \mathrm{m}=1 \rightarrow 2, \mathrm{p}=1 \rightarrow 3) \\
\frac{\partial \overline{\mathbf{r}}_{\mathrm{i}, \mathrm{m}}}{\partial \overline{\mathbf{n}}_{\mathrm{k}, \mathrm{p}}}=-\overline{\mathbf{R}}_{\mathrm{m}} \frac{\partial \mathbf{n}_{\mathrm{i}}}{\partial \overline{\mathbf{n}}_{\mathrm{k}, \mathrm{p}}} \quad(\mathrm{i} \text { and } \mathrm{k}=1 \rightarrow \mathrm{n}, \mathrm{m} \text { and } \mathrm{p}=1 \rightarrow 2)
\end{gathered}
$$

The main difference in the first derivatives between the bisector and zero-macrospin definitions of the co-rotational system arises from the partial derivatives of $\left(\mathbf{c}_{\mathrm{x}}, \mathbf{c}_{\mathrm{y}}, \mathbf{c}_{\mathrm{z}}\right)$ with respect to global translational degrees of freedom. The first derivatives of $\left(\mathbf{c}_{\mathrm{x}}, \mathbf{c}_{\mathrm{y}}, \mathbf{c}_{\mathrm{z}}\right)$ with respect to global translational degrees of freedom in accordance with the bisector definition have been provided in [18]; hereafter, the first derivatives associated with the zero-macrospin definition are obtained.

For quadrilateral elements:

$$
\begin{array}{r}
\frac{\partial \mathbf{c}_{\mathrm{x}}}{\partial \mathbf{d}_{\mathrm{i}, \mathrm{p}}}=\frac{\mathbf{I}-\mathbf{c}_{\mathrm{x}} \mathbf{c}_{\mathrm{x}}^{\mathrm{T}}}{\left|\mathbf{c}_{\mathrm{x}}^{\prime}+\mathbf{c}_{\mathrm{y}}^{\prime \mathrm{n}}\right|}\left(\frac{\partial \mathbf{c}_{\mathrm{x}}^{\prime}}{\partial \mathbf{d}_{\mathrm{i}, \mathrm{p}}}+\frac{\partial \mathbf{c}_{\mathrm{y}}^{\mathrm{n}}}{\partial \mathbf{d}_{\mathrm{i}, \mathrm{p}}}\right) \\
\frac{\partial \mathbf{c}_{\mathrm{x}}^{\prime}}{\partial \mathbf{d}_{\mathrm{i}, \mathrm{p}}}=\left[\mathrm{a}_{\mathrm{x} 1}\left(\delta_{3 \mathrm{i}}-\delta_{1 \mathrm{i}}\right)+\mathrm{a}_{\mathrm{x} 2}\left(\delta_{4 \mathrm{i}}-\delta_{2 \mathrm{i}}\right)\right]\left\{\begin{array}{c}
\delta_{1 \mathrm{p}} \\
\delta_{2 \mathrm{p}} \\
\delta_{3 \mathrm{p}}
\end{array}\right\}
\end{array}
$$




$$
\begin{gathered}
\frac{\partial \mathbf{c}_{\mathrm{y}}^{\prime \mathrm{n}}}{\partial \mathbf{d}_{\mathrm{i}, \mathrm{p}}}=\frac{\partial \mathbf{c}_{\mathrm{y}}^{\prime}}{\partial \mathbf{d}_{\mathrm{i}, \mathrm{p}}} \times \mathbf{c}_{\mathrm{z}}+\mathbf{c}_{\mathrm{y}}^{\prime} \times \frac{\partial \mathbf{c}_{\mathrm{z}}}{\partial \mathbf{d}_{\mathrm{i}, \mathrm{p}}} \\
\frac{\partial \mathbf{c}_{\mathrm{y}}^{\prime}}{\partial \mathbf{d}_{\mathrm{i}, \mathrm{p}}}=\left[\mathrm{a}_{\mathrm{y} 1}\left(\delta_{3 \mathrm{i}}-\delta_{1 \mathrm{i}}\right)+\mathrm{a}_{\mathrm{y} 2}\left(\delta_{4 \mathrm{i}}-\delta_{2 \mathrm{i}}\right)\right]\left\{\begin{array}{l}
\delta_{1 \mathrm{p}} \\
\delta_{2 \mathrm{p}} \\
\delta_{3 \mathrm{p}}
\end{array}\right\} \\
\frac{\partial \mathbf{c}_{\mathrm{z}}}{\partial \mathbf{d}_{\mathrm{i}, \mathrm{p}}}=\frac{\mathbf{I}-\mathbf{c}_{\mathrm{z}} \mathbf{c}_{\mathrm{z}}^{\mathrm{T}}}{\left|\mathbf{v}_{13} \times \mathbf{v}_{24}\right|}\left[\begin{array}{l}
\left.\left(\delta_{3 \mathrm{i}}-\delta_{1 \mathrm{i}}\right)\left\{\begin{array}{l}
\delta_{1 \mathrm{p}} \\
\delta_{2 \mathrm{p}} \\
\delta_{3 \mathrm{p}}
\end{array}\right\} \times \mathbf{v}_{24}+\left(\delta_{4 \mathrm{i}}-\delta_{2 \mathrm{i}}\right) \mathbf{v}_{13} \times\left\{\begin{array}{l}
\delta_{1 \mathrm{p}} \\
\delta_{2 \mathrm{p}} \\
\delta_{3 \mathrm{p}}
\end{array}\right\}\right] \\
\frac{\partial \mathbf{c}_{\mathrm{y}}}{\partial \mathbf{d}_{\mathrm{i}, \mathrm{p}}}=\frac{\partial \mathbf{c}_{\mathrm{z}}}{\partial \mathbf{d}_{\mathrm{i}, \mathrm{p}}} \times \mathbf{c}_{\mathrm{x}}+\mathbf{c}_{\mathrm{z}} \times \frac{\partial \mathbf{c}_{\mathrm{x}}}{\partial \mathbf{d}_{\mathrm{i}, \mathrm{p}}}
\end{array}\right.
\end{gathered}
$$

The expressions in (C5), (C7) and (C10) are also applicable to triangular elements, while the derivatives $\partial \mathbf{c}_{\mathrm{x}}^{\prime} / \partial \mathbf{d}_{\mathrm{i}, \mathrm{p}}, \partial \mathbf{c}_{\mathrm{y}}^{\prime} / \partial \mathbf{d}_{\mathrm{i}, \mathrm{p}}$, and $\partial \mathbf{c}_{\mathrm{z}} / \partial \mathbf{d}_{\mathrm{i}, \mathrm{p}}$ are different:

$$
\begin{gathered}
\frac{\partial \mathbf{c}_{\mathrm{x}}^{\prime}}{\partial \mathbf{d}_{\mathrm{i}, \mathrm{p}}}=\mathrm{a}_{\mathrm{x} 1}\left(\delta_{2 \mathrm{i}}-\delta_{1 \mathrm{i}}\right)\left\{\begin{array}{l}
\delta_{1 \mathrm{p}} \\
\delta_{2 \mathrm{p}} \\
\delta_{3 \mathrm{p}}
\end{array}\right\} \\
\frac{\partial \mathbf{c}_{\mathrm{y}}^{\prime}}{\partial \mathbf{d}_{\mathrm{i}, \mathrm{p}}}=\left[\mathrm{a}_{\mathrm{y} 1}\left(\delta_{2 \mathrm{i}}-\delta_{1 \mathrm{i}}\right)+\mathrm{a}_{\mathrm{y} 2}\left(\delta_{3 \mathrm{i}}-\delta_{2 \mathrm{i}}\right)\right]\left\{\begin{array}{l}
\delta_{1 \mathrm{p}} \\
\delta_{2 \mathrm{p}} \\
\delta_{3 \mathrm{p}}
\end{array}\right\} \\
\frac{\partial \mathbf{c}_{\mathrm{z}}}{\partial \mathbf{d}_{\mathrm{i}, \mathrm{p}}}=\frac{\mathbf{I}-\mathbf{c}_{\mathrm{z}} \mathbf{c}_{\mathrm{z}}^{\mathrm{T}}}{\left|\mathbf{v}_{12} \times \mathbf{v}_{23}\right|}\left[\left(\delta_{2 \mathrm{i}}-\delta_{1 \mathrm{i}}\right)\left\{\begin{array}{l}
\delta_{1 \mathrm{p}} \\
\delta_{2 \mathrm{p}} \\
\delta_{3 \mathrm{p}}
\end{array}\right\} \times \mathbf{v}_{23}+\left(\delta_{3 \mathrm{i}}-\delta_{2 \mathrm{i}}\right) \mathbf{v}_{12} \times\left\{\begin{array}{l}
\delta_{1 \mathrm{p}} \\
\delta_{2 \mathrm{p}} \\
\delta_{3 \mathrm{p}}
\end{array}\right\}\right]
\end{gathered}
$$


Table 1: Variants of co-rotational system definitions.

\begin{tabular}{|c|c|c|}
\hline Element Code & Co-rotational system & Local Element Formulation \\
\hline ZMQ & Zero-macrospin & Quadrilateral / Flat \\
\hline BSQ & Bisector & Quadrilateral / Flat \\
\hline FEQ & First-edge & Quadrilateral / Flat \\
\hline ZMT & Zero-macrospin & Triangular / Flat \\
\hline BST & Bisector & Triangular / Flat \\
\hline FET & First-edge & Triangular / Flat \\
\hline BSQ2 & Bisector & Quadrilateral / Curved \\
\hline ZMT2 & Zero-macrospin & Triangular / Curved \\
\hline
\end{tabular}


Table 2: Number of degrees of freedom in different models.

\begin{tabular}{|c|c|c|c|c|}
\hline Model & Mesh & Number of nodes/element & Element DOFs & Total active DOFs \\
\hline Present & $24 \times 4$ & 4 & $5 /$ node $+4 /$ edge & 1464 \\
\hline Arciniega \& Reddy [31] & $5 \times 1$ & 49 & $7 /$ node & 1715 \\
\hline Sze et al. [32] & $30 \times 6$ & 4 & $6 /$ node & 1260 \\
\hline
\end{tabular}


Table 3: Deflections at pinched points A and B under $\mathrm{F}=0.03$.

\begin{tabular}{|c|c|c|}
\hline \multirow{2}{*}{ Model } & \multicolumn{2}{|c|}{ Deflection } \\
\cline { 2 - 3 } & Point A & Point B \\
\hline BSQ2-1 & 4.0452 & 8.3230 \\
\hline BSQ2-2 & 4.0452 & 8.3230 \\
\hline ZMT2-1 & 4.0278 & 8.2993 \\
\hline ZMT2-2 & 4.0278 & 8.2993 \\
\hline MH6T-1 (Li et al. [4]) & 3.8313 & 7.9992 \\
\hline MH6T-2 (Li et al. [4]) & 3.8278 & 7.9864 \\
\hline
\end{tabular}



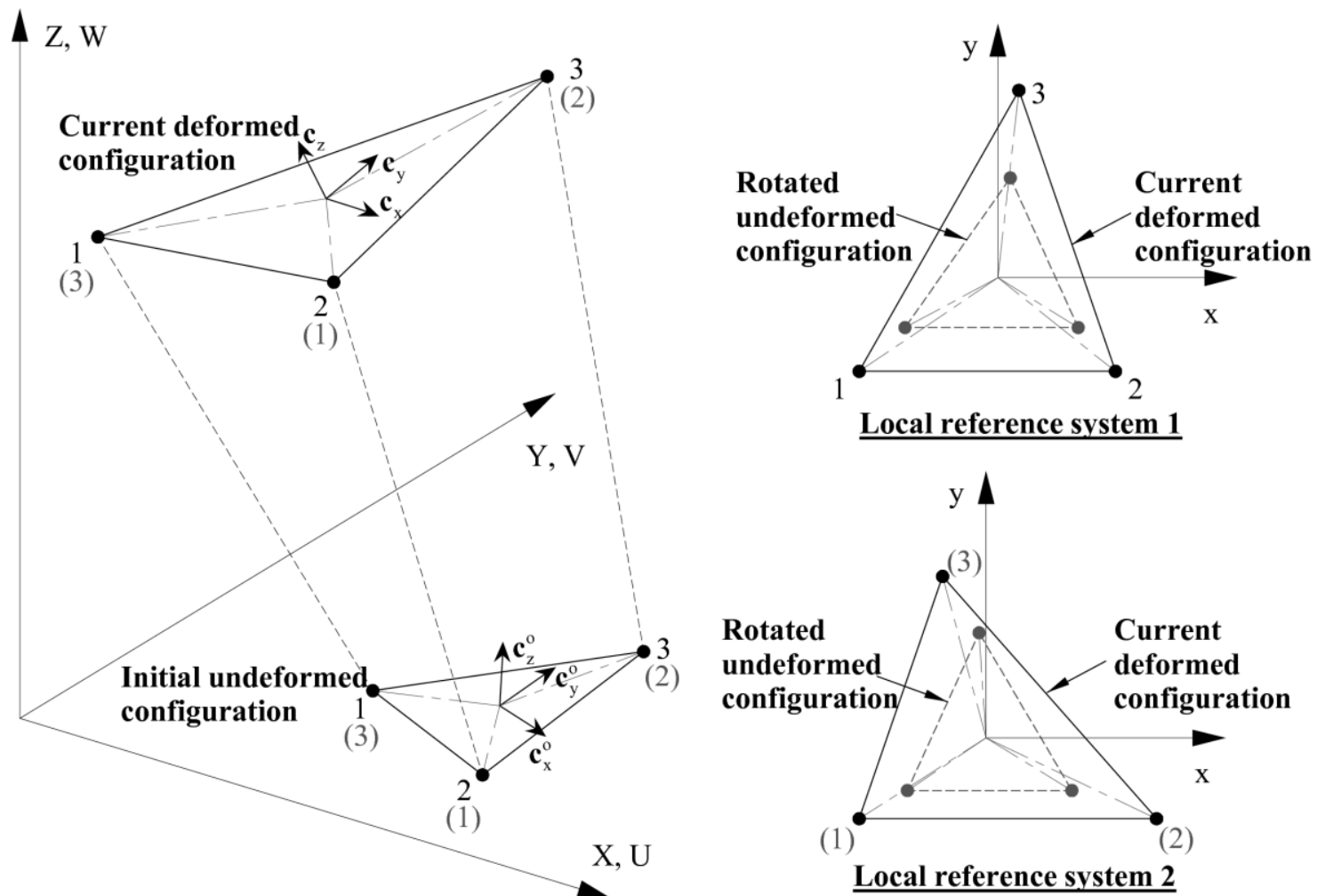

Figure 1: First-edge co-rotational system and local deformations for triangular element. 

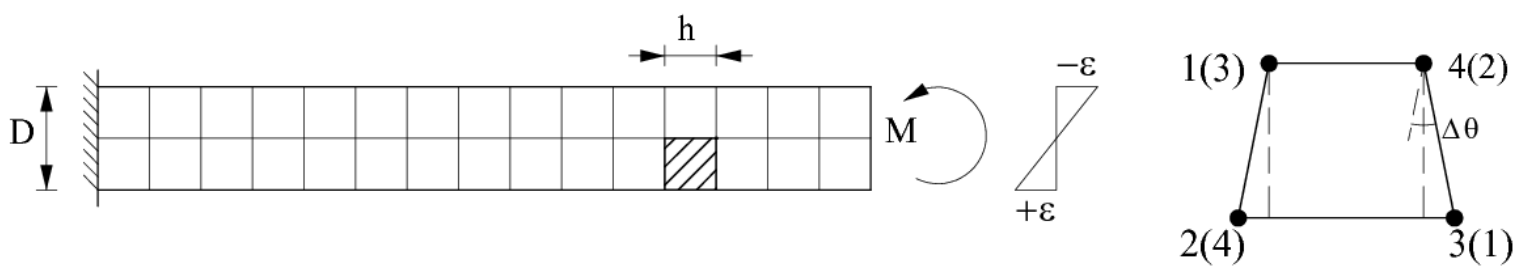

a. Rectangular plate subject to planar curvature
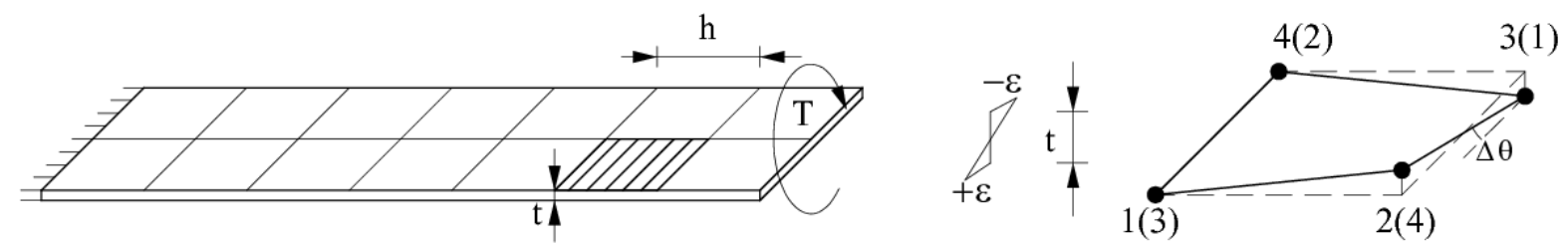

b. Rectangular plate subject to out-of-plane twisting curvature

Figure 2: Rectangular plate subject to planar and out-of-plane twisting curvatures. 


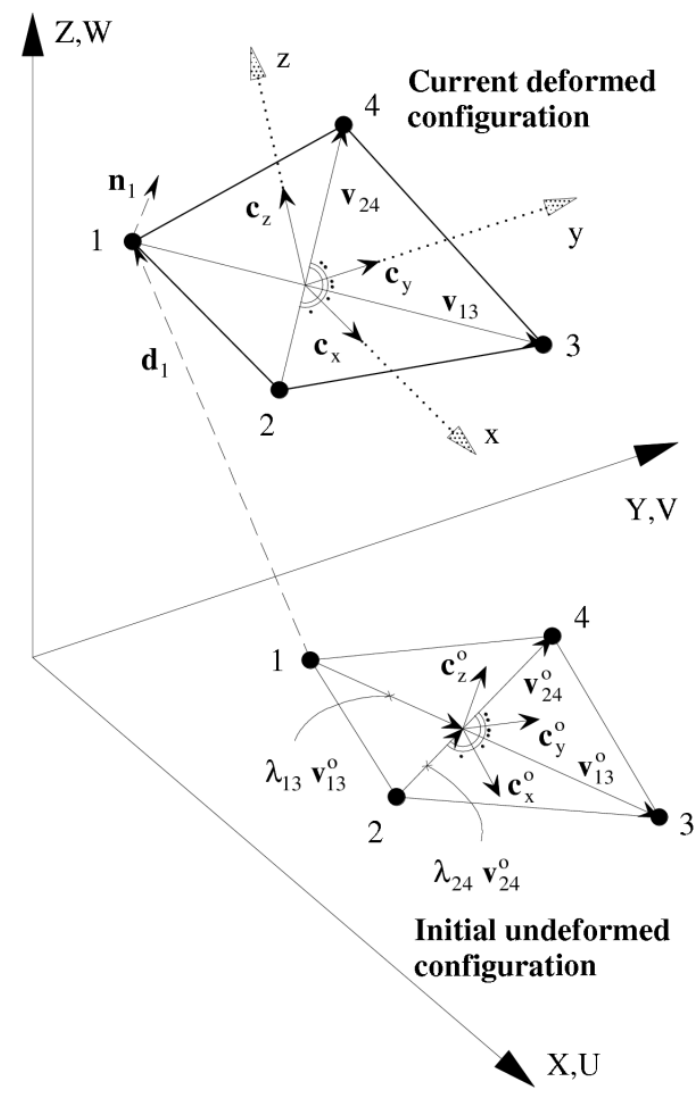

$\begin{array}{ll}\text { Rotated } & \text { Current } \\ \text { undeformed } & \text { deformed }\end{array}$

configuration y $\Lambda$ configuration

4

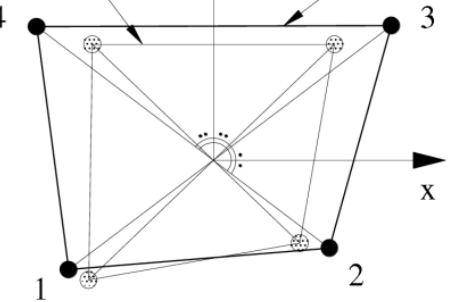

Local reference system

Figure 3: Bisector local co-rotational system and global nodal freedoms for quadrilateral element. 


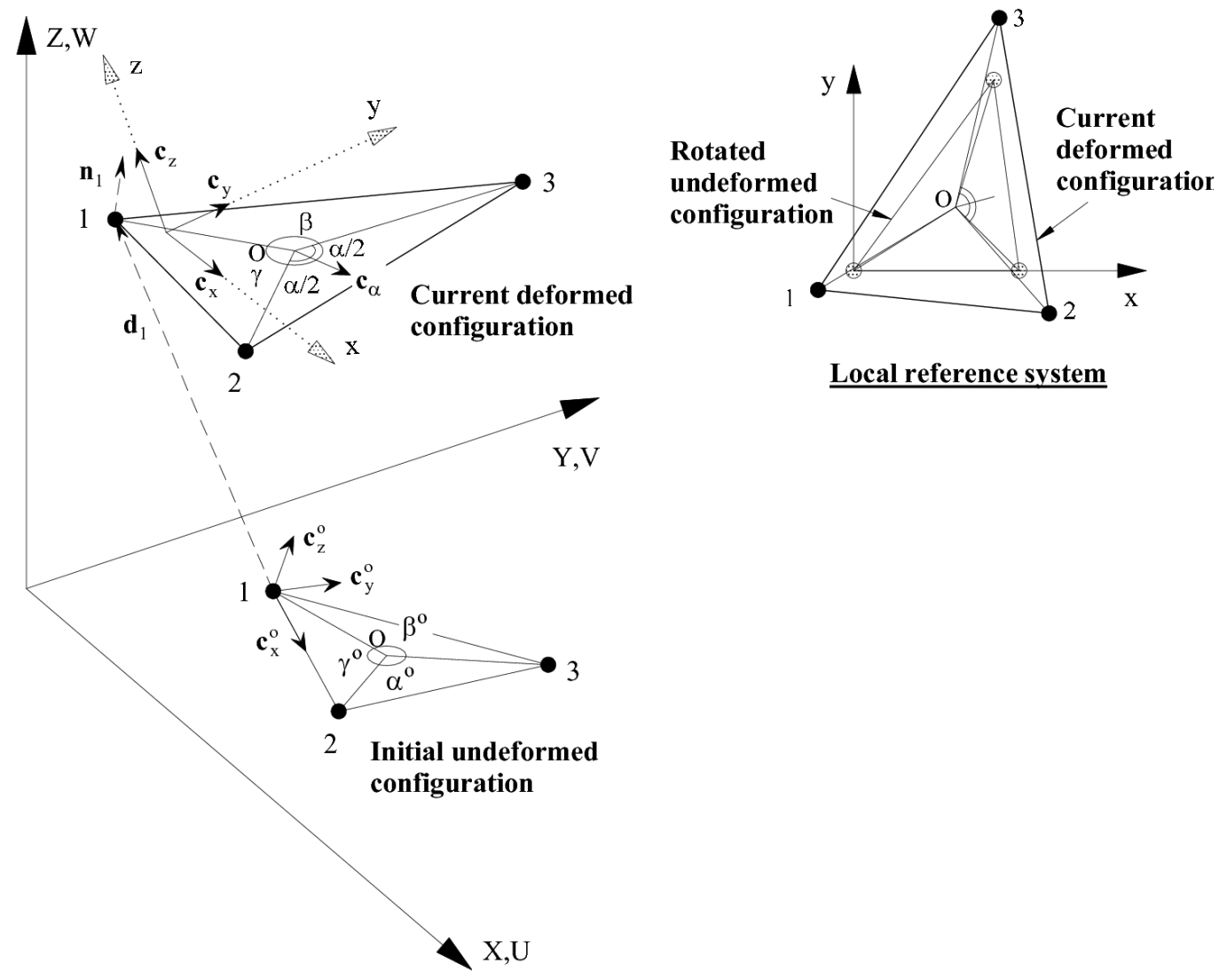

Figure 4: Bisector local co-rotational system and global nodal freedoms for triangular element. 

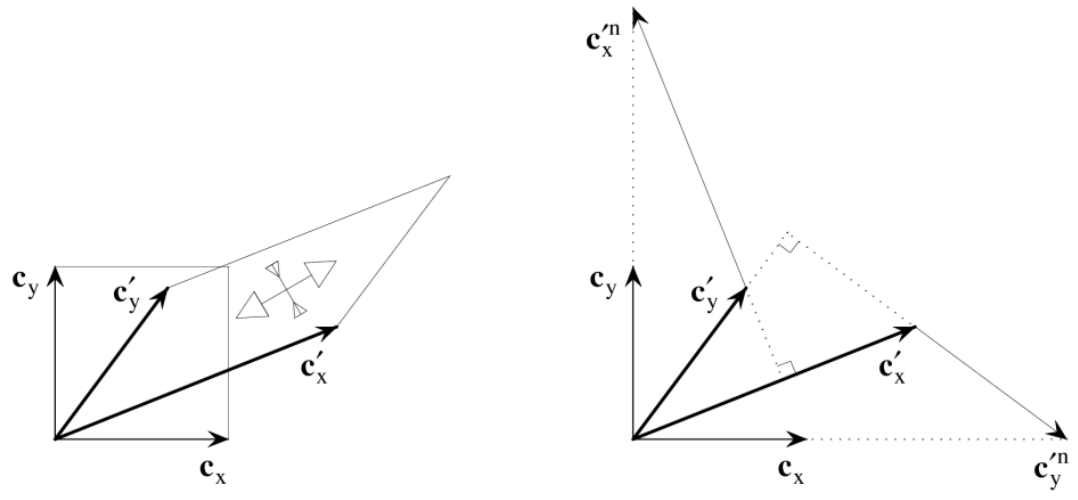

Figure 5: Influence of a uniform 'stretch' operation on a unit square area. 


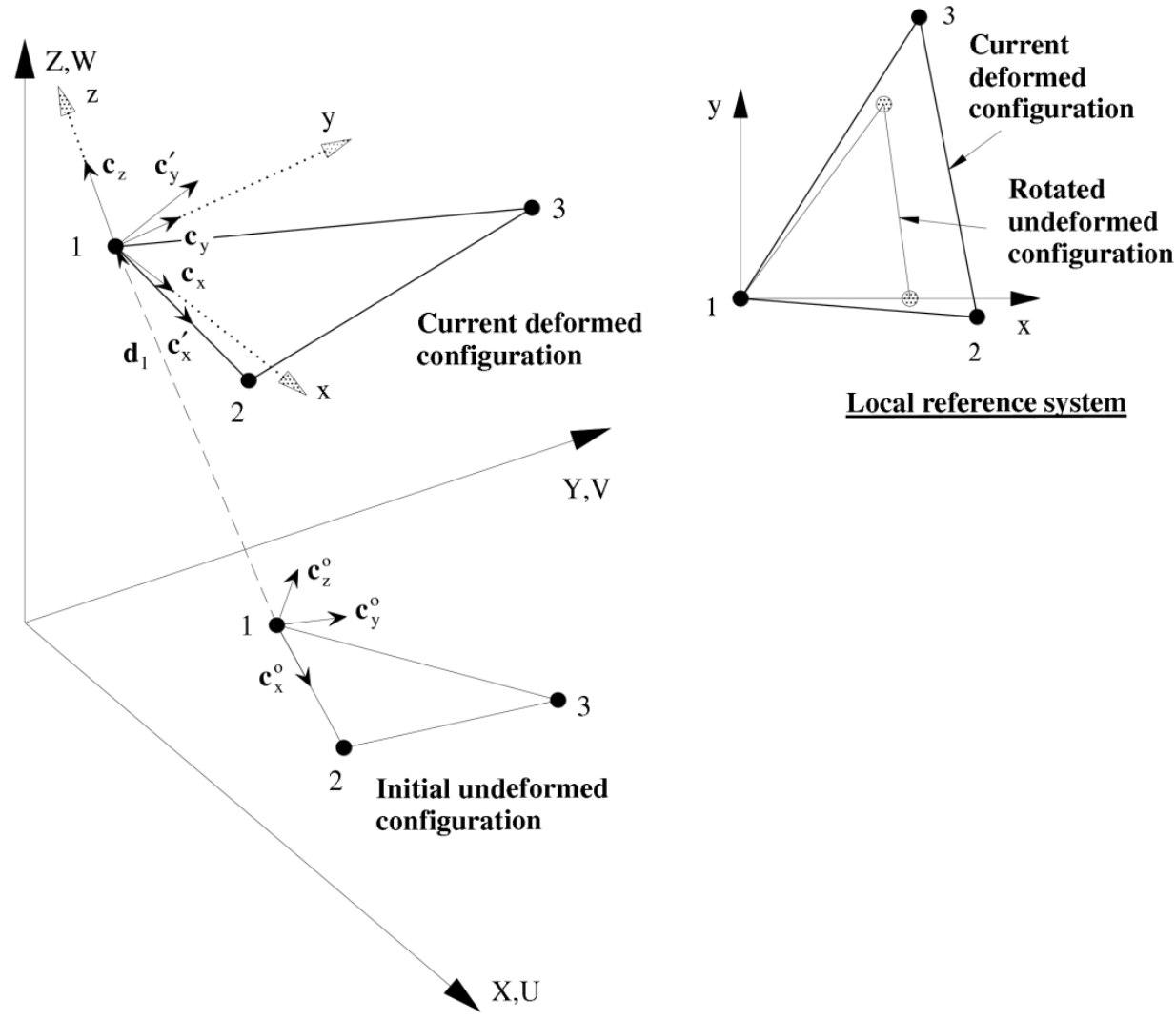

Figure 6: Zero-macrospin local co-rotational system for triangular element. 

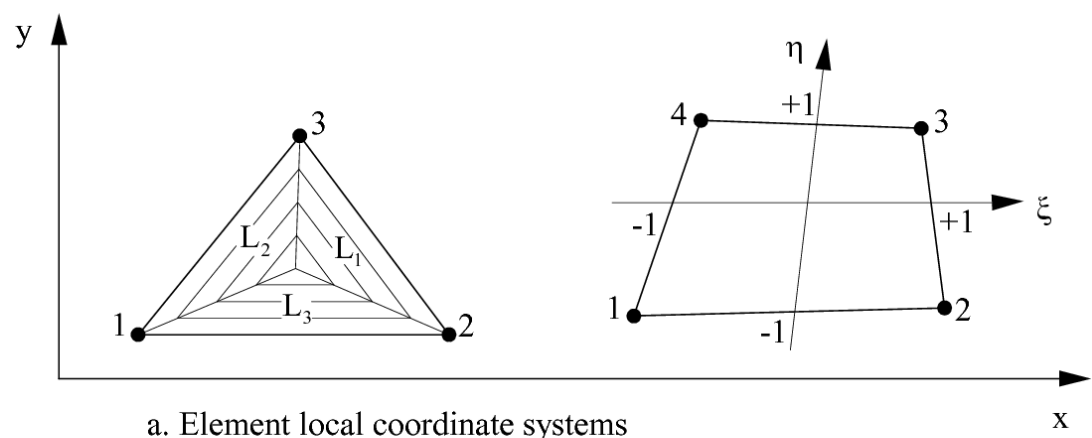

a. Element local coordinate systems
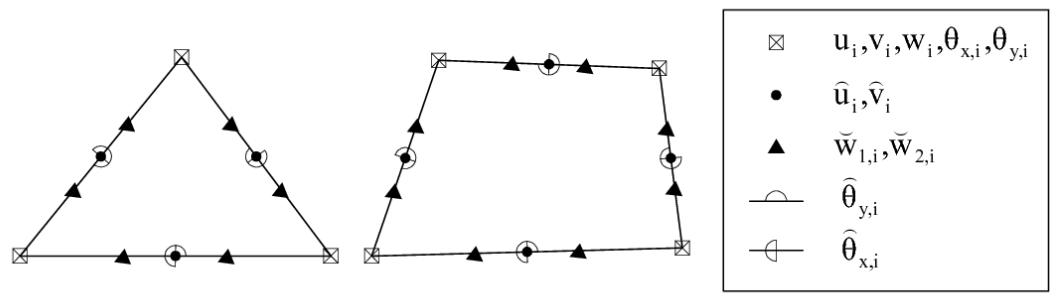

b. Element parameters before application of edge shear constraints
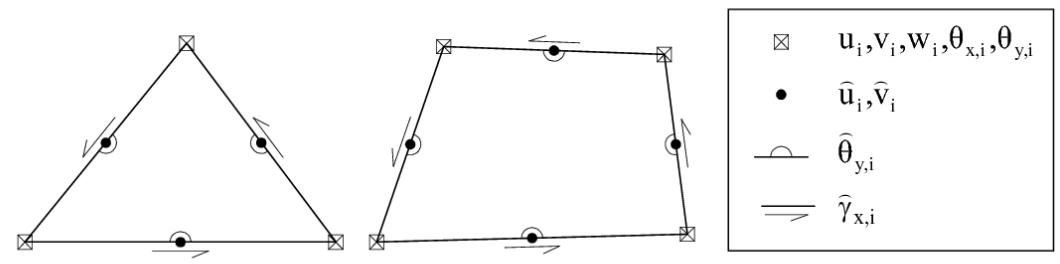

c. Element parameters after application of edge shear constraints

Figure 7: Reference system and parameters of quadrilateral and triangular elements. 


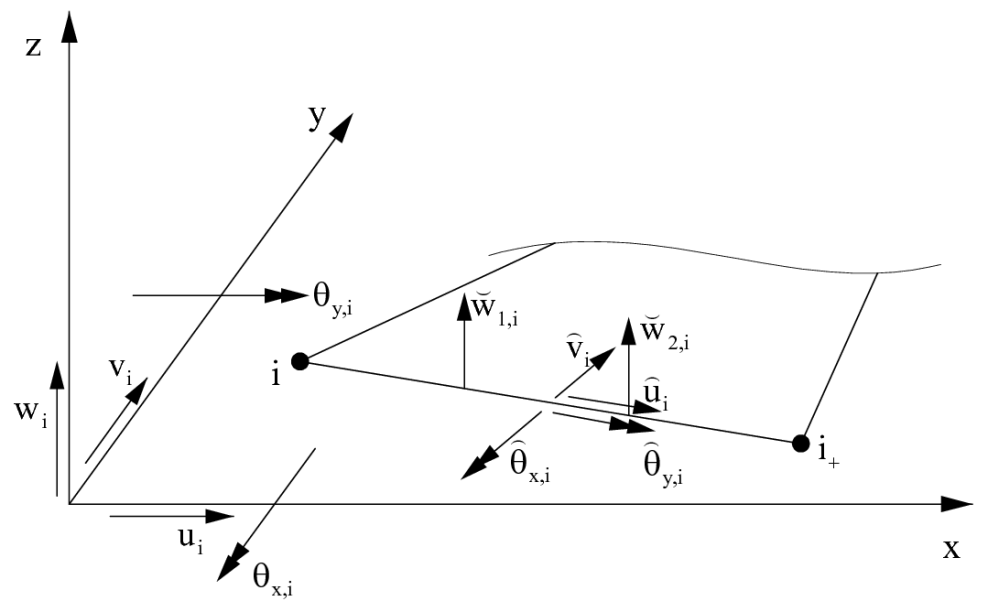

Figure 8: Sign convention for nodal and hierarchic edge parameters. 

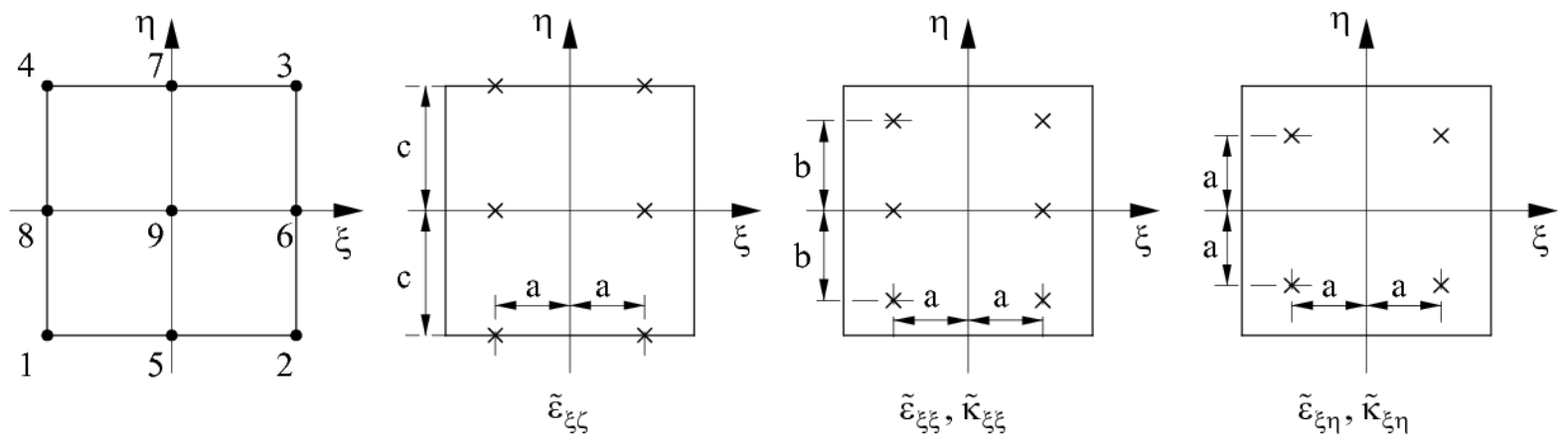

Figure 9: Positions of tying points for MITC9 element $(\mathrm{a}=1 / \sqrt{3}, \mathrm{~b}=\sqrt{3 / 5}$, and $\mathrm{c}=1)$. 

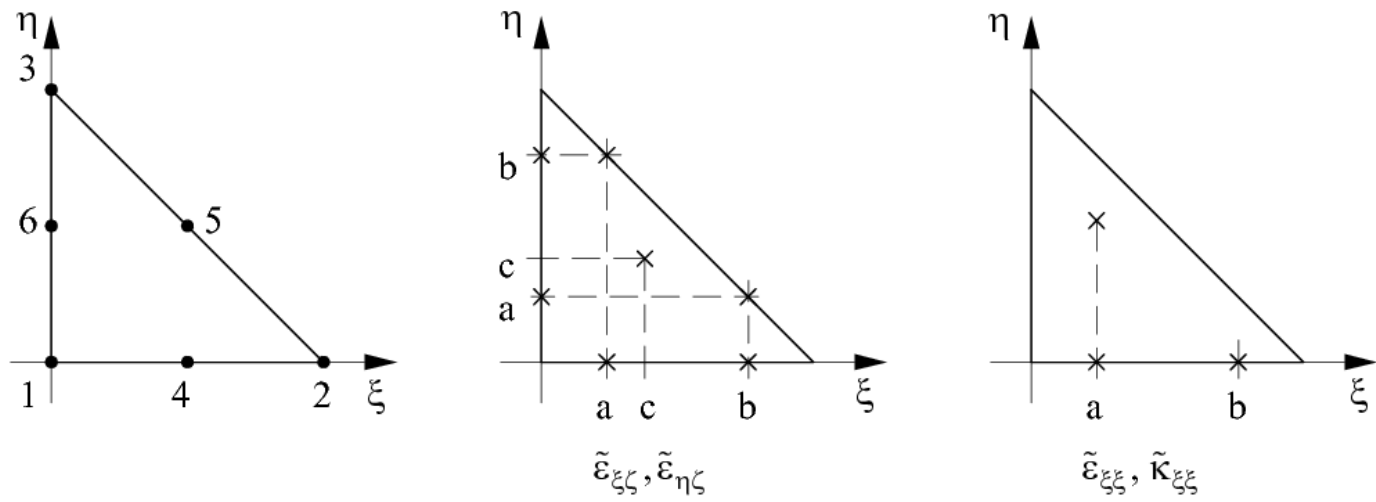

Figure 10: Positions of tying points for MITC6 element $\left(\mathrm{a}=\frac{1}{2}-\frac{1}{2 \sqrt{3}}, \mathrm{~b}=\frac{1}{2}+\frac{1}{2 \sqrt{3}}\right.$, and $\mathrm{c}=\frac{1}{3}$ ). 

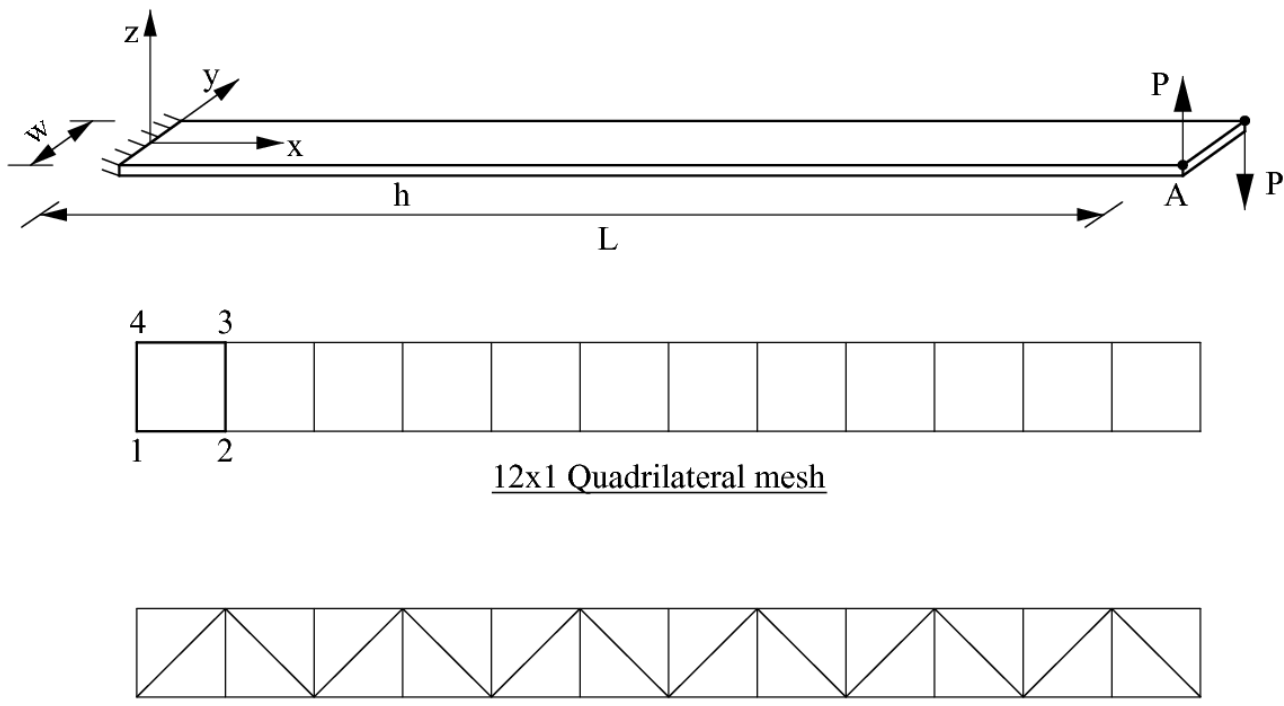

12x1 Triangular mesh

Figure 11: Clamped strip under end torsion. 


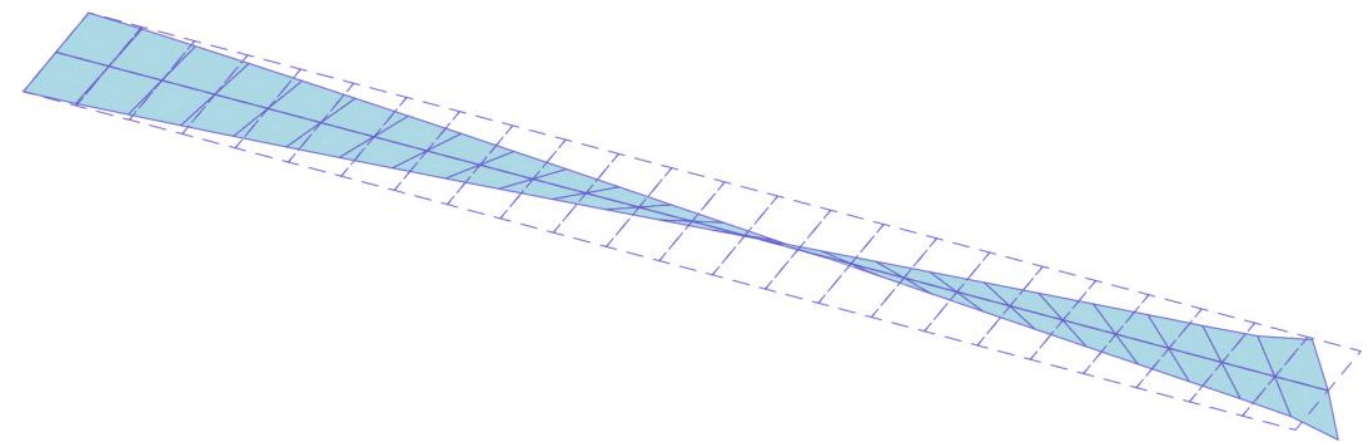

Figure 12: Deformed configuration of the clamped strip. 


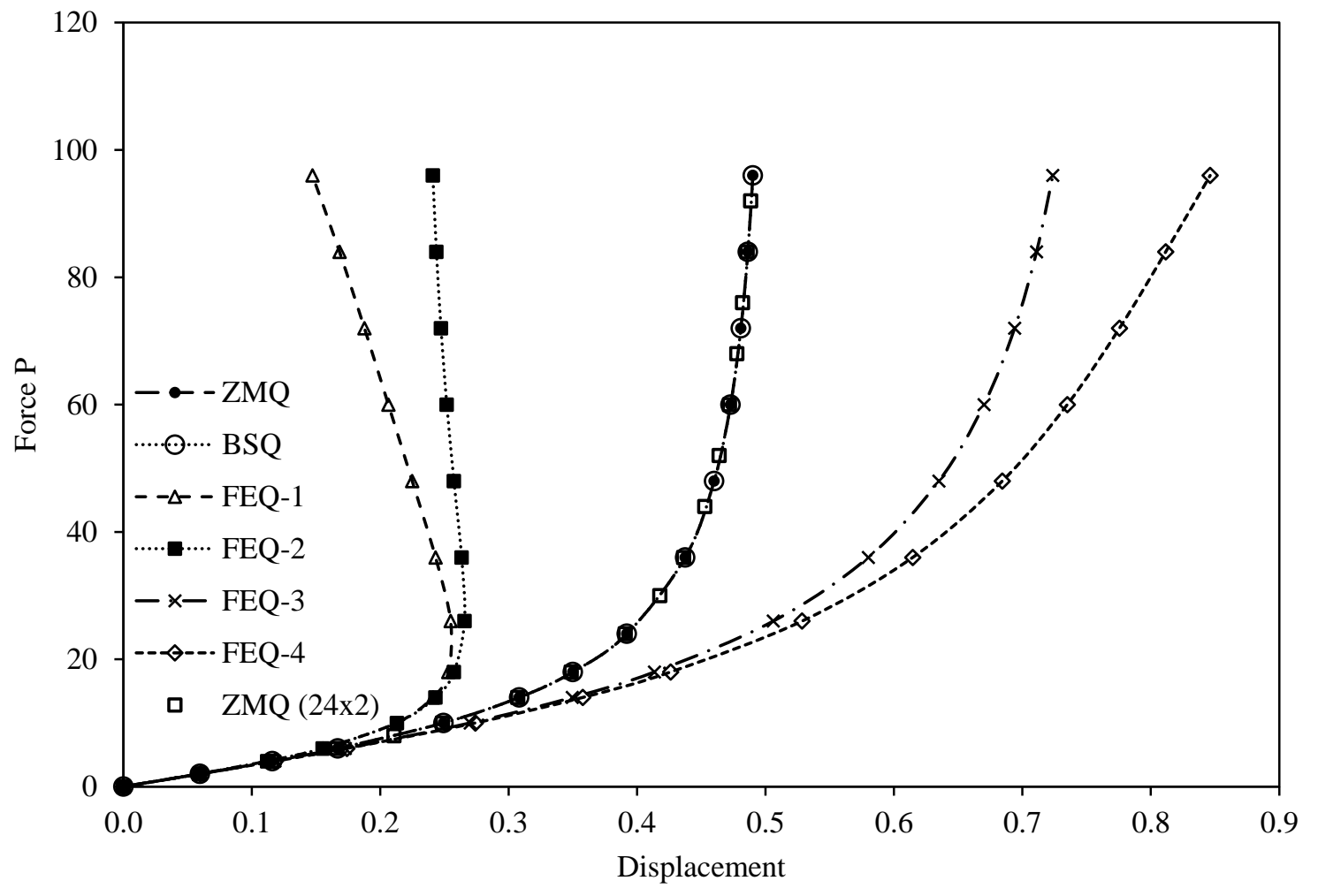

Figure 13: Load-displacement curves for $12 \times 1$ mesh of quadrilateral elements. 


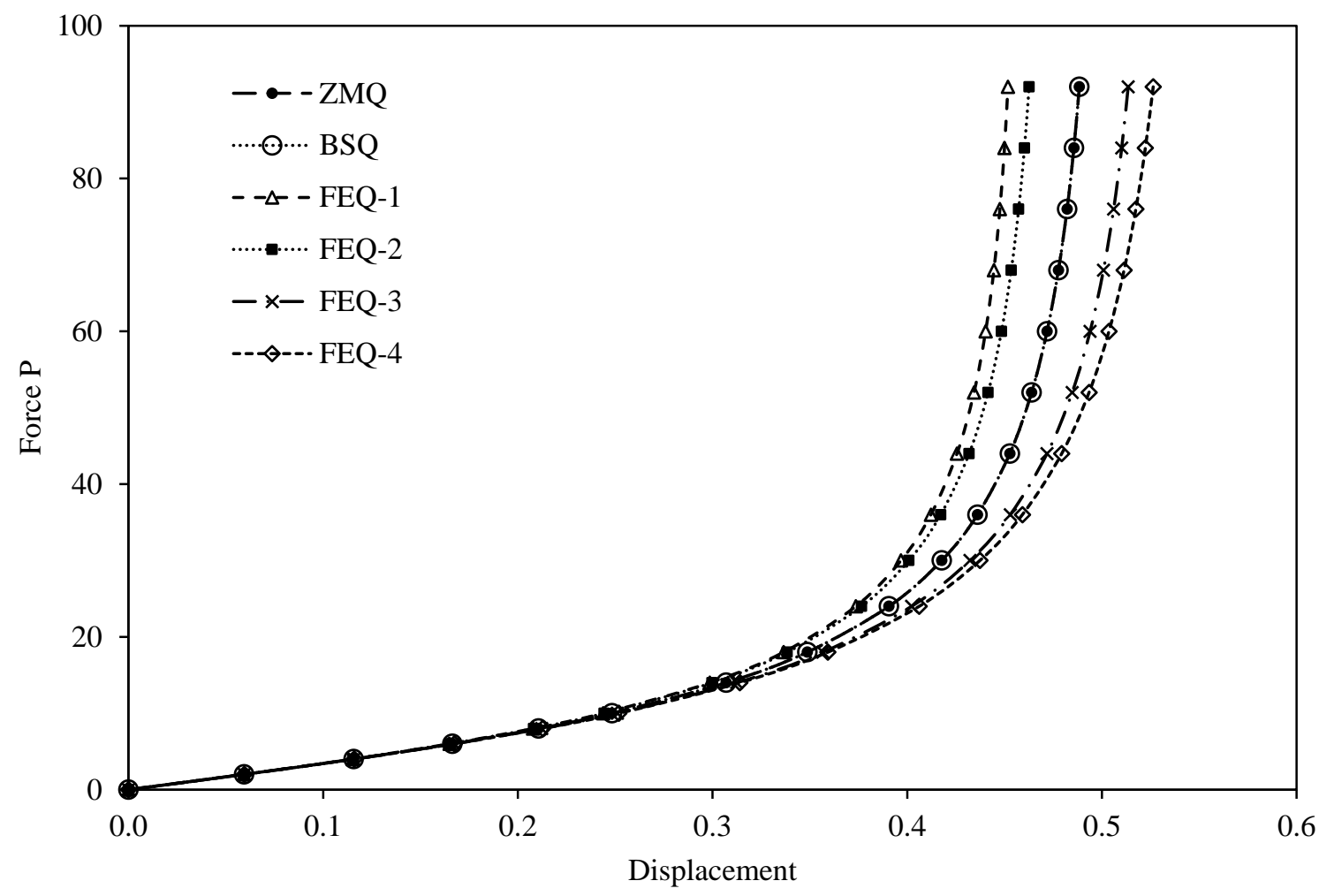

Figure 14: Load-displacement curves for $24 \times 2$ mesh of quadrilateral elements. 


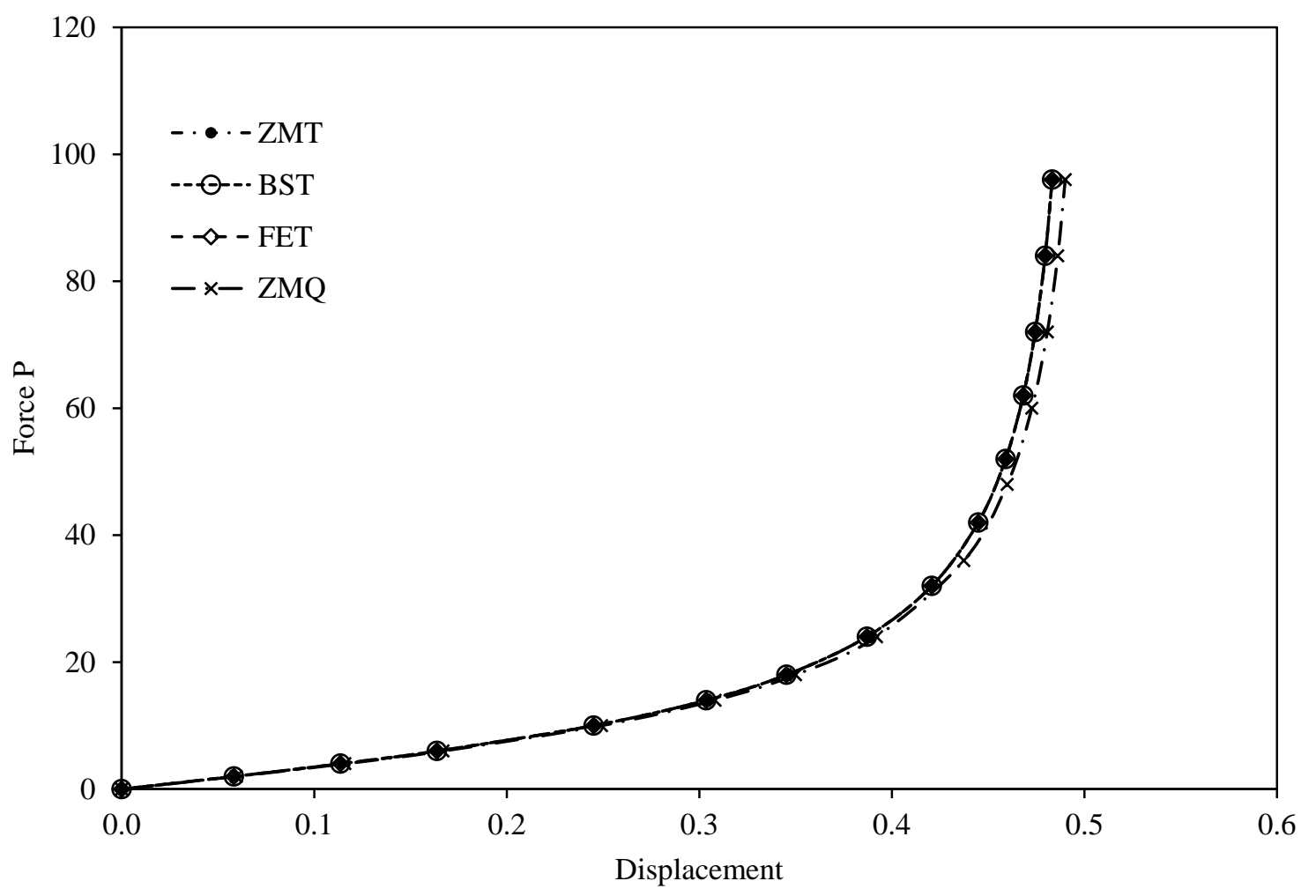

Figure 15: Load-displacement curves for $12 \times 1$ mesh of triangular elements. 

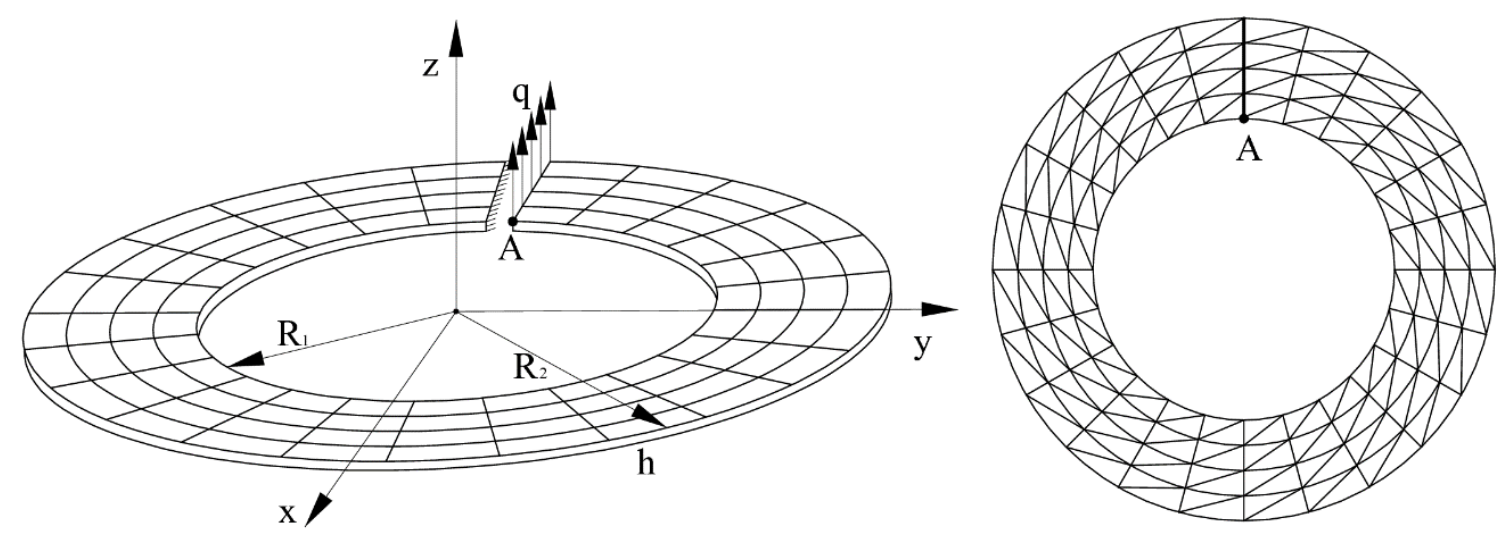

$\underline{24 \times 4}$ Triangular mesh

Figure 16: Annular plate subjected to end transverse distributed load. 


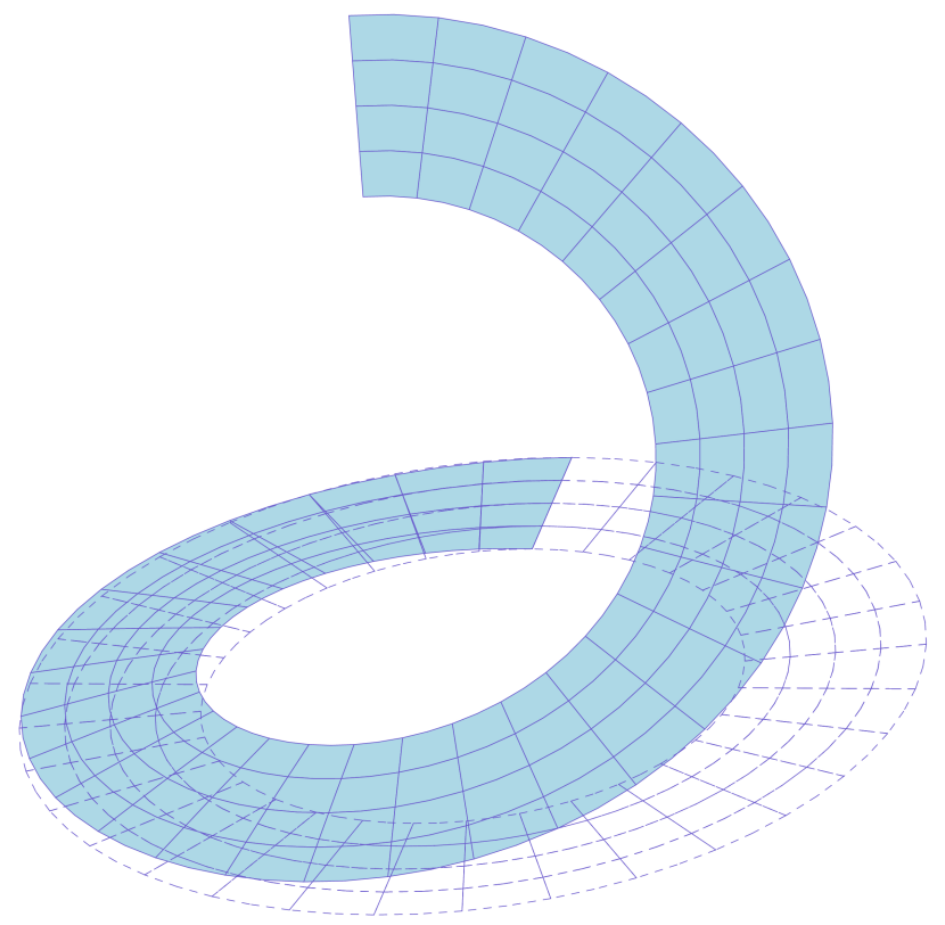

Figure 17: Deflected configuration of the annular plate with a $24 \times 4$ mesh of quadrilateral elements. 


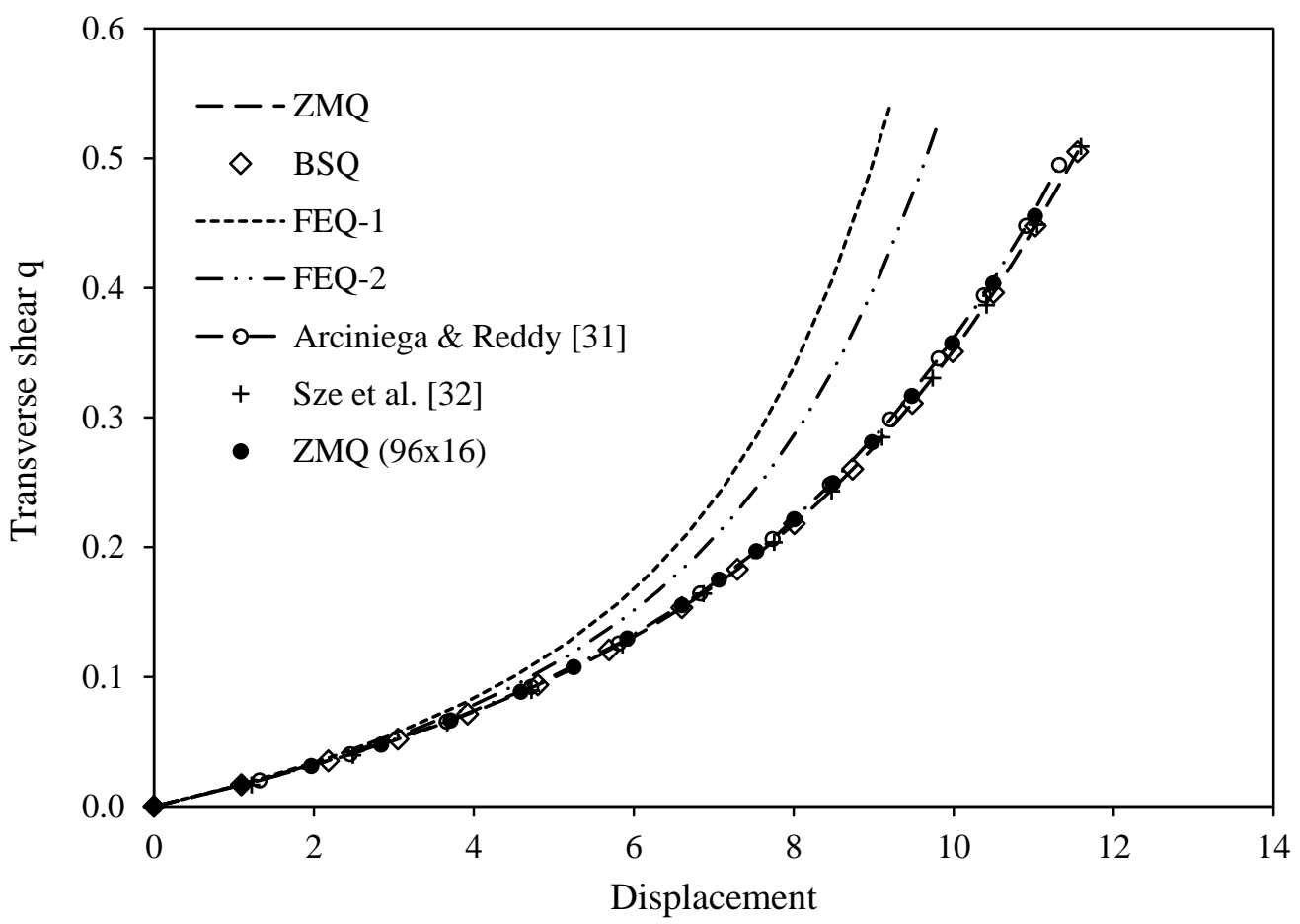

Figure 18: Load-displacement curves for $24 \times 4$ mesh of quadrilateral elements. 


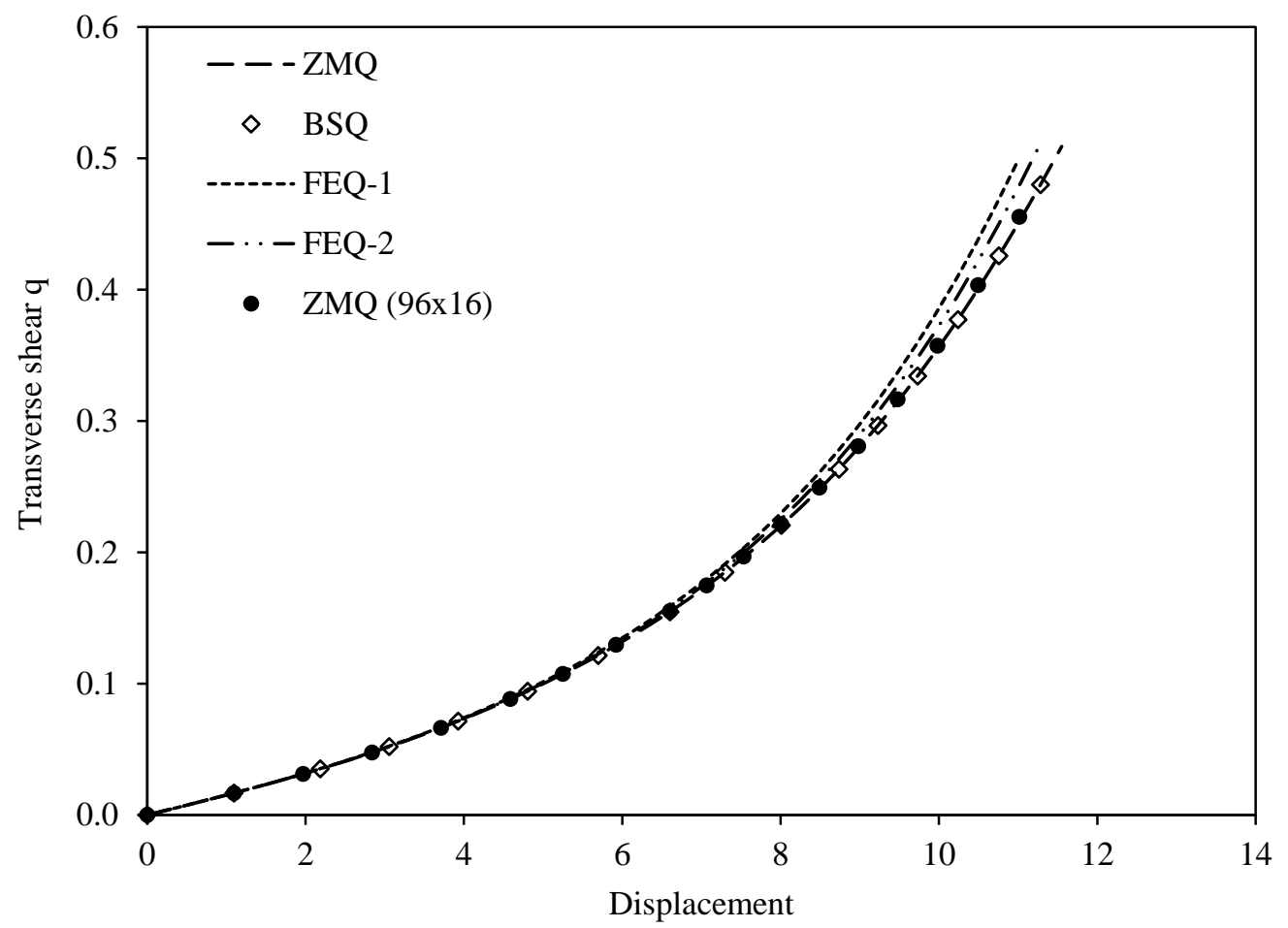

Figure 19: Load-displacement curves for $48 \times 8$ mesh of quadrilateral elements. 


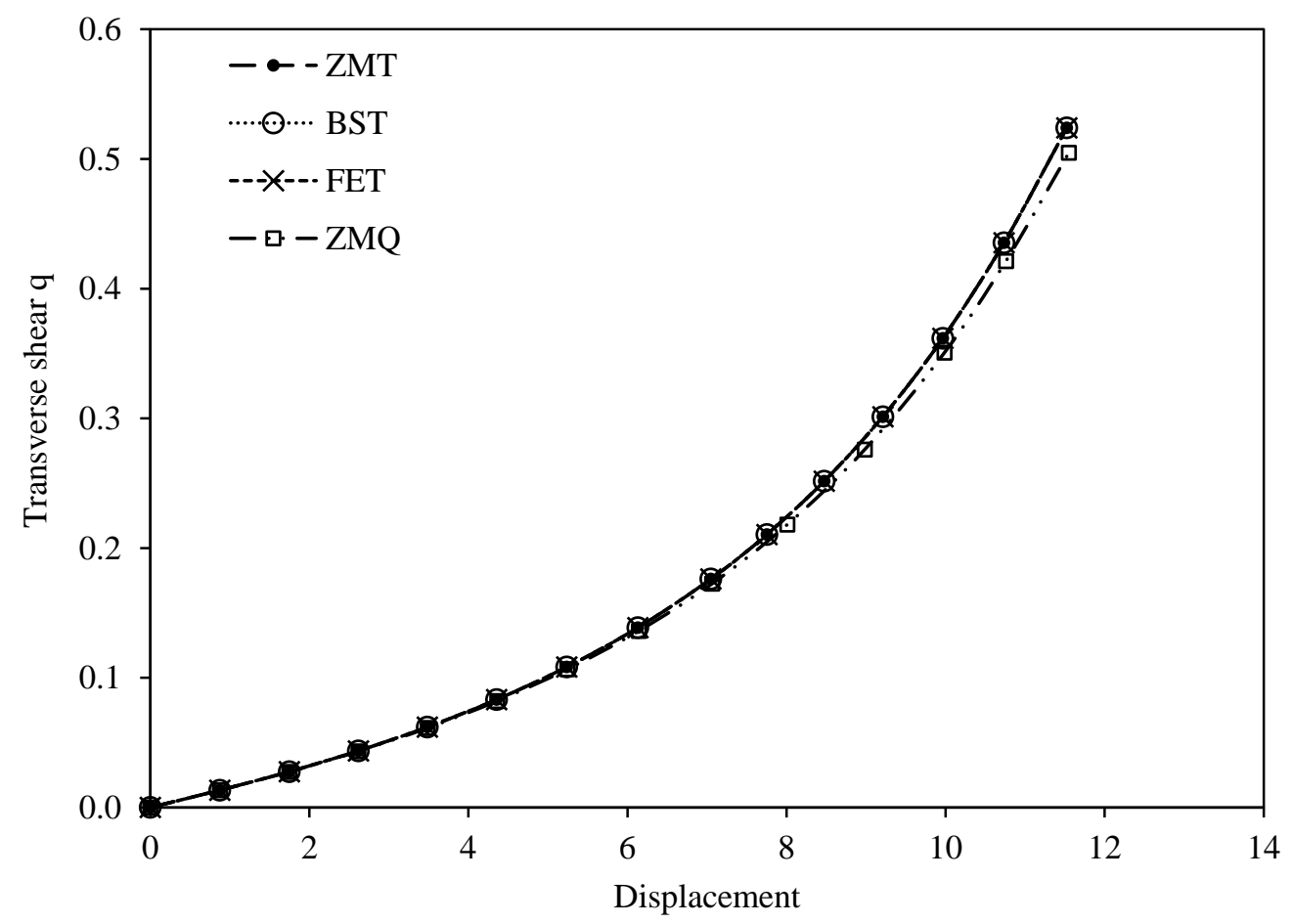

Figure 20: Load-displacement curves for $24 \times 4$ mesh of triangular elements. 


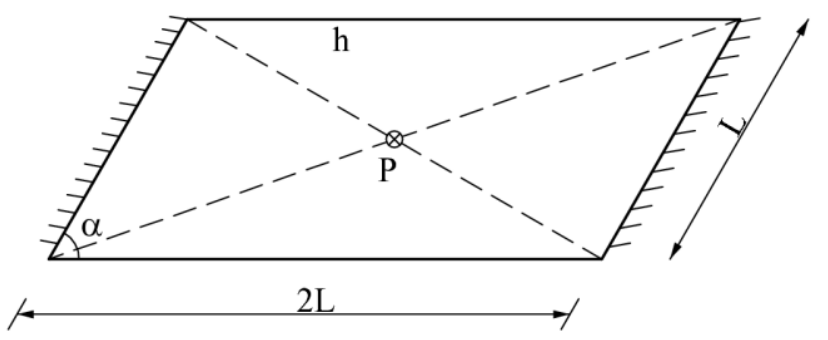

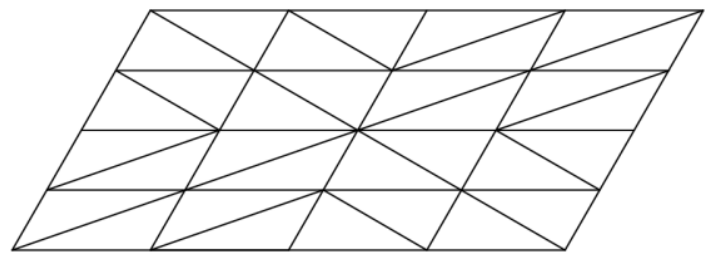

Triangular mesh pattern

Figure 21: $60^{\circ}$ skew plate subjected to central point load. 


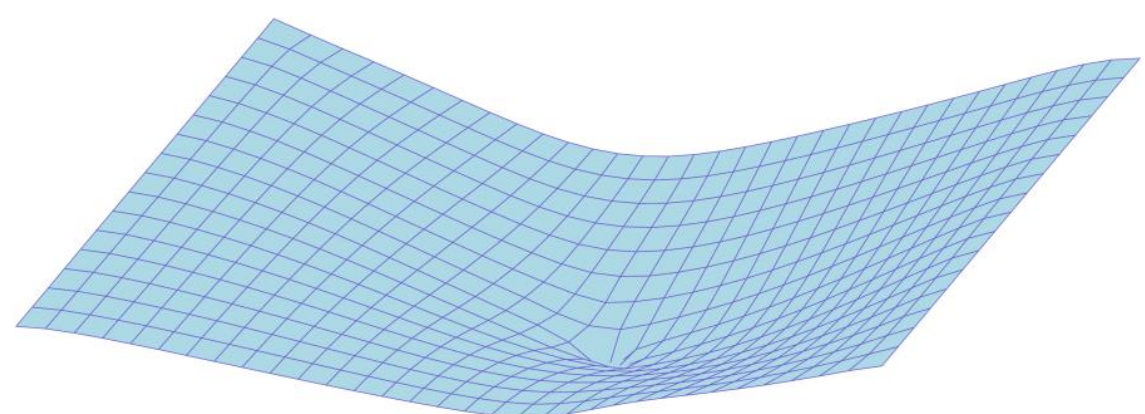

Figure 22: Deflected shape of the skew plate. 


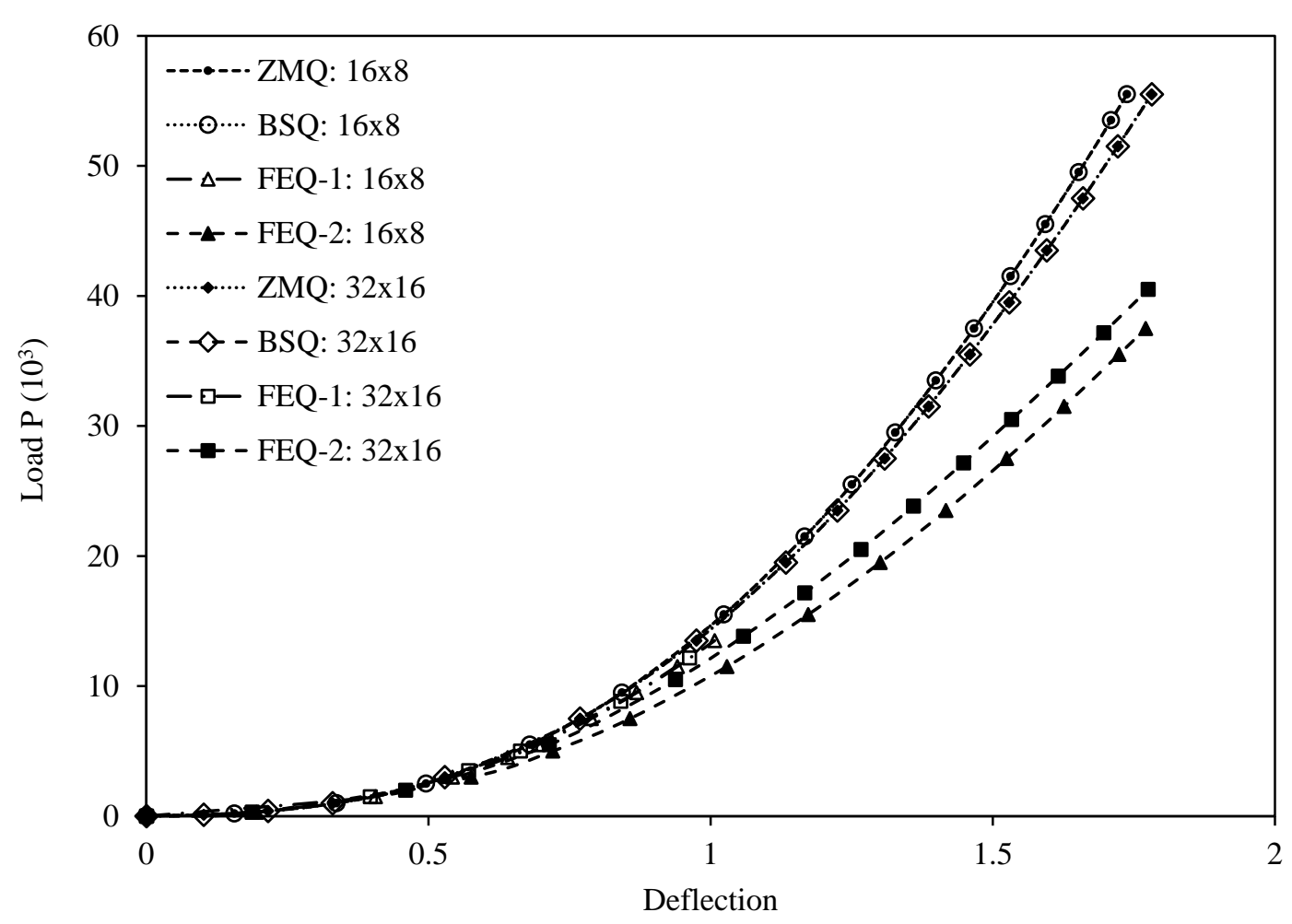

Figure 23: Load-displacement curves for $16 \times 8$ and $32 \times 16$ meshes of quadrilateral elements. 


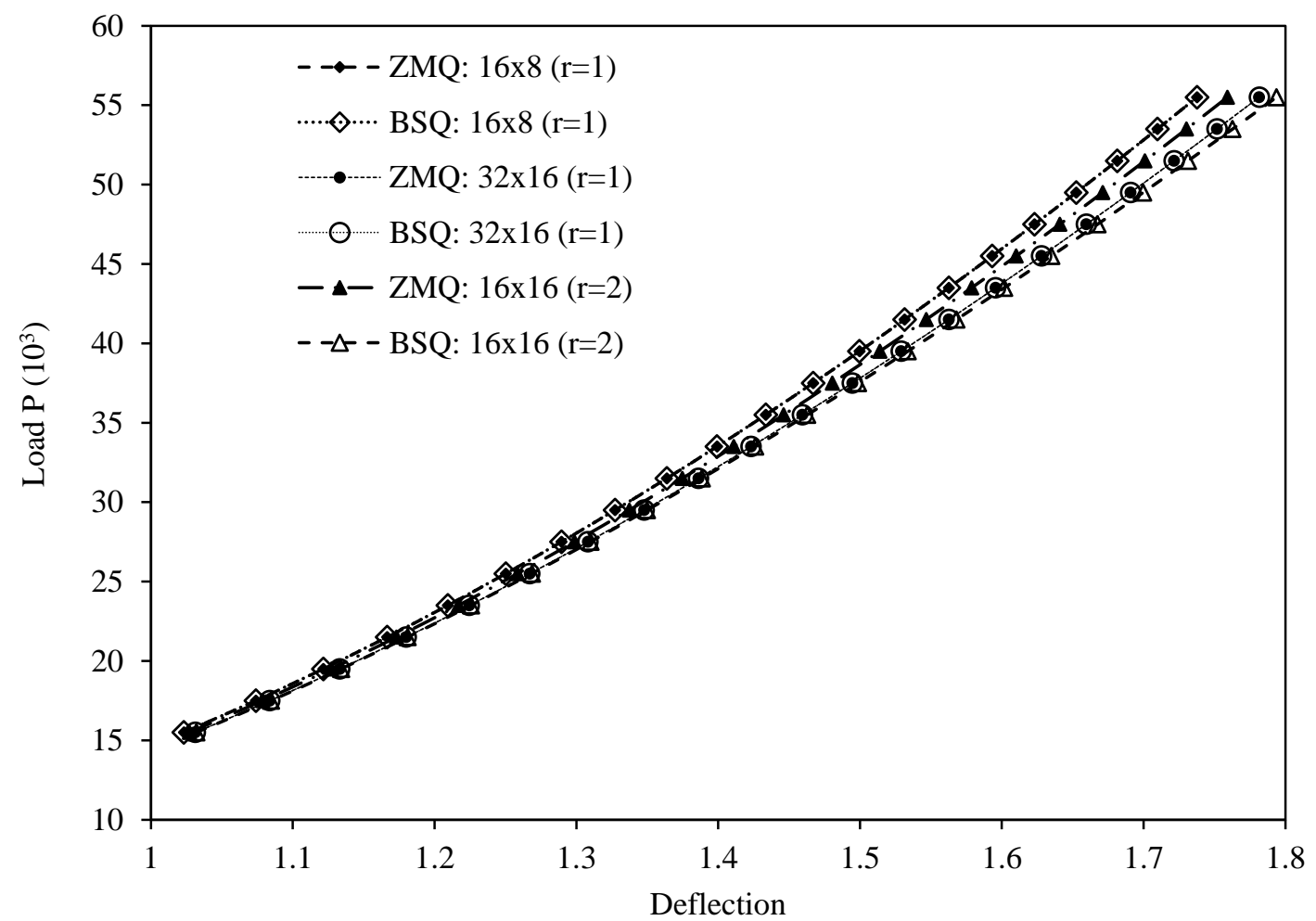

Figure 24: Influence of element aspect ratio $(\mathrm{r}=2)$ on predictions of bisector and zero-macrospin approaches. 


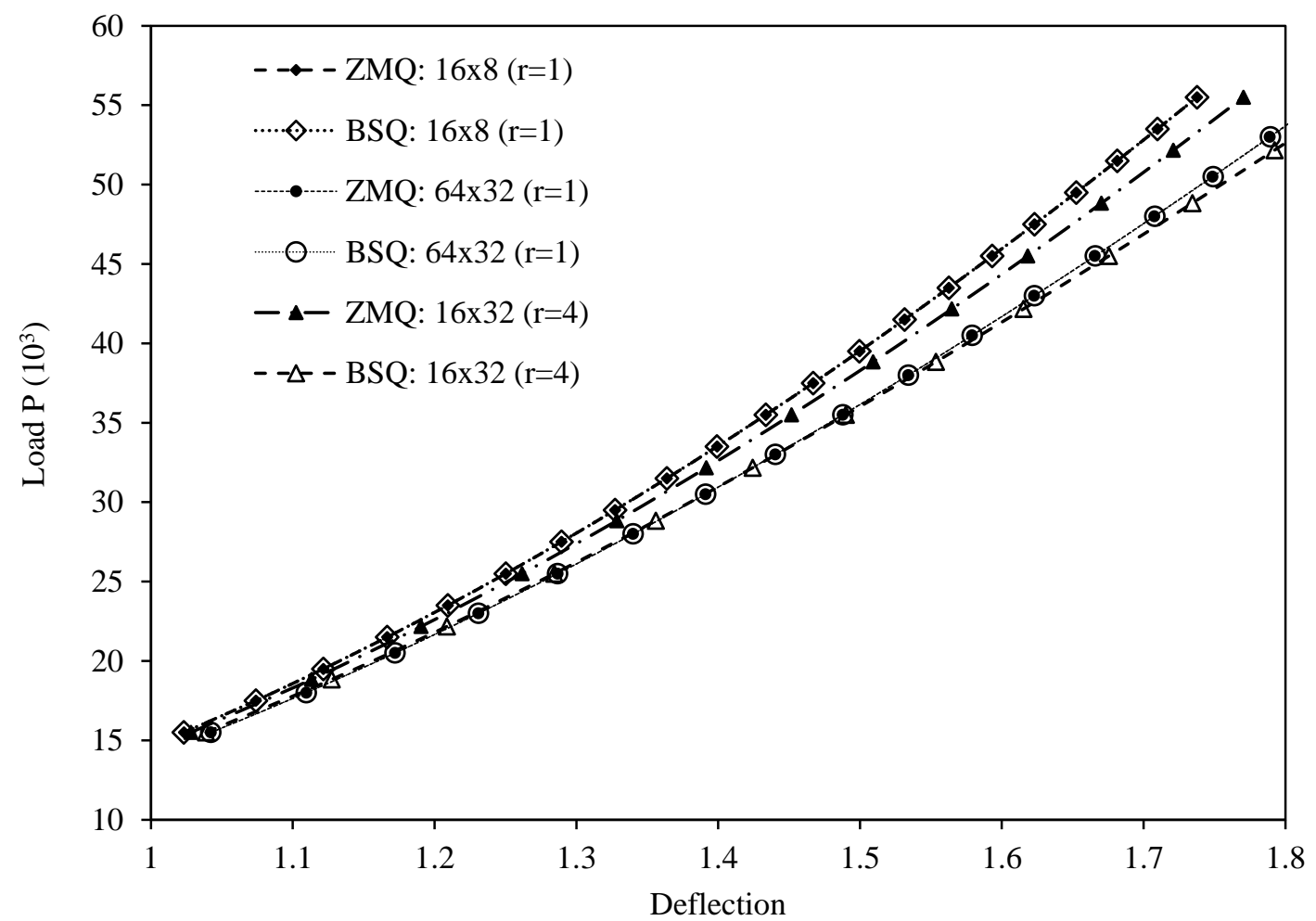

Figure 25: Influence of element aspect ratio $(\mathrm{r}=4)$ on predictions of bisector and zero-macrospin approaches. 


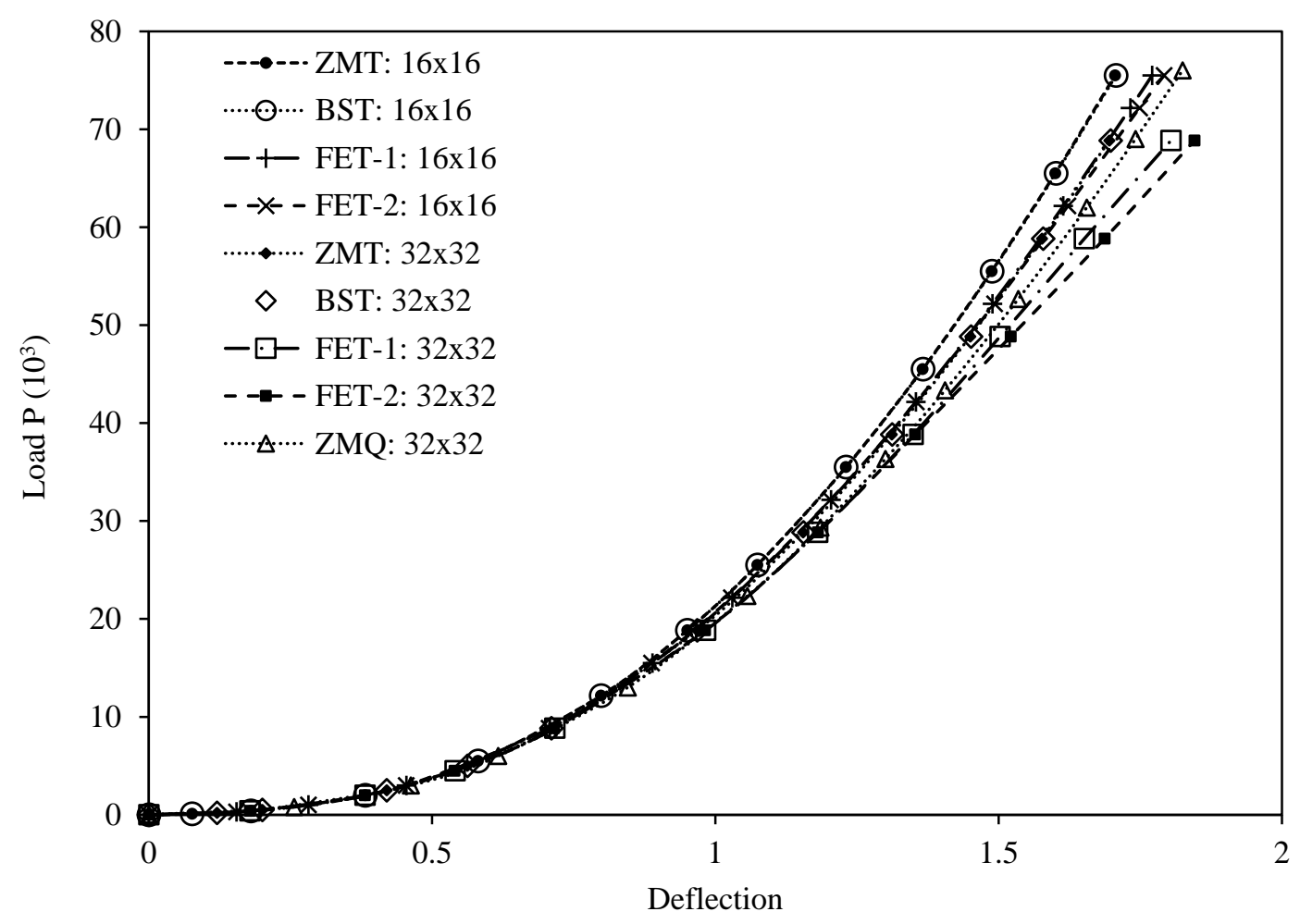

Figure 26: Load-displacement curves for $16 \times 16$ and $32 \times 32$ meshes of triangular elements. 


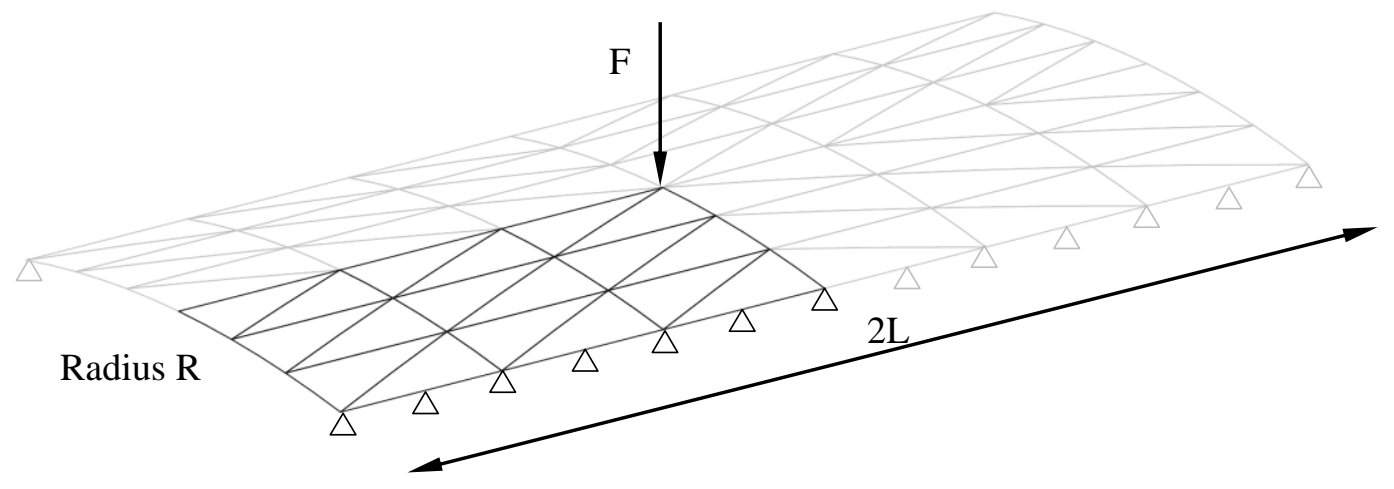

Figure 27: Hinged cylindrical shell under point load. 


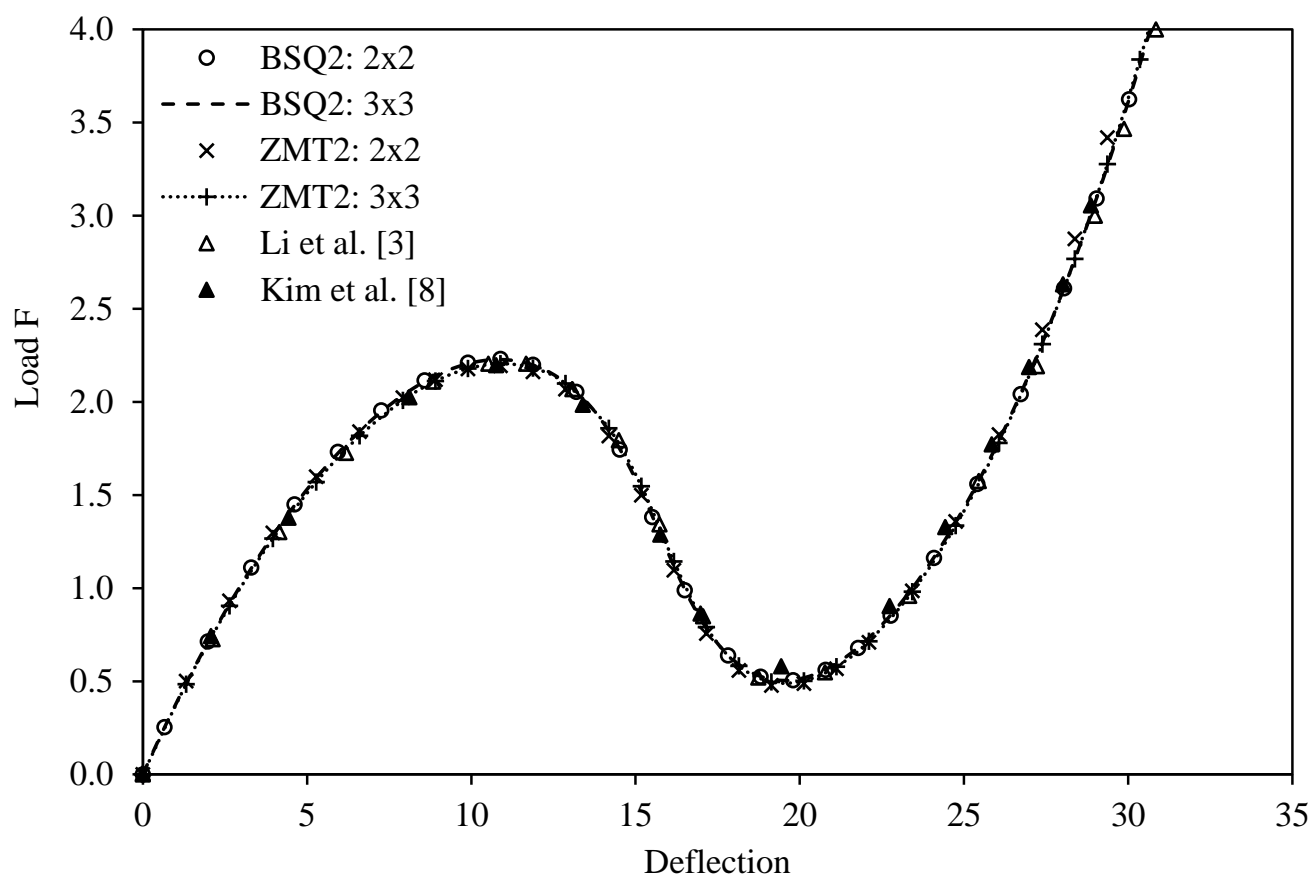

Figure 28: Load-deflection curves for alternative meshes of quadrilateral and triangular elements $(t=12.7)$. 


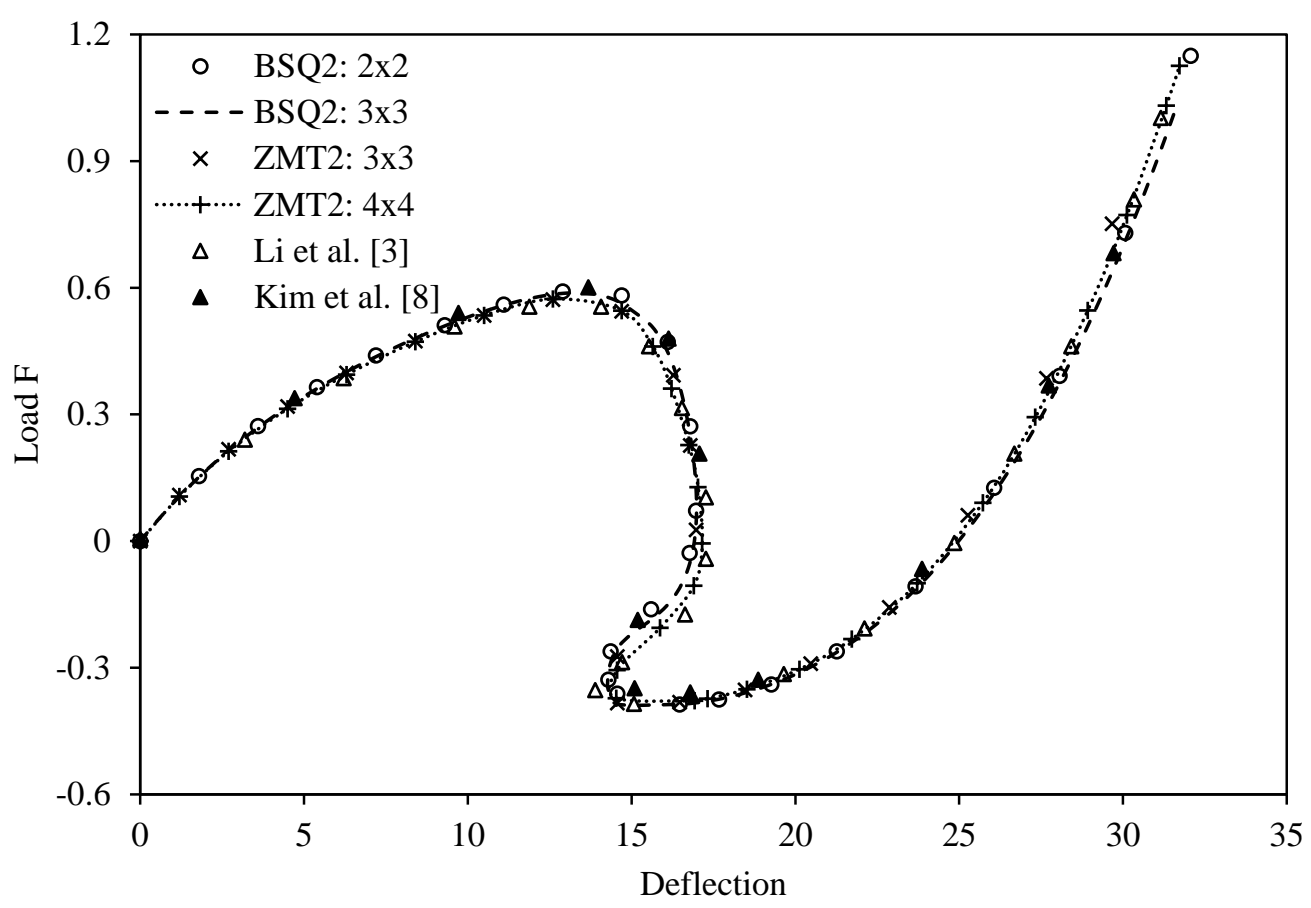

Figure 29: Load-deflection curves for alternative meshes of quadrilateral and triangular elements $(\mathrm{t}=6.35)$. 


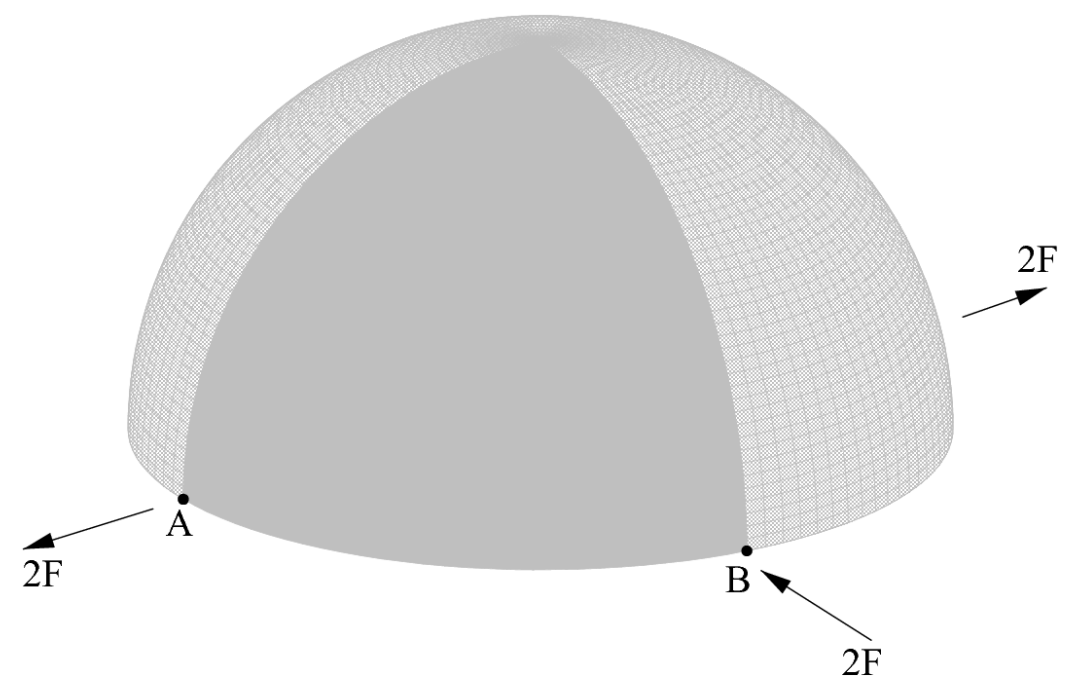

Figure 30: Hemispherical shell subjected to pinching forces. 


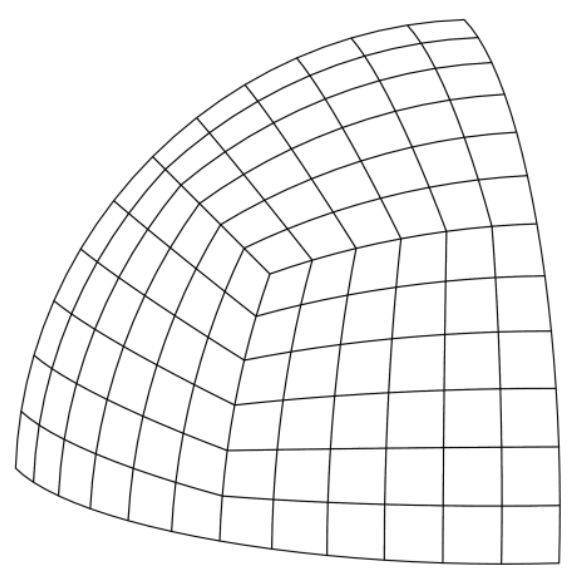

a. Quadrilateral element mesh

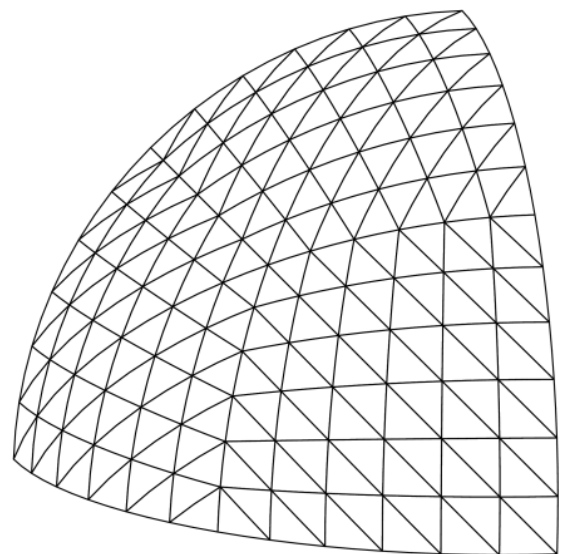

b. Triangular element mesh

Figure 31: Mesh patterns for a $6 \times 6$ mesh of elements. 


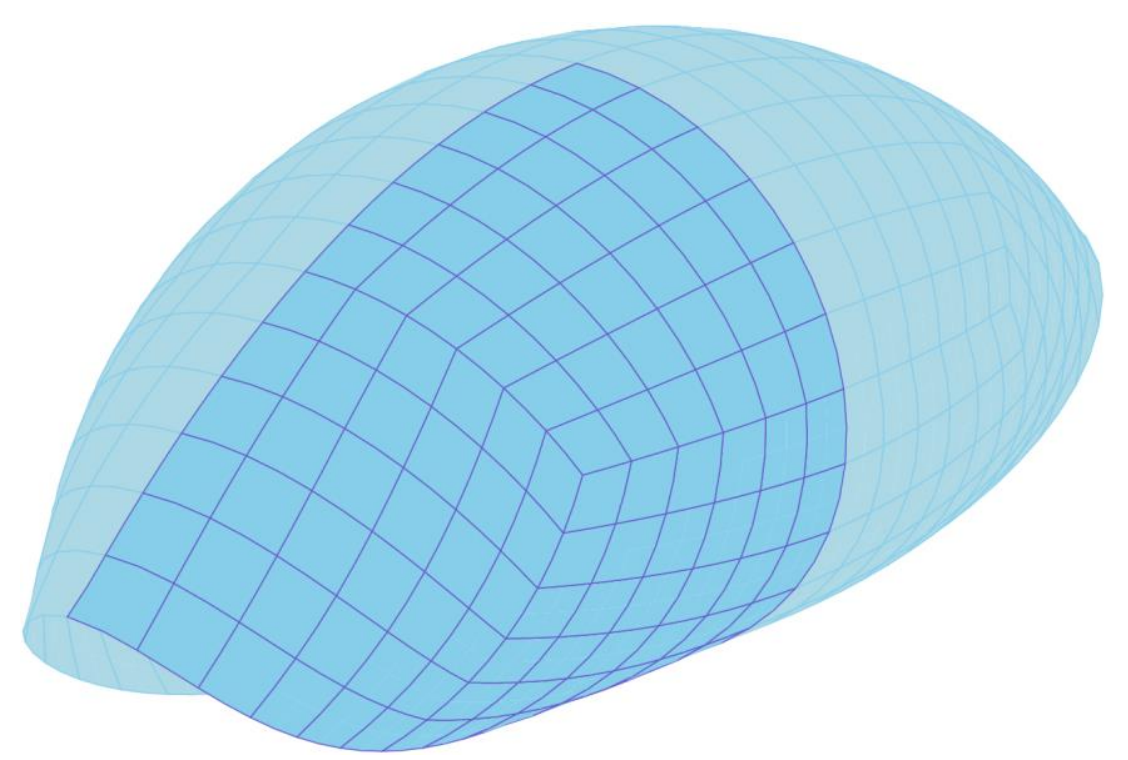

Figure 32: Deformed shape of the hemispherical shell. 


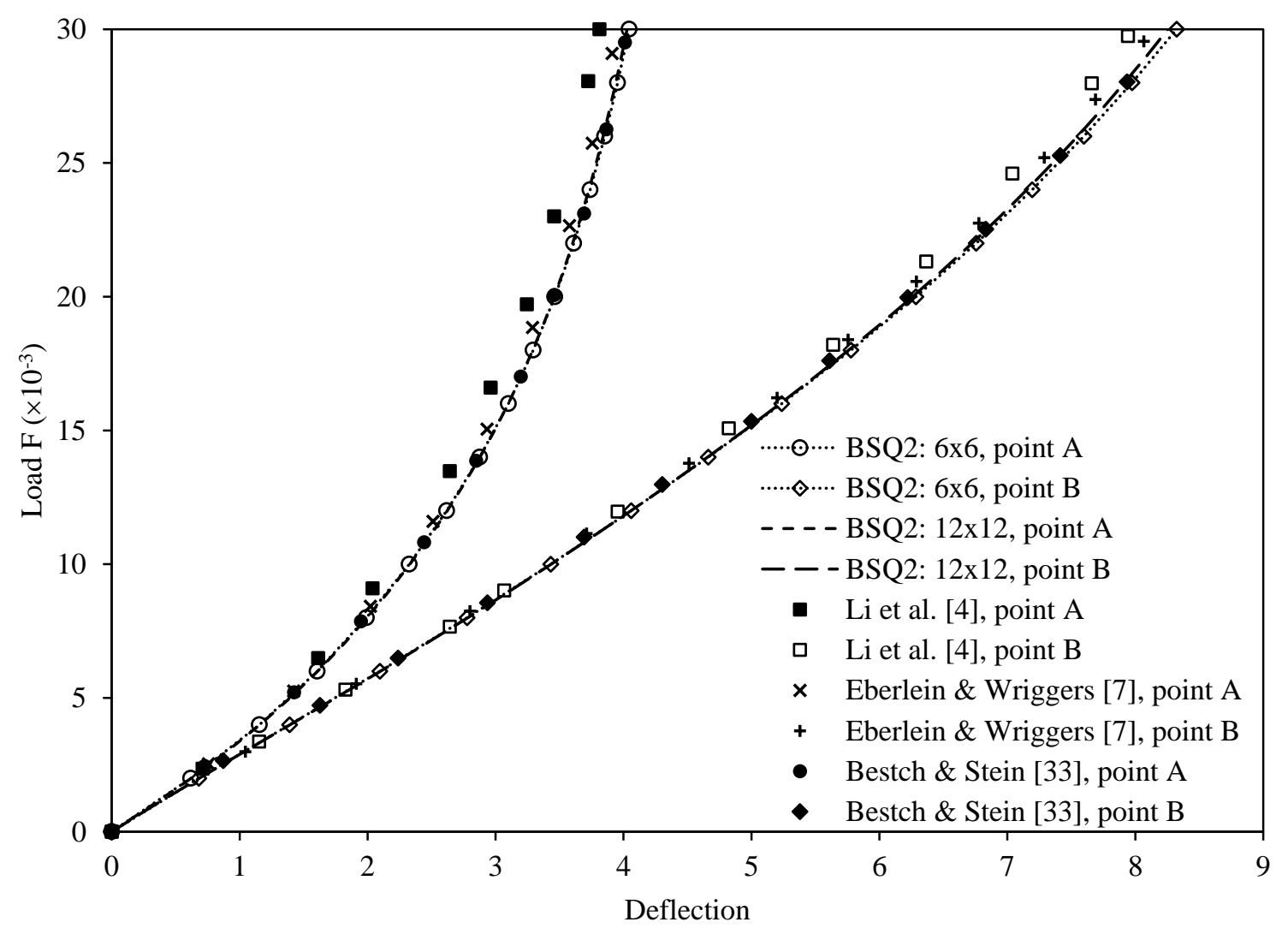

Figure 33: Load-deflection curves at point A and B for $6 \times 6$ and $12 \times 12$ meshes of quadrilateral elements. 


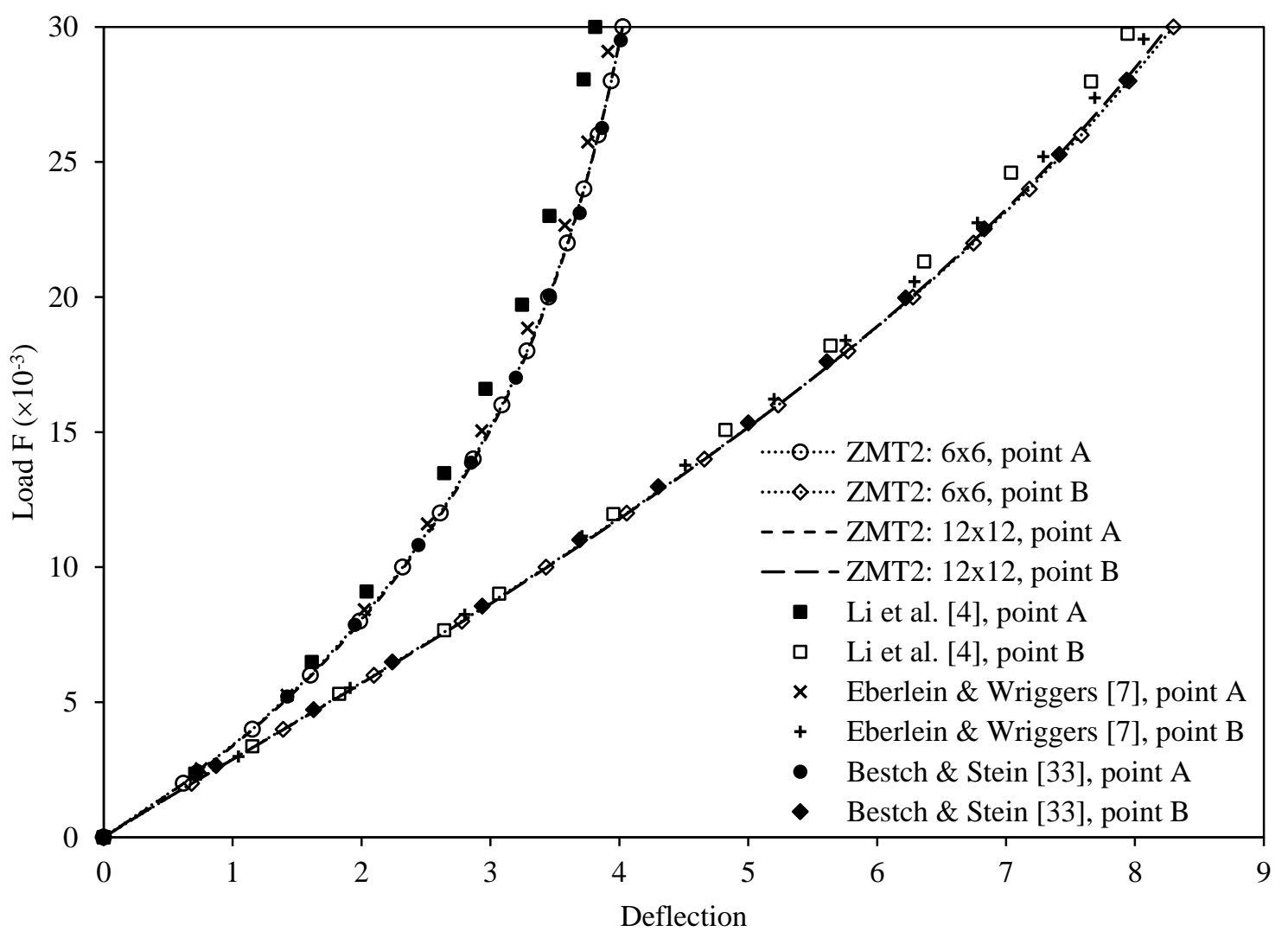

Figure 34: Load-deflection curves at point A and B for $6 \times 6$ and $12 \times 12$ meshes of triangular elements. 

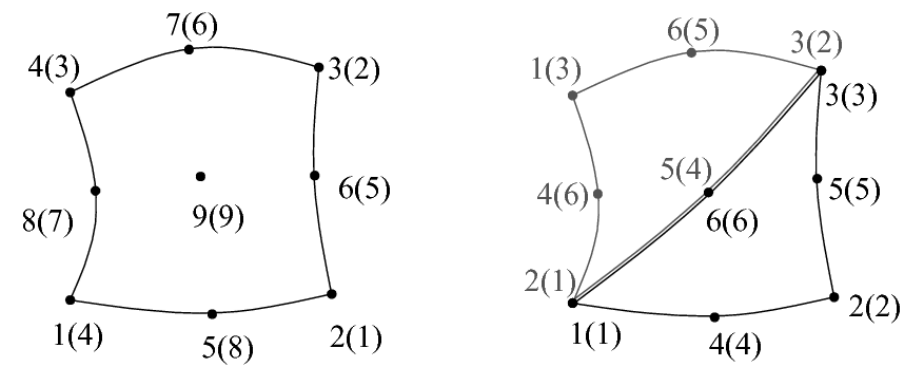

Figure 35: Alternative nodal ordering schemes for 9-noded and 6-noded elements. 